\title{
Development of a Misaligned Tropical Cyclone
}

\author{
DAVID A. SCHECTER \\ NorthWest Research Associates, Boulder, Colorado \\ Konstantinos MENELAOU \\ Department of Atmospheric and Oceanic Sciences, McGill University, Montreal, Quebec, Canada
}

(Manuscript received 21 March 2019, in final form 11 September 2019)

\begin{abstract}
A cloud-resolving model is used to examine the virtually shear-free evolution of incipient tropical cyclones initialized with different degrees of misalignment between the lower- and middle-tropospheric centers of rotation. Increasing the initial displacement of rotational centers (the tilt) from a negligible value to several hundred kilometers extends the time scale of hurricane formation from 1 to 10 days. Hindered amplification of the maximum tangential velocity $v_{m}$ at the surface of a strongly perturbed system is related to an extended duration of misalignment resulting from incomplete early decay and subsequent transient growth of the tilt magnitude. The prolonged misalignment coincides with a prolonged period of asymmetric convection peaked far from the surface center of the vortex. A Sawyer-Eliassen model is used to analyze the disparity between azimuthal velocity tendencies of selected pre-tropical storm vortices with low and high degrees of misalignment. Although no single factor completely explains the difference of intensification rates, greater misalignment is linked to weaker positive azimuthal velocity forcing near $v_{m}$ by the component of the mean secondary circulation attributed to heating by microphysical cloud processes. Of note regarding the dynamics, enhanced tilt only modestly affects the growth rate of kinetic energy outside the core of the surface vortex while severely hindering intensification of $v_{m}$ within the core for at least several days. The processes controlling the evolution of the misalignment associated with inefficient development are examined in detail for a selected simulation. It is found that adiabatic mechanisms are capable of driving the transient amplification of tilt, whereas diabatic processes are essential to ultimate alignment of the tropical cyclone.
\end{abstract}

\section{Introduction}

Decades of observational studies have provided convincing evidence that sufficiently intense deep-layer vertical wind shear in the environment will hinder the development ${ }^{1}$ of a tropical cyclone (Gray 1968; McBride and Zehr 1981; DeMaria et al. 2001; Kaplan et al. 2010; Tang and Emanuel 2012). Complementary modeling studies have largely corroborated this empirical finding and have substantially advanced our knowledge of the underlying dynamics (e.g., Tory et al. 2007; Rappin and Nolan 2012; Tao and Zhang 2014). The precise quantitative impact of deep-layer shear has been shown to depend on details of the associated height-dependent

\footnotetext{
${ }^{1}$ In this paper, development refers to formation and prehurricane intensification.

Corresponding author: David A. Schecter, schecter@nwra.com
}

wind profile, the surrounding distribution of moisture, and the sea surface temperature (ibid., Ge et al. 2013; Finocchio et al. 2016; Onderlinde and Nolan 2016). It is possible that circumstances exist under which weak-tomoderate shear can assist early development (Molinari et al. 2004; Davis and Bosart 2006; Musgrave et al. 2008; Nolan and McGauley 2012). However, it is more commonly inferred from modeling results that vortex misalignment (tilt) induced by shear plays an important role in frustrating the emergence of nearly saturated air and the generation of a robust symmetric component of convection over the central region of the lowertropospheric circulation, which would otherwise expedite surface spinup.

A number of the aforementioned modeling studies have examined idealized scenarios in which an immature tropical cyclone on the $f$ plane is exposed to a constant ambient shear flow of moderate amplitude. In this paradigm, a tilt develops pointing downshear and precesses toward an upshear orientation. Upon starting to tilt 
upshear, the vortex begins to realign. One might imagine that the decay of upshear tilt is primarily driven by differential advection, as in analogous simulations of certain dry adiabatic vortices that are otherwise unresilient (e.g., Fig. 8 of Reasor et al. 2004). Rios-Berrios et al. (2018) suggest that a more complex alignment mechanism involving both diabatic and adiabatic processes is more probable. In a general sense, their assessment seems consistent with some of the more realistically configured simulations of sheared tropical storm development, in which the convective generation and subsequent domination of a deep subvortex within the misaligned parent cyclone can be essential to the pertinent alignment process (Nguyen and Molinari 2015; cf. Chen et al. 2018). Regardless of the particular mechanism, the realignment of a tilted tropical cyclone during its development often appears to be a catalyst for the intensification of maximal winds.

The preceding discussion suggests that an integral part of understanding development is understanding alignment. As a first step toward improving current understanding of intrinsic vortex alignment mechanisms, it seems reasonable to remove environmental wind shear from the problem. Such removal eliminates the possibility that alignment is linked to precession of tilt into an upshear orientation. The remaining dynamics is nevertheless rich, and in need of further elucidation. Earlier studies of shear-free alignment have tended to focus on essentially adiabatic mechanisms found in simplified models where moisture effects are represented merely by the effects of reducing static stability (Polvani 1991; Viera 1995; Reasor and Montgomery 2001; Schecter and Montgomery 2003,2007). Expanding this work to the realm of cloud-resolving models is necessary to account for fundamentally distinct diabatic pathways of alignment that may occur with a more realistic treatment of moist convection. The potentially novel pathways revealed by an expanded study could very well have direct relevance to conceivable situations in nature where a substantial tilt of the vortex exists under fairly weak background shear [see, e.g., sections $4 a-4 b$ of Davis and Ahijevych (2012)]. The conditions under which such a system might rapidly transition to quasi-symmetric intensification or linger in a detrimental state of misalignment are largely unknown.

The general purpose of this paper is to begin a methodical study of the nature of shear-free development (alignment and intensification) in cloud-resolving numerical simulations of tropical cyclones with variable degrees of initial tilt. Intensification of both the maximum wind speed and kinetic energy of the primary surface circulation will be examined. An effort will be made to elucidate the variation of moist convection with tilt magnitude and the consequences on the angular momentum budget of the basic state of the vortex. Conclusions regarding the effects of tilt on the organization of convection and the temporal amplification of maximum wind speed will not stray far from those found in previous studies of developing tropical cyclones exposed to moderate deep-layer shear. On the other hand, our simulated vortices will follow somewhat distinct pathways toward the state of alignment that facilitates intensification. For example, the tilt of a strongly perturbed tropical cyclone will be shown to undergo an extended intermediate growth phase (following earlier decay) that cannot be attributed to forcing by domain-averaged shear. The importance of adiabatic and diabatic mechanisms in this and other stages of tilt evolution will be addressed.

The remainder of this paper is organized as follows. Section 2 describes our numerical experiments and our method for measuring the misalignment of a developing system. Section 3 examines the impact of misalignment on tropical cyclone development, and section 4 examines the tilt dynamics primarily in the context of an illustrative example. Section 5 recapitulates our findings. The appendixes provide supplemental details and explain a number of techniques used to analyze our simulations.

\section{Basic methodology}

The forthcoming discussions of our methodology and results require a preliminary remark on notation. In this paper, the properties of a tropical cyclone are usually described in a cylindrical coordinate system. The coordinates $r, \varphi$, and $z$ respectively denote radius, azimuth, and height above sea level. The variables $u, v$, and $w$ respectively represent the radial, azimuthal, and vertical velocity fields. An arbitrary fluid variable is sometimes decomposed into an azimuthal mean dressed with an overbar, and a perturbation dressed with a prime. For example, the azimuthal velocity field is given by $v=\bar{v}(r, z, t)+v^{\prime}(r, \varphi, z, t)$, in which $t$ is time.

\section{a. Numerical simulations}

The numerical simulations are conducted with Cloud Model 1 (CM1-r19.5; Bryan and Fritsch 2002) in an energy-conserving mode of operation. Unless stated otherwise, the model is configured with a variant of the two-moment Morrison microphysics parameterization (Morrison et al. 2005, 2009), having graupel as the large icy-hydrometeor category and a constant cloud-droplet concentration of $100 \mathrm{~cm}^{-3}$. The influence of subgrid turbulence above the surface is accounted for by an anisotropic Smagorinsky-type closure. The nominal mixing lengths are tailored for tropical cyclones on grids that are deemed insufficiently dense for a standard large-eddysimulation scheme. The horizontal mixing length increases 
from 0.1 to $0.7 \mathrm{~km}$ as the underlying surface pressure decreases from 1015 to $900 \mathrm{hPa}$. The vertical mixing length increases asymptotically to $50 \mathrm{~m}$ with increasing $z$. Surface fluxes are parameterized with bulk-aerodynamic formulas. The momentum exchange coefficient $C_{d}$ increases with the surface wind speed from $10^{-3}$ to 0.0024 above $25 \mathrm{~m} \mathrm{~s}^{-1}$ [compare with Fairall et al. (2003) and Donelan et al. (2004)]. The enthalpy exchange coefficient is given by $C_{e}=0.0012$ roughly based on the findings of Drennan et al. (2007). Heating associated with frictional dissipation is activated. The computationally expensive parameterization of radiative transfer is turned off under the provisional assumption that the associated diabatic forcing is unessential to understanding the slowdown of intensification induced by misalignments of incipient tropical cyclones.

The model is set up on a doubly periodic oceanic $f$ plane with a sea surface temperature of $28^{\circ} \mathrm{C}$ and a Coriolis parameter of $f=5 \times 10^{-5} \mathrm{~s}^{-1}$ corresponding to a latitude of $20^{\circ} \mathrm{N}$. The dynamical system is discretized on a stretched rectangular grid. The horizontal grid spans $2660 \mathrm{~km}$ in each orthogonal coordinate. The $800 \times 800 \mathrm{~km}^{2}$ central region of the horizontal mesh has grid increments of $1.25 \mathrm{~km}$. At the four corners of the horizontal domain, the grid increments are $13.75 \mathrm{~km}$. The vertical grid has 73 points and extends upward to $z=29.2 \mathrm{~km}$. The vertical grid spacing gradually increases from 50 to 400 to $750 \mathrm{~m}$ as $z$ increases from 0 to 8 to $29 \mathrm{~km}$. Rayleigh damping is imposed above $z=25 \mathrm{~km}$.

The control experiment is initialized with a predepression vortex loosely modeled after the findings of the PREDICT field campaign in the North Atlantic basin (Davis and Ahijevych 2012, 2013; Komaromi 2013; Smith and Montgomery 2012). The relative vertical vorticity distribution is confined to $z<10.5 \mathrm{~km}$ and $r<r_{b}=750 \mathrm{~km}$, where it is given by

$$
\begin{aligned}
\zeta= & \zeta_{o} \cos \left[\frac{\pi\left(z-z_{c}\right)}{2 h(z)}\right]\left\{\frac{r}{r_{1}}\left[1-e^{-\left(r / r_{1}\right)^{2}}\right]+e^{-\left(r / r_{2}\right)^{8}}\right\}^{-1} \\
& -\delta \zeta(z)
\end{aligned}
$$

in which $\zeta_{o}=2.4 \times 10^{-4} \mathrm{~s}^{-1}, z_{c}=3 \mathrm{~km}, r_{1}=70 \mathrm{~km}, r_{2}=$ $400 \mathrm{~km}$, and $h=7.5 \mathrm{~km}(5.6 \mathrm{~km})$ for $z>z_{c}\left(z<z_{c}\right)$. The correction $\delta \zeta$ guarantees zero net circulation and amounts to the areal average of the preceding term over a horizontal disc of radius $r_{b}$. The azimuthal velocity $v$ is obtained by inverting $\zeta$, whereas $u$ and $w$ are set to zero. The ambient $\left(r>r_{b}\right)$ pressure $p$ and virtual potential temperature $\theta_{v}$ are given by the Dunion (2011) moist tropical sounding (MTS); the perturbations to $p$ and $\theta_{v}$ linked to the vortex are iteratively adjusted to satisfy hydrostatic and gradient balance. The relative humidity with respect to liquid water (subscript $l$ ) is given by

$$
\mathrm{RH}_{l}=\mathrm{RH}_{0}+\left(\mathrm{RH}_{d}-\mathrm{RH}_{0}\right)\left[1-e^{-\left(r / r_{3}\right)^{2}}\right]
$$

Equation (2) imposes a moderate enhancement of middle-tropospheric relative humidity in the meso$\beta$-scale core of the vortex, which has been observed in developing systems shortly before genesis [see section $3 \mathrm{~b}$ of Davis and Ahijevych (2013)]. To elaborate, for $r$ appreciably greater than $r_{3}=325 \mathrm{~km}$, the relative humidity profile corresponds to that of the Dunion $\operatorname{MTS}\left[\mathrm{RH}_{d}(z)\right]$. As $r$ approaches 0 , the profile gradually increases to $\mathrm{RH}_{0}(z)$. The value of $\mathrm{RH}_{0}$ holds steady at 0.88 with increasing $z$ below $5.5 \mathrm{~km}$, whereupon it decays linearly up to $10 \mathrm{~km}$ and then matches $\mathrm{RH}_{d}$. Figure 1 depicts both the primary circulation and the moist thermodynamic structure of the initial condition.

The misalignment experiments are initialized with conditions derived from the control simulation $99 \mathrm{~h}$ into its development, shortly before one might declare the genesis of a warm core tropical cyclone. Figure 2 depicts the azimuthally averaged fluid variables at this time, henceforth labeled $t=0$. The absolute maximum of $\bar{v}$ occurring at $r=115.1 \mathrm{~km}$ and $z=3.7 \mathrm{~km}$ is $12.0 \mathrm{~m} \mathrm{~s}^{-1}$; the surface maximum is $8.6 \mathrm{~m} \mathrm{~s}^{-1}$ at $r=42.9 \mathrm{~km}$. An upper-tropospheric anticyclone (Fig. 2a) has emerged in the outflow of a weak secondary circulation (Fig. 2b). Furthermore, the relative humidity has broadly intensified throughout the central region of the vortex (Fig. 2c).

In each experiment, the misalignment is created using one of the following three techniques: impulsive separation (IS), impulsive separation plus damping (ISPD), and dry separation plus damping (DSPD). The IS method introduces forcing terms to the horizontal velocity equations that-in a frictionless quiescent atmosphere-would generate a transient shear flow in the Earth-stationary (ES) reference frame of the following form:

$$
\begin{aligned}
\mathbf{u}_{s}(z, t)= & \hat{\mathbf{x}} \frac{U_{s}}{2} \tanh \left(\frac{z-z_{l}}{\delta z_{l}}\right)\left[1.0+\tanh \left(\frac{z_{u}-z}{\delta z_{u}}\right)\right] \\
& \times\left\{\begin{array}{cl}
t / \delta \tau_{s}, & t<\delta \tau_{s} \\
1, & \delta \tau_{s} \leq t \leq \tau_{s} \\
1-\left(t-\tau_{s}\right) / \delta \tau_{s}, & \tau_{s}<t<\tau_{s}+\delta \tau_{s} \\
0, & t \geq \tau_{s}+\delta \tau_{s}
\end{array}\right.
\end{aligned}
$$



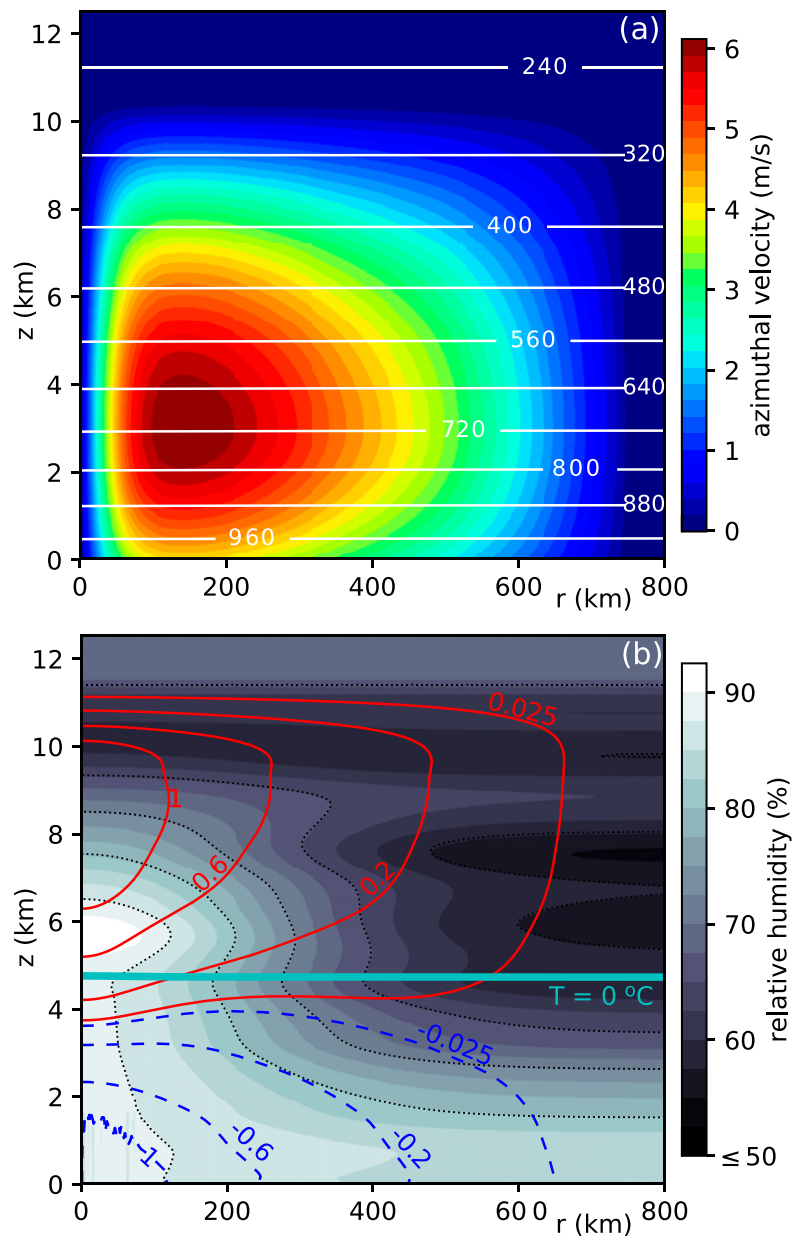

FIG. 1. Axisymmetric initial conditions for preliminary priming of the model. (a) Azimuthal velocity distribution (color) and pressure (contours; hPa). (b) Relative humidity with respect to ice $\left(T<0^{\circ} \mathrm{C}\right)$ or liquid $\left(T>0^{\circ} \mathrm{C}\right)$, and selected positive (solid red) and negative (dashed blue) isolines of the potential temperature $(\theta)$ anomaly, here defined by $\bar{\theta}-\bar{\theta}(r=800 \mathrm{~km}, z)$ and given in units of $\mathrm{K}$. The dotted black curves correspond to relative humidities of $57.5 \%, 65 \%, 72.5 \%, 80 \%$, and $87.5 \%$. The virtually horizontal cyan curve corresponds to the nominal freezing level, where the temperature $T$ is $0^{\circ} \mathrm{C}$.

in which $\hat{\mathbf{x}}$ is the unit vector pointing eastward, $\tau_{s}=5 \mathrm{~h}$, $\delta \tau_{s}=1 \mathrm{~h}, \delta z_{l}=0.5 \mathrm{~km}, z_{u}=21 \mathrm{~km}$, and $\delta z_{u}=1 \mathrm{~km}$. The adjustable parameters $U_{s}$ and $z_{l}$ respectively represent the maximum wind speed and the height at which easterly flow $\left(z<z_{l}\right)$ changes to westerly flow $\left(z>z_{l}\right)$. The Cartesian $x$ and $y$ components of the imposed velocity accelerations are respectively given by $F_{x}=\hat{\mathbf{x}} \cdot \partial \mathbf{u}_{s} / \partial t$ and $F_{y}=f \hat{\mathbf{x}} \cdot \mathbf{u}_{s}$. The ISPD method is equivalent to the IS method for $t \leq \tau_{s}+\delta \tau_{s}$, but adds a damping term of the form $-\langle\mathbf{u}\rangle_{x y} / \tau_{d}$ to the vector horizontal velocity equation over a finite time period of $4 \tau_{d}$ thereafter. Here, $\langle\mathbf{u}\rangle_{x y}$ is the horizontal domain average of the horizontal velocity vector $\mathbf{u}$ in the ES reference frame, and $\tau_{d}=1.5 \mathrm{~h}$. The point of the damping is to virtually remove the small amount of residual ambient shear that will have resulted from friction and the presence of the vortical system.

The DSPD method first removes all asymmetry in $\varphi$ from the vortex. Dissipative heating is eliminated, $C_{d}$ is reduced to $10^{-4}$, and $C_{e}$ is reduced to zero. The moisture parameterization is turned off and the water vapor is temporarily replaced with a passive tracer. The symmetric dry vortex is given one day to adjust toward a more balanced state, whereupon it is misaligned using variants of the ISPD method with adjustable values of $\tau_{s}$, $\delta \tau_{s}$ and $\tau_{d}$ that typically correspond to slower forcing. At the end of the forcing period, the passive tracer is replaced with water vapor. The mixing ratio is reduced where necessary to $99.5 \%$ of its saturation value with respect to ice/liquid at altitudes above/below the local freezing level to prevent the minor shock of instantaneous cloud formation. All physics parameterizations of the control experiment are restored, and time $t$ is reset to zero.

Table 1 lists all of the numerical experiments considered herein, except for the control run. The name of each experiment begins with the abbreviation of the misalignment method and ends with a code for two additional parameters. In this code, the symbol $X$ represents $2 U_{s} \tau_{s}$, and $\mathrm{Z}$ represents $z_{l}$; the rounded-down value (in kilometers) of each parameter is printed to the immediate right of the corresponding symbol. The value of $2 U_{s} \tau_{s}$ ranges from 100 to $400 \mathrm{~km}$, and can be viewed as a target magnitude for the tilt [Eq. (4)] that is initially forced on the vortex. The actual tilt magnitude moderately varies between IS/ISPD and DSPD simulations with the same parameter code. The actual tilt magnitude also depends to some degree on $z_{l}$, which equals $5.25,3.5$, or (in one case) $1.75 \mathrm{~km}$. It is found that the tilt dependence of tropical cyclone development in this study is basically consistent between groups of simulations with different values of $z_{l}$. Appendix A illustrates the diversity of vortex states within our simulation set immediately after the misalignments are created.

\section{b. Vortex center finding algorithm}

Many of the measurements made for this study depend on a reasonable technique for establishing the center of the vortex in a given layer of the atmosphere. The technique employed herein first obtains a massweighted vertical average of the relative vertical vorticity distribution $\zeta$ in a layer defined by $h_{-} \leq z \leq h_{+}$. The vorticity distribution on a regular fine grid (with spacing $l_{f}=1.25 \mathrm{~km}$ ) is then mapped onto a regular coarse grid (with spacing $l_{c}=23.75 \mathrm{~km}$ ). The mapping 

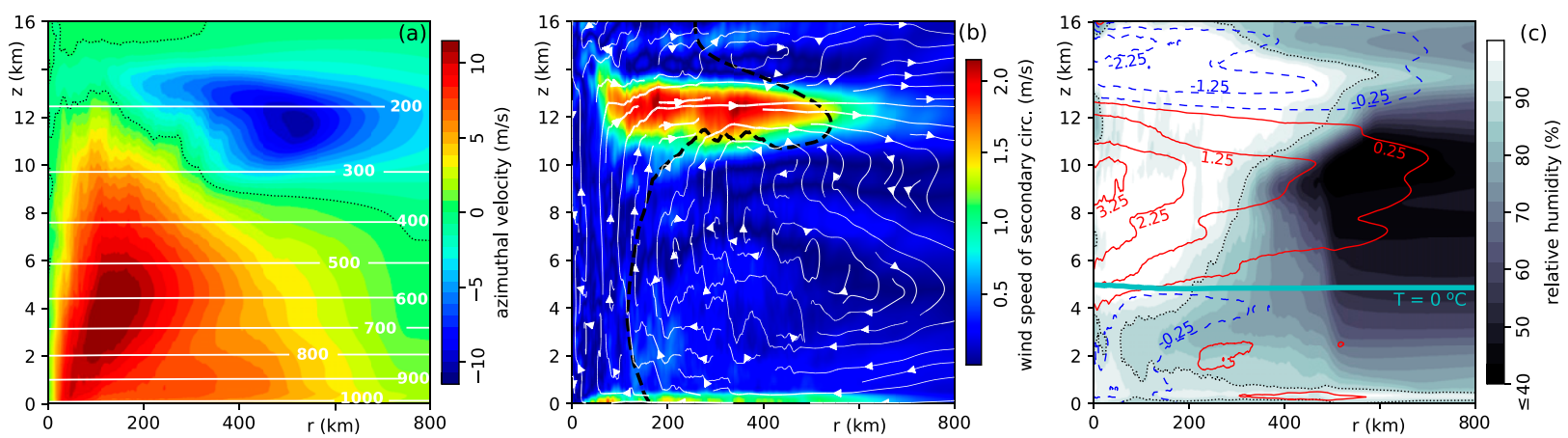

FIG. 2. Azimuthally averaged fields associated with the tropical disturbance after $99 \mathrm{~h}$ of priming and immediately before misalignment, when $t$ is reset to zero. (a) Azimuthal velocity $\bar{v}$ (color) and pressure $\bar{p}$ (white contours; hPa). The dotted black curves show where $\bar{v}=0$. (b) Streamlines (white) and magnitude (color) of the secondary velocity field $(\bar{u}, \bar{w})$. The dashed black reference contour is the isoline of angular momentum $\left(\bar{v} r+f r^{2} / 2\right)$ passing through the middle-tropospheric location of maximal $\bar{v}$. (c) Relative humidity (shading) and potential temperature anomaly (red and blue contours), as explained in the caption of Fig. 1b. The dotted black curve corresponds to a relative humidity of $89.4 \%$.

involves redistributing the circulation of each fine grid cell to the larger cells associated with the four nearest coarse grid points by the method of area-weighting, and then dividing the accumulated circulation in each coarse grid cell by $l_{c}^{2}$. Such a procedure effectively smooths out small-scale features in $\zeta$ while conserving the total circulation of the system. The streamfunction $\psi$ and nondivergent velocity field $\mathbf{u}_{\psi}$ associated with the smoothed version of $\zeta$ are computed on the coarse grid and interpolated onto the fine grid. The vortex center $\mathbf{x}_{c}$ is tentatively defined to be the fine grid point where centering a polar coordinate system will maximize the peak value of $\overline{\boldsymbol{v}}_{\psi}(r) \equiv \overline{\hat{\boldsymbol{\varphi}} \cdot \mathbf{u}_{\psi}}$. A minor adjustment to $\mathbf{x}_{c}$ is made to a neighboring half grid point if such a change increases the peak value of $\overline{\boldsymbol{v}}_{\psi}$. The search for $\mathbf{x}_{c}$ is notably limited to regions within the deepest troughs of $\psi$.

Smoothing out small features in $\zeta$ prior to searching for $\mathbf{x}_{c}$ is designed to reduce the probability of associating $\mathbf{x}_{c}$ with the center of an intense but transient meso- $\gamma$-scale vortex. Once a relatively strong tropical storm develops, the probability of such dubious centering without smoothing of $\zeta$ is significantly reduced in the simulations considered for this study. At such a point of development, we therefore eliminate the use of a coarse grid and the smoothing step in the center finding algorithm.

The center of the surface vortex $\mathbf{x}_{c s}$ is generally computed with $h_{-}=0.025 \mathrm{~km}$ and $h_{+}=1.01 \mathrm{~km}$. The center of the middle-tropospheric vortex $\mathbf{x}_{c m}$ is generally computed with $h_{-}=7.34 \mathrm{~km}$ and $h_{+}=8.13 \mathrm{~km}$. The moving reference frame having coordinates centered at $\mathbf{x}_{c s}$ will be called the surface-vortex-centered (SVC) reference frame. After misalignment, the velocity of the SVC reference frame relative to the ES reference frame is typically of order $1 \mathrm{~m} \mathrm{~s}^{-1}$.

Bear in mind that the description of tilt dynamics can vary to some extent with the definition of $\mathbf{x}_{c}$ (e.g., Ryglicki and Hart 2015). The center finding technique

TABLE 1. List of experiments (excluding the control run) and the parameters used to generate the misalignments. See text for discussion.

\begin{tabular}{|c|c|c|c|c|c|c|}
\hline Experiment & $U_{s}\left(\mathrm{~m} \mathrm{~s}^{-1}\right)$ & $\tau_{s}(\mathrm{~h})$ & $2 U_{s} \tau_{s}(\mathrm{~km})$ & $\delta \tau_{s}(\mathrm{~h})$ & $\tau_{d}(\mathrm{~h})$ & $z_{l}(\mathrm{~km})$ \\
\hline IS-X100Z5 & 2.78 & 5 & 100 & 1 & - & 5.25 \\
\hline IS-X200Z5 & 5.56 & 5 & 200 & 1 & - & 5.25 \\
\hline IS-X400Z5 & 11.11 & 5 & 400 & 1 & - & 5.25 \\
\hline ISPD-X100Z5, ISPD-X100Z3 & 2.78 & 5 & 100 & 1 & 1.5 & $5.25,3.5$ \\
\hline ISPD-X200Z5, ISPD-X200Z3 & 5.56 & 5 & 200 & 1 & 1.5 & $5.25,3.5$ \\
\hline ISPD-X250Z5 & 6.94 & 5 & 250 & 1 & 1.5 & 5.25 \\
\hline ISPD-X300Z5, ISPD-X300Z3 & 8.33 & 5 & 300 & 1 & 1.5 & $5.25,3.5$ \\
\hline ISPD-X350Z5 & 9.72 & 5 & 350 & 1 & 1.5 & 5.25 \\
\hline ISPD-X400Z5, ISPD-X400Z3, ISPD-X400Z1 & 11.11 & 5 & 400 & 1 & 1.5 & $5.25,3.5,1.75$ \\
\hline DSPD-X100Z5 & 2.78 & 5 & 100 & 1 & 0.75 & 5.25 \\
\hline DSPD-X200Z5, DSPD-X200Z3 & 2.78 & 10 & 200 & 2 & 1.5 & $5.25,3.5$ \\
\hline DSPD-X300Z5, DSPD-X300Z3 & 2.78 & 15 & 300 & 3 & 1.5 & $5.25,3.5$ \\
\hline DSPD-X400Z5, DSPD-X400Z3 & 2.78 & 20 & 400 & 4 & 1.5 & $5.25,3.5$ \\
\hline
\end{tabular}


described above is presently favored by the authors and (in essence) within the domain of conventional practice. For those interested, appendix B briefly addresses the consequences of using an alternative technique; the contents of this appendix are best read after section $4 \mathrm{a}$.

\section{Impact of misalignment on hurricane formation}

\section{a. Hindered intensification of the maximum surface wind speed}

The central issue considered in this section of the article is the effect of misalignment on the time required for the surface vortex to intensify. The misalignment is quantified by the following tilt vector:

$$
\Delta \mathbf{x}_{c} \equiv \mathbf{x}_{c m}-\mathbf{x}_{c s} .
$$

By definition, $\Delta \mathbf{x}_{c}$ gives the magnitude and direction of the horizontal displacement of the rotational center of the middle-tropospheric vortex from its counterpart at the surface. Our primary measure of tropical cyclone intensity is the maximum value of the azimuthally averaged tangential velocity at the lowest grid level above the ocean, denoted by $v_{m}$. Needless to say, the pertinent value of $v_{m}$ is that measured in the SVC coordinate system.

Let $t_{\text {ITC }}$ denote the time during the evolution of an incipient tropical cyclone (ITC) when $v_{m}$ first reaches a modest pre-tropical storm value of $12.5 \mathrm{~m} \mathrm{~s}^{-1}$. Furthermore, let $t_{\mathrm{CAT} 1}$ denote the time when $v_{m}$ first reaches a value of $32.5 \mathrm{~m} \mathrm{~s}^{-1}$, which approximately corresponds to the threshold wind speed of a category-1 (CAT1) hurricane. Henceforth, the time interval between the aforementioned events will be called the hurricane formation period (HFP). The duration of the HFP is given by $\tau_{\mathrm{hf}}=t_{\mathrm{CAT} 1}-t_{\mathrm{ITC}}$. The time-averaged magnitude of the tilt vector over the HFP will be denoted by "tilt $\mathrm{hf}_{\mathrm{hf}}$ ", It is worth remarking that the value of tilt ${ }_{\mathrm{hf}}$ is closely linked to the maximum tilt magnitude applied within the first $6 \mathrm{~h}$ of simulation time $\left(\right.$ tilt $\left._{0}\right)$. A linear regression yields tilt $\mathrm{hf}_{\mathrm{h}}=-15.066+0.364$ tilt $_{0}(\mathrm{~km})$ and the Pearson correlation coefficient $(\mathrm{PC})$ is $0.88 .^{2}$

Figure 3 shows that $\tau_{\mathrm{hf}}$ reliably grows with tilt $\mathrm{hf}_{\mathrm{h}}$. The linear correlation is quantitatively robust in that $\mathrm{PC}=0.92$. The remainder of section 3 elaborates upon this central result. Sections $3 \mathrm{~b}$ and $3 \mathrm{c}$ examine modifications of the tropical cyclones that coincide with increasing

\footnotetext{
${ }^{2}$ The regression applies to a dataset in which $37 \leq$ tilt $_{0} \leq 367 \mathrm{~km}$ and $10 \leq$ tilt $_{\mathrm{hf}} \leq 122 \mathrm{~km}$. Note also that the truncations of numerical results for the slope and intercept required for presentation (here and elsewhere) are not based on their statistical precisions.
}

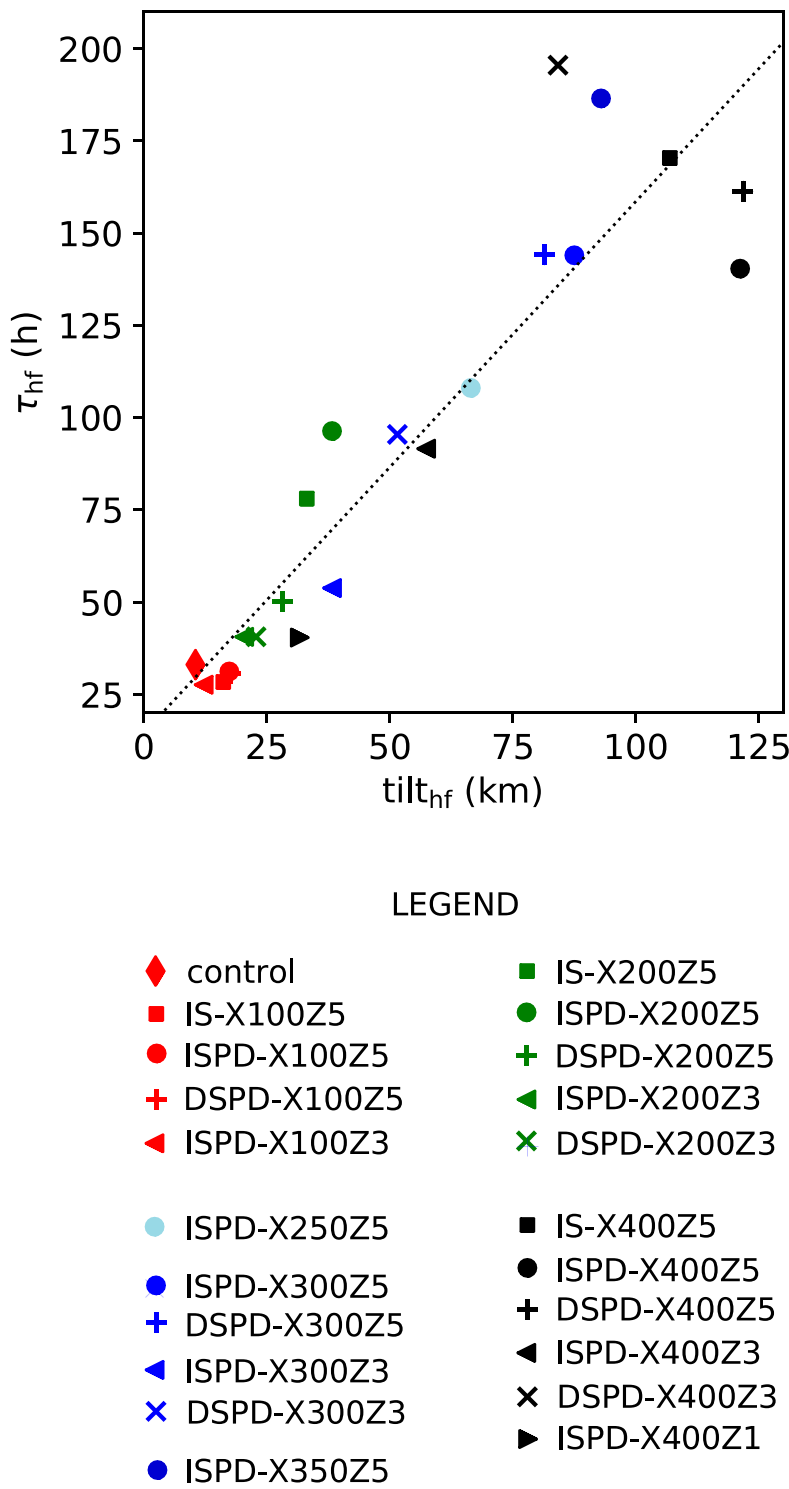

FIG. 3. Relationship between $\tau_{\mathrm{hf}}$ and tilt $\mathrm{hf}_{\mathrm{hf}}$. The dotted linear regression line is given by $\tau_{\mathrm{hf}}=14.569+1.441$ tilt $_{\mathrm{hf}}$ in units corresponding to the plotted data.

tilt magnitudes and slower development. Section $3 \mathrm{~d}$ examines how the changes in vortex structure and moist convection associated with enhanced misalignment affect the angular momentum budget. Section $3 \mathrm{e}$ investigates how increasing the tilt magnitude affects the growth of alternative kinetic-energy-based measurements of vortex intensity.

\section{b. Reorganization of convection and enlargement of the surface vortex}

The slowdown of hurricane formation in a misaligned system coincides with the reorganization of convection and enlargement of the surface vortex. Figure $4 \mathrm{a}$ 

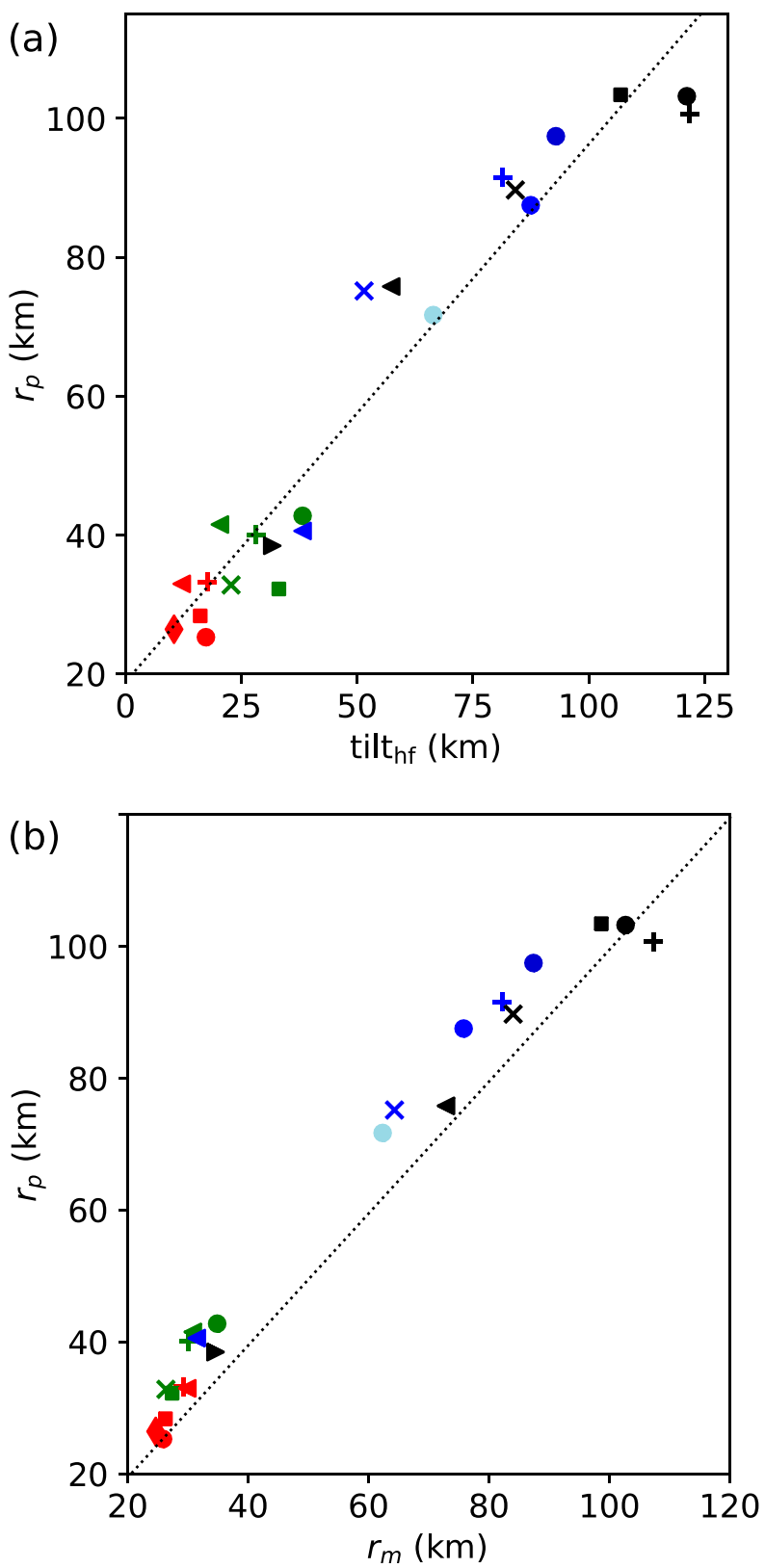

FIG. 4. (a) Precipitation radius $r_{p}$ averaged over the HFP vs tilt $\mathrm{hf}_{\text {. }}$ The dotted linear regression line is given by $r_{p}=18.825+0.775$ tilt $_{\mathrm{hf}}$ $(\mathrm{km})$ and the correlation coefficient is 0.96. (b) Relationship between $r_{p}$ and the radius of maximum surface- $\bar{v}$, denoted $r_{m}$, averaged over the HFP. The dotted reference line corresponds to $r_{p}=r_{m}$. See Fig. 3 for the symbol legend.

demonstrates that greater values of tilt $\mathrm{h}_{\mathrm{hf}}$ correspond to greater values of the time-averaged precipitation radius $r_{p}$. By definition, $r_{p}$ is the radius in the SVC coordinate system at which the azimuthally averaged 2 -h precipitation (surface rainfall) distribution is maximized. Figure $4 \mathrm{~b}$ shows that the time average of the radius $r_{m}$ at which $v_{m}$ occurs grows commensurately with $r_{p}$.
In addition to moving outward, the precipitation grows increasingly asymmetric with enhanced tilt. Figure 5 depicts the tilt $\mathrm{hf}_{\mathrm{hf}}$ dependence of the 2-h precipitation asymmetry during the HFP. The total (area integrated) 2 -h precipitation in a $400-\mathrm{km}$ circular disc centered at $\mathbf{x}_{c s}$ is split into individual contributions from 4 quarter circles. The quarter circles are centered in azimuth at $\varphi=0^{\circ}, 90^{\circ}, 180^{\circ}$, and $270^{\circ}\left(-90^{\circ}\right)$, with $\varphi=0$ corresponding to the downtilt direction (i.e., the direction of $\left.\Delta \mathbf{x}_{c}\right)$. Each absolute contribution to the 2-h precipitation is then divided by the total to form a fractional contribution. The plotted precipitation probability is the time average of the fractional contribution over the HFP. As tilt ${ }_{\mathrm{hf}}$ increases from 10 to $60 \mathrm{~km}$, the probability of precipitation in the downtilt quadrant dramatically grows from slightly above $25 \%$ to approximately $60 \%$. The probability of precipitation in the uptilt quadrant (centered at $180^{\circ}$ ) decays to a value substantially less than $10 \%$. The precipitation probabilities in the quadrants centered at $-90^{\circ}$ and $90^{\circ}$ also diminish, but the former decays less than the latter.

For illustrative purposes, Figs. 6 and 7 depict the asymmetric structure of the vortex in simulation DSPDX400Z5 at a time during the HFP when $\left|\Delta \mathbf{x}_{c}\right|=240 \mathrm{~km}$. DSPD-X400Z5 is among a handful of simulations most worthy (in our view) of detailed examination, because the misalignment coincides with severely hindered development of the tropical cyclone. The structure of the vortex in DSPD-X400Z5 is similar to that found in earlier studies of real-world and simulated tropical cyclones that are tilted by moderate environmental wind shear prior to achieving hurricane status (e.g., Rappin and Nolan 2012; Nguyen et al. 2017). Figure 6 shows the misalignment of quasi-circular lower-tropospheric (lw) and middle-tropospheric (md) streamlines in the slowly moving SVC reference frame, superimposed on a complementary plot of the magnitude of the local shear velocity, defined by $\mathbf{u}_{\mathrm{md}}-\mathbf{u}_{\mathrm{lw}}{ }^{3}$ The lower- and middletropospheric flows specifically correspond to elevations of 1.2 and $7.7 \mathrm{~km}$ above sea level. The green ray represents the $\varphi=0$ axis and therefore points exactly downtilt.

Figure $7 \mathrm{a}$ shows the 2-h precipitation field rotated such that downtilt is now directly to the right. Consistent with Fig. 5, much of the precipitation is seen in the downtilt quadrant, whereas the uptilt quadrant is

\footnotetext{
${ }^{3}$ A qualitatively similar shear pattern but with a smaller maximum magnitude of $22 \mathrm{~m} \mathrm{~s}^{-1}\left(19 \mathrm{~m} \mathrm{~s}^{-1}\right)$ is seen when $\mathbf{u}_{\mathrm{md}}$ and $\mathbf{u}_{\mathrm{lw}}$ are first smoothed using a Gaussian kernel with a standard deviation of $30 \mathrm{~km}(60 \mathrm{~km})$ in both $x$ and $y$. A similar pattern is also seen at $t=0$, prior to any convection in experiment DSPD-X400Z5, but with a peak shear magnitude of $14 \mathrm{~m} \mathrm{~s}^{-1}$ more directly between the lowerand middle-tropospheric centers of rotation.
} 


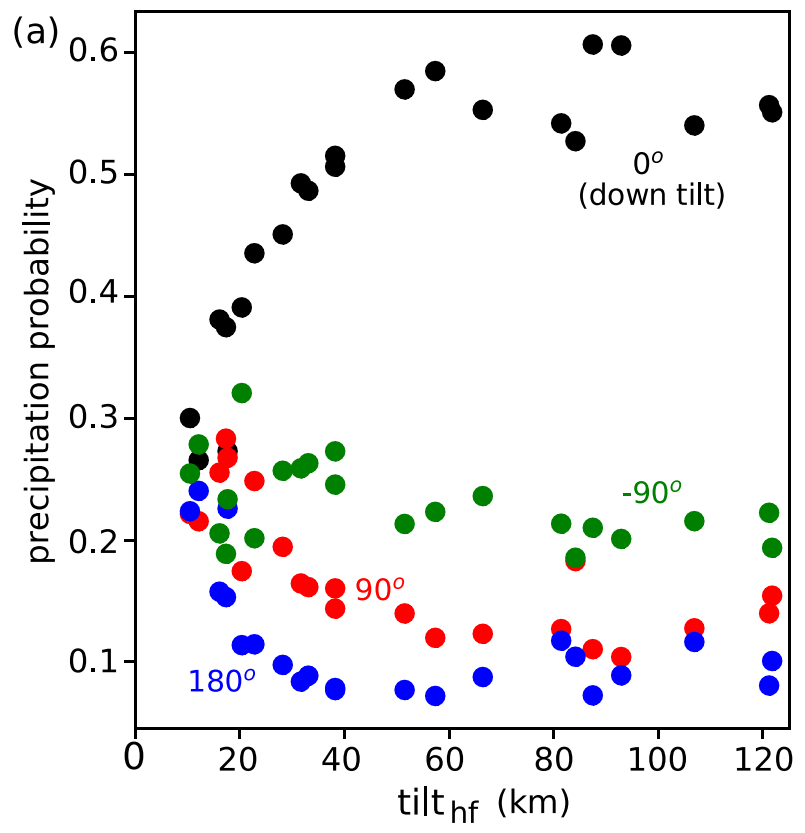

(b)

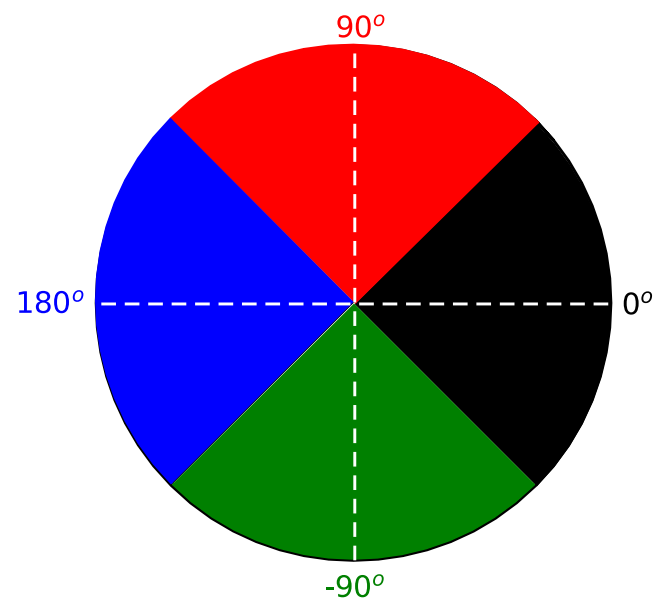

FIG. 5. (a) Variation of the precipitation probability distribution

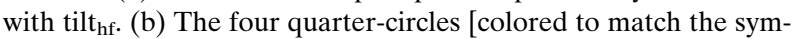
bols in (a)] over which the precipitation probability is distributed; the angles are defined such that the time-dependent tilt vector $\Delta \mathbf{x}_{c}$ always points toward $0^{\circ}$.

relatively quiet. Figure $7 \mathrm{~b}$ shows the surface streamlines superimposed on the boundary layer equivalent potential temperature $\theta_{\mathrm{eb}}$, defined here as the vertical average over the lowest $1 \mathrm{~km}$ of the troposphere. The distribution of $\theta_{\mathrm{eb}}$ has relatively low values at and downwind of where low-entropy downdrafts are expected in association with strong convective activity. Note that the deficit of $\theta_{\mathrm{eb}}$ in the vicinity of the active precipitation region coincides with a pronounced cold pool having surface values of ordinary potential temperature $\theta$ down to $3.3 \mathrm{~K}$ below the domain average (not shown). Figure $7 \mathrm{c}$ shows the horizontal distribution of

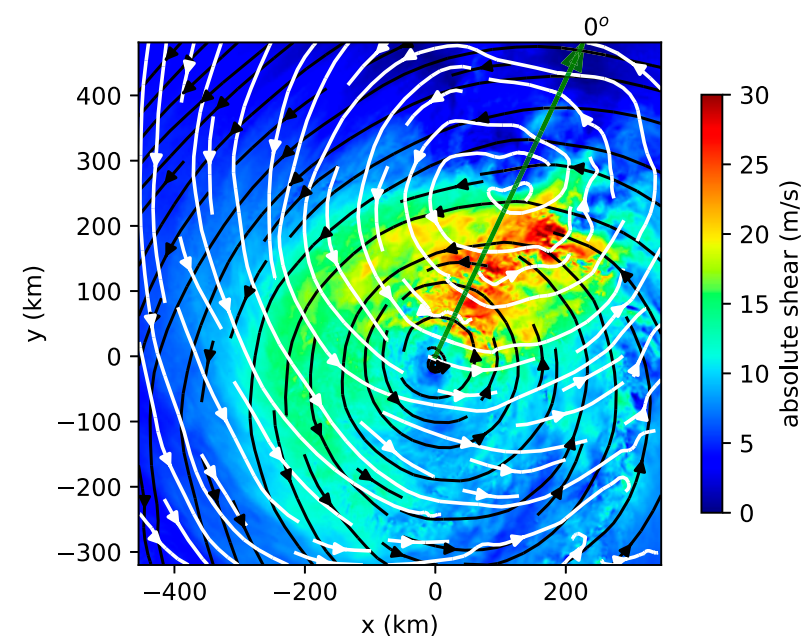

FIG. 6. Depiction of the horizontal flow in the SVC reference frame $48 \mathrm{~h}$ after the initial misalignment in simulation DSPDX400Z5. The black and white streamlines respectively correspond to $z=1.2$ and $7.7 \mathrm{~km}$. Colors show the magnitude of the local shear velocity measured between the two levels. The green ray points in the direction of the tilt vector.

relative humidity averaged between $z=2.3$ and $7.7 \mathrm{~km}$, taking values with respect to ice/liquid at altitudes above/below the freezing level. Humidification that could facilitate vigorous deep convection has failed to develop uptilt. Figure $7 \mathrm{~d}$ depicts a lowermiddle-tropospheric flow pattern suggesting that an influx of relatively dry air from the outer part of the vortex and a weak meso- $\alpha$-scale downdraft (in conjunction with subsidence warming) contribute to maintaining low relative humidity uptilt.

Although more than one factor may contribute to the predominant downdraft between $\varphi=0$ and $180^{\circ}$, its magnitude notably agrees with an estimate that assumes middle-tropospheric downgliding of unsaturated air along a nearly frozen virtual potential temperature isosurface. Figure 7e depicts one such isosurface in the CM1 simulation, which compares favorably to that of a system with equivalent $\zeta$ that has adjusted to a state of nonlinear balance (Fig. 7f, see appendix C). The downgliding velocity is estimated as $w_{\mathrm{dg}} \sim \delta z U / L=-0.01 \mathrm{~m} \mathrm{~s}^{-1}$, in which $\delta z \sim-5 \times 10^{2} \mathrm{~m}$ is the vertical displacement along a streamline path that traverses the interval $0^{\circ} \leq \varphi \leq 180^{\circ}, U \sim 10 \mathrm{~m} \mathrm{~s}^{-1}$ is the characteristic horizontal wind speed, and $L \sim 5 \times 10^{5} \mathrm{~m}$ is the horizontal pathlength.

\section{c. Variation of moist-thermodynamic parameters}

The convective and structural modifications associated with misalignment coincide with changes to several bulk moist-thermodynamic parameters in the surface-centered core of the developing system. 

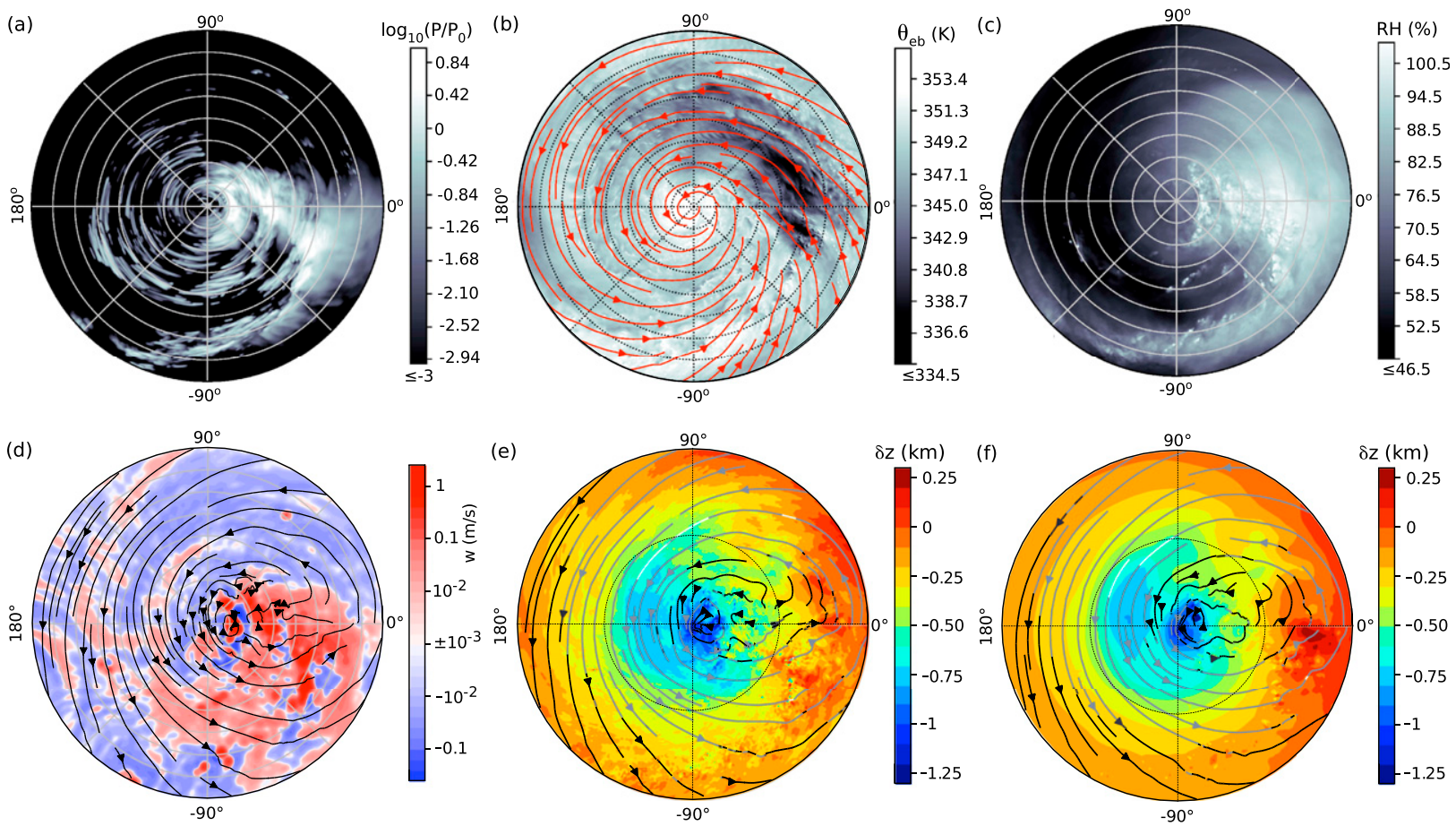

FIG. 7. Selected fields associated with convection $48 \mathrm{~h}$ after the initial misalignment in simulation DSPD-X400Z5. All fields are rotated such that the tilt vector points directly to the right. (a) Logarithm of the 2 -h accumulated precipitation $P$ normalized to $P_{0}=1 \mathrm{~cm}$. The accumulation is measured over the interval $47 \leq t \leq 49 \mathrm{~h}$. (b) Vertical average of equivalent potential temperature in the 1-km surface boundary layer, denoted $\theta_{\mathrm{eb}}$. Surface streamlines are shown in red. (c) Vertical average of the relative humidity between $z=2.3$ and $7.7 \mathrm{~km}$. (d) Vertical velocity field (red and blue) and streamlines of the horizontal flow (black) in the lower-middle troposphere. The vertical velocity field $w$ is averaged between $z=2.3$ and $7.7 \mathrm{~km}$, and is smoothed in the horizontal plane using a Gaussian kernel with a standard deviation of $6.2 \mathrm{~km}$ in both dimensions. The colormap is logarithmic. The streamlines correspond to $z=5.6 \mathrm{~km}$ and thus belong to a transition layer where the center of rotation is in between $\mathbf{x}_{c s}$ and $\mathbf{x}_{c m}$. (e),(f) Height perturbation about $z=5.52 \mathrm{~km}$ (color) and horizontal streamlines on the $\theta_{v}=325.4-\mathrm{K}$ isosurface in (e) the CM1 simulation and (f) a system with equivalent $\zeta$ in a state of nonlinear balance. The streamlines in (e) and (f) are shaded such that black indicates $|\mathbf{u}|<7.5 \mathrm{~m} \mathrm{~s}^{-1}$, gray indicates $7.5 \leq|\mathbf{u}| \leq 15 \mathrm{~m} \mathrm{~s}$ and white indicates $|\mathbf{u}|>15 \mathrm{~m} \mathrm{~s}^{-1}$. All streamlines in (b) and (d)-(f) correspond to $\mathbf{u}$ in the slowly moving SVC reference frame. Grid circles are spaced $50 \mathrm{~m}$ apart in (a)-(d); the dotted circles in (e) and (f) show where $r=200 \mathrm{~m}$.

Figure $8 \mathrm{a}$ verifies that strong negative correlations $(\mathrm{PC}=-0.92 \pm 0.01)$ exist between tilt ${ }_{\mathrm{hf}}$ and spatiotemporal averages of the relative humidity distribution measured as in Fig. 7c. The time averaging covers the entire HFP. The spatial averaging is over a cylindrical volume defined in the SVC coordinate system by $2.3 \leq z \leq 7.7 \mathrm{~km}$ and $0 \leq r \leq R$, in which $R$ is either 100 or $250 \mathrm{~km}$. Qualitatively similar anticorrelations have been verified for $R=25$ and $400 \mathrm{~km}$ (not shown). The association of enhanced tilt with drier air above the surface vortex is notable in view of prior studies suggesting that such low humidity alone can hinder the onset of rapid intensification (e.g., section 3 of Schecter 2016).

One might reasonably ask whether slower development in a strongly misaligned system also coincides with a reduction in the rate at which the vortex extracts the sum of latent and sensible heat from the sea surface. Figure $8 \mathrm{~b}$ addresses the preceding question by showing the relationship between the tilt magnitude and the parameterized surface enthalpy flux. The enthalpy flux $F_{k}$ is defined as in Eq. (3) of Zhang et al. (2008). As for relative humidity, the plotted value of $F_{k}$ is a spatiotemporal mean taken within a variable radius $R$ of the surface vortex center during the HFP. A strong anticorrelation $(\mathrm{PC}=-0.93)$ exists between the mean value of $F_{k}$ and tilt $\mathrm{hf}_{\mathrm{hf}}$ when $R=100 \mathrm{~km}$; a qualitatively similar result is found for $R=25 \mathrm{~km}$ (not shown). The anticorrelation becomes far less convincing for broader averaging discs, as shown for the case in which $R=250 \mathrm{~km}$, where $\mathrm{PC}=-0.44$

Note that slower development with enhanced tilt is not associated with a reduction of convective available potential energy (CAPE) in the central region of the surface vortex. Figure 8c shows the spatiotemporal mean value of the 500-m mixed layer CAPE, calculated under the assumption of undiluted pseudoadiabatic ascent 

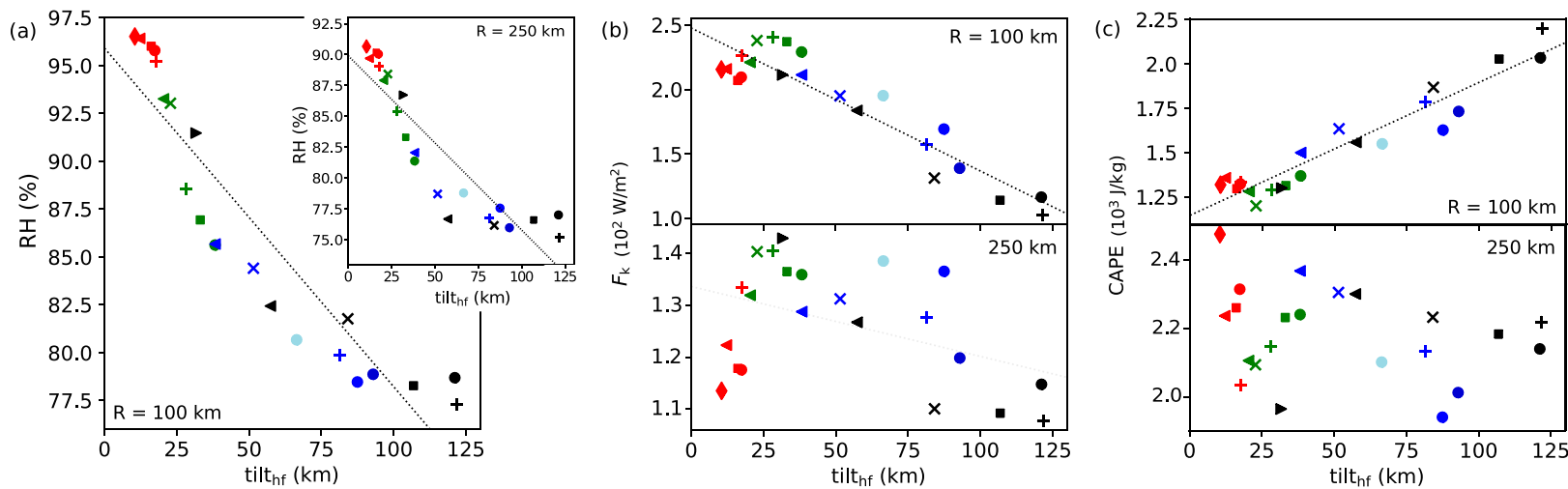

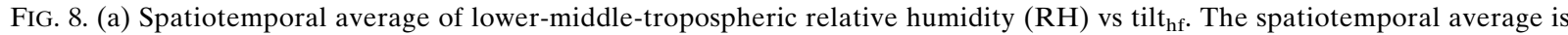
within a radius $R$ of $100 \mathrm{~km}$ (main plot) or $250 \mathrm{~km}$ (inset) from $\mathbf{x}_{c s}$ during the HFP. The dotted linear regression line is given by RH $=$ $95.899-0.177$ tilt $_{\mathrm{hf}}$ (main plot) or RH $=89.896-0.141$ tilt $\mathrm{th}_{\mathrm{hf}}$ (inset). (b) Similar spatiotemporal average of the surface enthalpy flux $F_{k}$ vs tilt $_{\text {hf }}$ for (top) $R=100 \mathrm{~km}$ and (bottom) $R=250 \mathrm{~km}$. The dotted linear regression line in the top plot is given by $F_{k}=2.476-0.011$ tilt (c) Similar spatiotemporal average of CAPE vs tilt hf for (top) $R=100 \mathrm{~km}$ and (bottom) $R=250 \mathrm{~km}$. The dotted linear regression line in the top plot is given by CAPE $=1.146+0.008$ tilt $_{\mathrm{hf}}$. Note that the units of the variables in each regression equation equal those in the corresponding plot. See Fig. 3 for the symbol legend.

with liquid-only condensate. The averaging is identical to that of $F_{k}$. For $R=100 \mathrm{~km}$, the mean CAPE reliably grows $(\mathrm{PC}=0.95)$ with increasing values of tilt $\mathrm{h}_{\mathrm{hf}}$; the same is true when $R$ is reduced to $25 \mathrm{~km}$ (not shown). When $R$ is extended to $250 \mathrm{~km}$, no meaningful correlation between the mean CAPE and tilt $\mathrm{h}_{\mathrm{hf}}$ can be established $(\mathrm{PC}=-0.26)$.

\section{d. Modification of the angular momentum budget}

It is now appropriate to delve deeper into how the geometrical restructuring of the vortex and the coupled reorganization of convection affect the mechanism of surface spinup in the vicinity of maximal winds. A comprehensive investigation would extend beyond the scope of this article, but a limited analysis seems fitting. The following compares the $\bar{v}$ budget of DSPD-X400Z5 (in the SVC reference frame) to that of the control simulation during 6 -h periods of early development when the two systems have comparable mean values of $v_{m}$ but distinct temporal trends. Whereas DSPD-X400Z5 shows a slightly negative trend, the control simulation shows substantial intensification (Fig. 9).

Figure 10 depicts the time-averaged state of each system during the aforementioned 6-h analysis periods. In the azimuthal mean, the misaligned vortex with highly asymmetric convection (DSPD-X400Z5) has a shallower cyclonic circulation, a larger value of $r_{m}$, and two distinct updrafts sprouting from the lower troposphere. The virtually symmetric convection in the control simulation is characterized by a single strong updraft peaked near the relatively small radius of maximum surface wind speed.
The tendency of $\bar{v}$ is analyzed as explained in appendix D. ${ }^{4}$ The analysis involves decomposing the mean secondary circulation as follows:

$$
\left(\begin{array}{c}
\bar{u} \\
\bar{w}
\end{array}\right) \stackrel{\text { theory }}{=}\left(\begin{array}{c}
\bar{u}_{\mathrm{mp}}+\bar{u}_{e}+\bar{u}_{\mathcal{T}} \\
\bar{w}_{\mathrm{mp}}+\bar{w}_{e}+\bar{w}_{\mathcal{T}}
\end{array}\right),
$$

in which the terms on the right-hand side are associated with different forcings in the Sawyer-Eliassen (SE) equation (e.g., Smith et al. 2005; Schubert and Hack 1982; Shapiro and Willoughby 1982). The mp-circulation derives from the heating (positive and negative) associated with cloud microphysics. The $e$-circulation derives from resolved eddy forcing. The $\mathcal{T}$-circulation derives from unresolved (parameterized) turbulence and several other factors mentioned in appendix $\mathrm{D}$. The change in the velocity field over the analysis period of length $\delta t$ is given by

$$
\delta \bar{v}^{\text {theory }} \delta t \tilde{\mathcal{A}}_{\mathrm{mp}}+\delta t \tilde{\mathcal{A}}_{e}+\delta t \tilde{\mathcal{A}}_{\mathcal{T}}+\delta t\left\langle\overline{\mathcal{E}}_{v}\right\rangle_{t}+\delta t\left\langle\overline{\mathcal{T}}_{v}\right\rangle_{t},
$$

in which $\tilde{\mathcal{A}}_{\alpha}$ is an approximation of the time-averaged tendency associated with angular momentum transport by the $\alpha$-circulation, $\left\langle\overline{\mathcal{E}}_{v}\right\rangle_{t}$ is the time-averaged eddy forcing of $\bar{v}$, and $\left\langle\overline{\mathcal{T}}_{v}\right\rangle_{t}$ is the time-averaged forcing of $\bar{v}$ associated with unresolved turbulence. Figures 11a and 11c demonstrate that the right-hand side of Eq. (5) agrees reasonably well with the temporal mean of the

\footnotetext{
${ }^{4}$ The reader is encouraged to consult appendix D not only for mathematical details, but also for some guidance on interpreting the equations presented herein.
} 


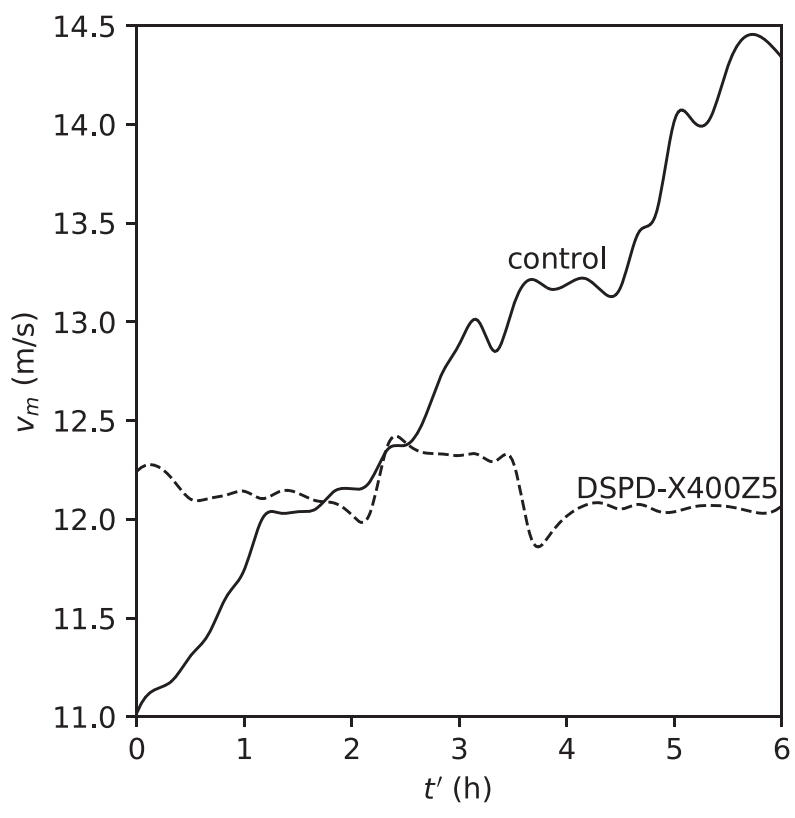

FIG. 9. Time series of $v_{m}$ in simulation DSPD-X400Z5 (dashed) and the control experiment (solid) during the 6-h periods of early development depicted in Figs. 10-12. Time $t^{\prime}$ is measured from the start of each 6-h period.

actual secondary circulation in both CM1 simulations under present consideration. Figures $11 \mathrm{~b}$ and $11 \mathrm{~d}$ furthermore validate Eq. (6).

Figures $12 \mathrm{a}-\mathrm{d}$ show the individual contributions to $\delta \bar{v}$ [Eq. (6)] in simulation DSPD-X400Z5. It is seen that the mp-circulation acts to broadly accelerate the cyclonic winds in the lower troposphere. If acting alone, the mpcirculation would boost the near-surface values of $\bar{v}$ by up to $5 \mathrm{~m} \mathrm{~s}^{-1}$ in the neighborhood of the maximal winds of the time-averaged vortex. Of course, other factors are equally important to the azimuthal velocity budget. Stronger positive and negative tendencies are found near the surface in association with the $\mathcal{T}$-circulation and direct forcing by unresolved turbulent transport; their combination (not shown) is predominantly negative. The tendencies associated with the $e$-circulation and direct eddy forcing near the maximal surface winds are smaller but relevant.

Figures $12 \mathrm{e}-\mathrm{h}$ show the contributions to $\delta \bar{v}$ in the control simulation. Here one finds that the mp-circulation more vigorously accelerates the cyclonic winds of the inner core. If left uncontested, the mp-circulation would boost the near surface values of $\bar{v}$ by $9-10 \mathrm{~m} \mathrm{~s}^{-1}$ slightly outward of the maximal winds of the time-averaged vortex. The positive and negative contributions from $\delta t \tilde{\mathcal{A}}_{\mathcal{T}}$ and $\delta t\left\langle\overline{\mathcal{T}}_{v}\right\rangle_{t}$ near the surface are qualitatively similar to their counterparts in the misaligned system, and are again net-negative (not shown) in the vicinity of maximal $\bar{v}$.
In the same region, $\delta t \tilde{\mathcal{A}}_{e}$ and $\delta t\left\langle\overline{\mathcal{E}}_{v}\right\rangle_{t}$ are appreciable but mutually opposing.

Perhaps the main result from the foregoing analysis is that the restructuring of the vortex and the reorganization of convection in the misaligned system rendered the mp-circulation less effective in accelerating the maximum of $\bar{v}$ near the sea surface. In the case at hand, such reduced efficiency allowed the net negative contribution from other factors in the azimuthal velocity budget (which stayed sufficiently strong) to completely nullify the growth of $v_{m}$.

Note that reasonable accuracy of the SE-based analysis relied partly on the small fractional difference $(0.3$ or less) between the gradient wind and $\bar{v}$ in the vicinity of the surface maximum. The error may worsen considerably at a later stage of development if the degree of gradient imbalance were to intensify in the boundary layer, as often occurs in simulations of tropical cyclones (e.g., Bui et al. 2009; Montgomery and Smith 2014; Schecter 2016). In an alternative experiment where tilt is introduced at such a time, the detrimental effect of the associated convective asymmetry on intensification of $v_{m}$ might be compounded by limiting supergradient flow (cf. Schecter 2013). A separate study would be required to shed light on this issue.

\section{e. Surface kinetic energy growth}

One might wonder whether increasing the tilt magnitude has the same qualitative effect on all measures of vortex intensity. Herein, we address the preceding question by comparing time series of several intensity parameters. Figure 13 (left) shows the temporal growth of $v_{m}$ for all simulations over the time scale for the virtually aligned control vortex to mature into a well-developed hurricane. Each thick curve covers the spread in a group of vortices that are initially perturbed with similar target misalignments $\left(2 U_{s} \tau_{s}\right)$ and equivalent values of $z_{l}$. Consistent with Fig. 3, the time series exhibit considerable variation; enhanced tilt markedly slows the intensification of $v_{m}$.

Alternatively, one might consider the temporal amplification of kinetic energy. Neglecting minor density variations, the kinetic energy contained in the primary component of the surface circulation over the interval $R_{1} \leq r \leq R_{2}$ is directly proportional to

$$
V_{s}^{2} \equiv \frac{2}{R_{2}^{2}-R_{1}^{2}} \int_{R_{1}}^{R_{2}} d r r \bar{v}_{s}^{2}
$$

in which $\bar{v}_{s}$ denotes $\bar{v}$ measured in the SVC coordinate system at the first grid level above the ocean. The center panels of Fig. 13 show $V_{s}^{2}$ parameterized with $R_{1}=0$ and $R_{2}=75 \mathrm{~km}$, so as to represent the kinetic energy of the 

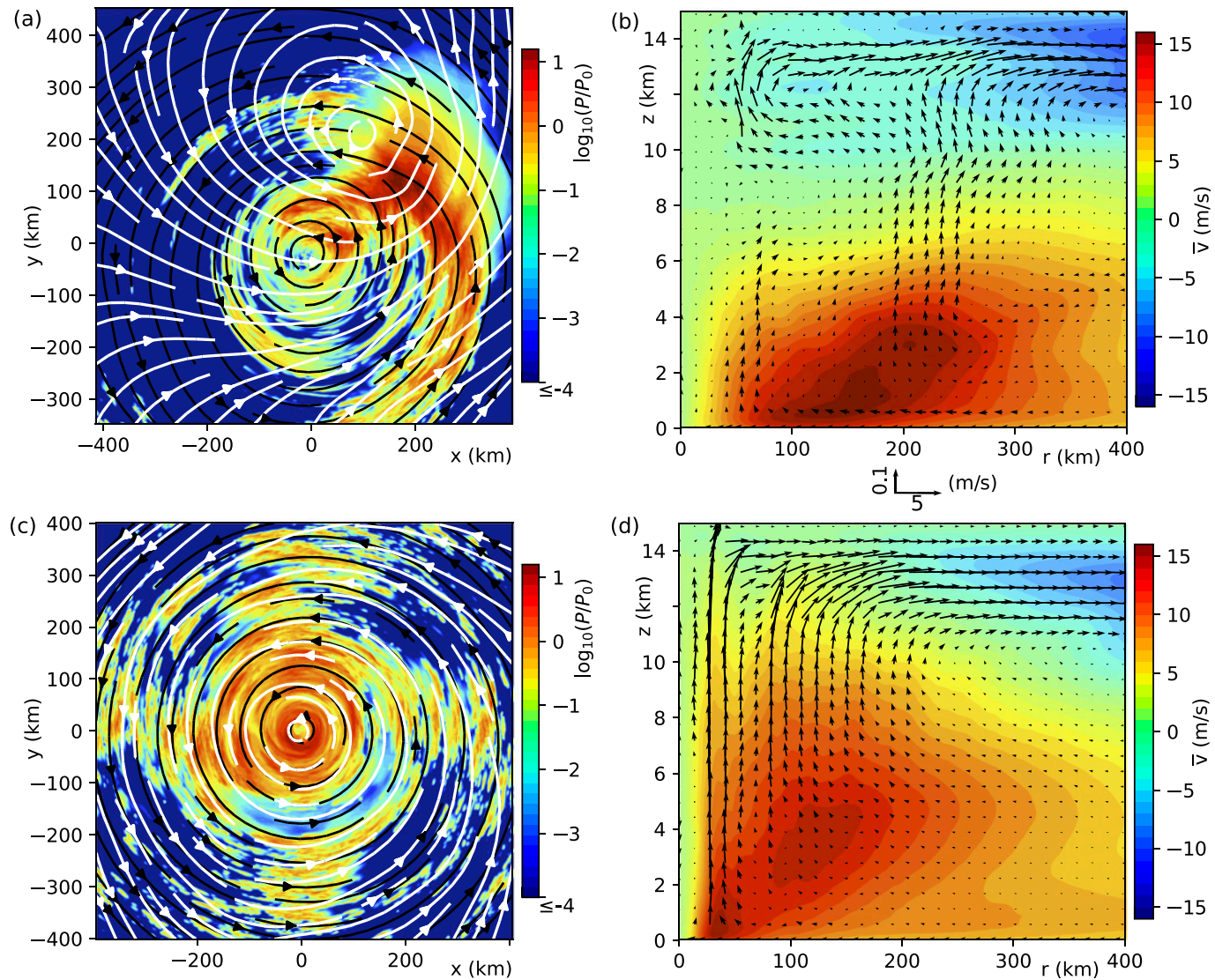

FIG. 10. (a),(b) Structure of the tropical cyclone in simulation DSPD-X400Z5 during a selected 6-h period of early development ( $37 \leq t \leq 43 \mathrm{~h}$ ). (a) Streamlines of the 6-h time-averaged horizontal flow in the SVC reference frame at $z=1.2 \mathrm{~km}$ (black) and $z=7.7 \mathrm{~km}$ (white) superimposed over the logarithm of the 6-h accumulated precipitation $P$ normalized to $P_{0}=1 \mathrm{~cm}$. (b) The 6-h time averages of $\bar{v}$ (color) and the vector velocity field $(\bar{u}, \bar{w})$ associated with the secondary circulation. (c),(d) As in (a) and (b), but for the control experiment over a time interval $(9 \leq t \leq 15 \mathrm{~h})$ with a comparable mean value of $v_{m}$.

inner circulation. The time series show variations similar to those of $v_{m}$. The picture changes dramatically upon considering the kinetic energy of the outer circulation. The right panels of Fig. 13 show $V_{s}^{2}$ parameterized with $R_{1}=75 \mathrm{~km}$ and $R_{2}=750 \mathrm{~km}$. The growth trends hardly differ from one another following an early adjustment period, regardless of the magnitude of the misalignment. Thus, the detrimental effect of misalignment on surface kinetic energy growth is confined to the inner region of the surface vortex over the time period under present consideration.

\section{Tilt dynamics}

Because tropical cyclones with minimal misalignment are generally more efficient in accelerating the cyclonic surface winds, understanding how tilt decays is an important part of understanding intensification. Figure 14 shows that the evolution of tilt is generally nonmonotonic in systems with initially forced tilt magnitudes exceeding approximately $150 \mathrm{~km} .{ }^{5}$ To facilitate discussion, we divide the evolution into three consecutive stages. Stage 1 involves a rapid reduction of the misalignment. Stage 2 entails partial regrowth of the tilt magnitude. Stage 3 is eventually characterized by gradual decay of the tilt magnitude, but may begin with a repetition of the preceding cycle (see, e.g., the dotted curve in Fig. 14b). The remainder of this section will examine the three stages of evolution in detail for simulation DSPD-X400Z5, which is distinguished by having the largest initial tilt. The pathways of tilt decay and transient amplification that operate in DSPD-X400Z5 are considered illustrative of

\footnotetext{
${ }^{5}$ Note that each data group represented by a thick curve includes IS and/or ISPD simulations whose tilts are amplified through artificial forcing over the interval $0 \leq t \leq 6 \mathrm{~h}$, and DSPD simulations whose tilts are imposed and set free at $t=0$.
} 

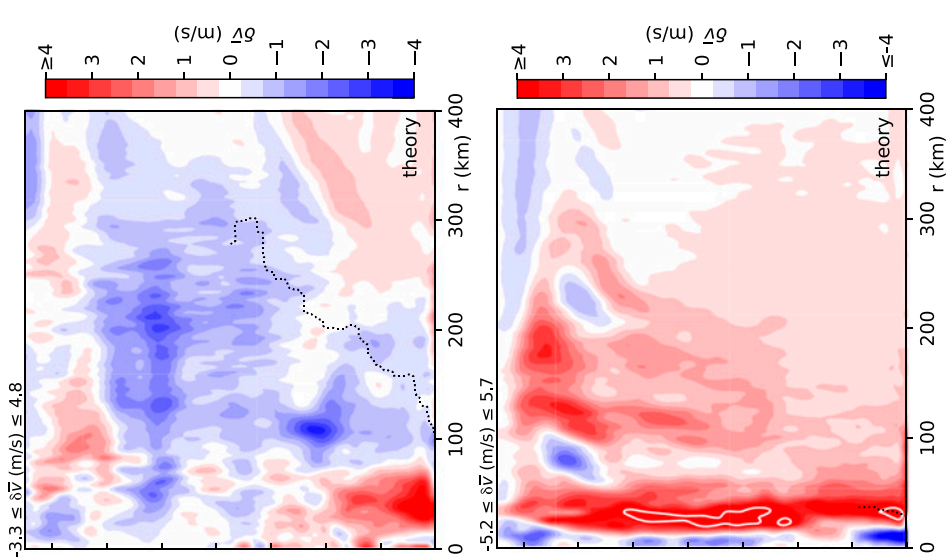

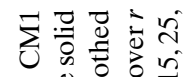

os

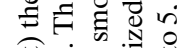

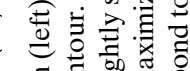
$\exists$ : की

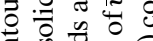
8 过 0 . 获 范 它 落定自

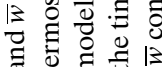
产自至造造
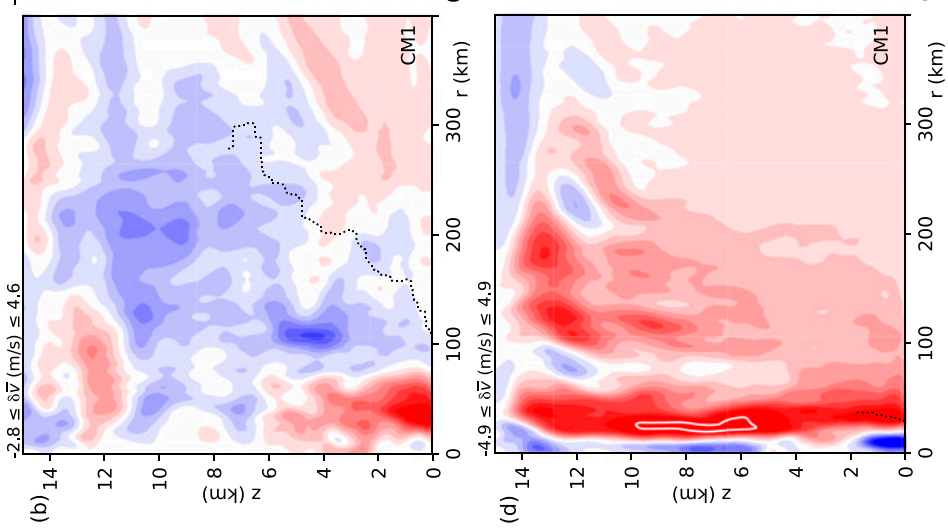
过 ₹ s.

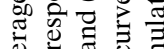

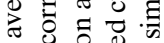

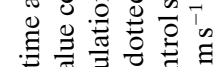

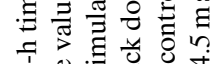

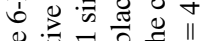

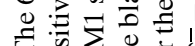

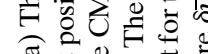
त

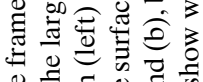
$8 \Xi \cong$ क ज

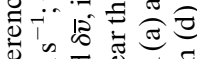
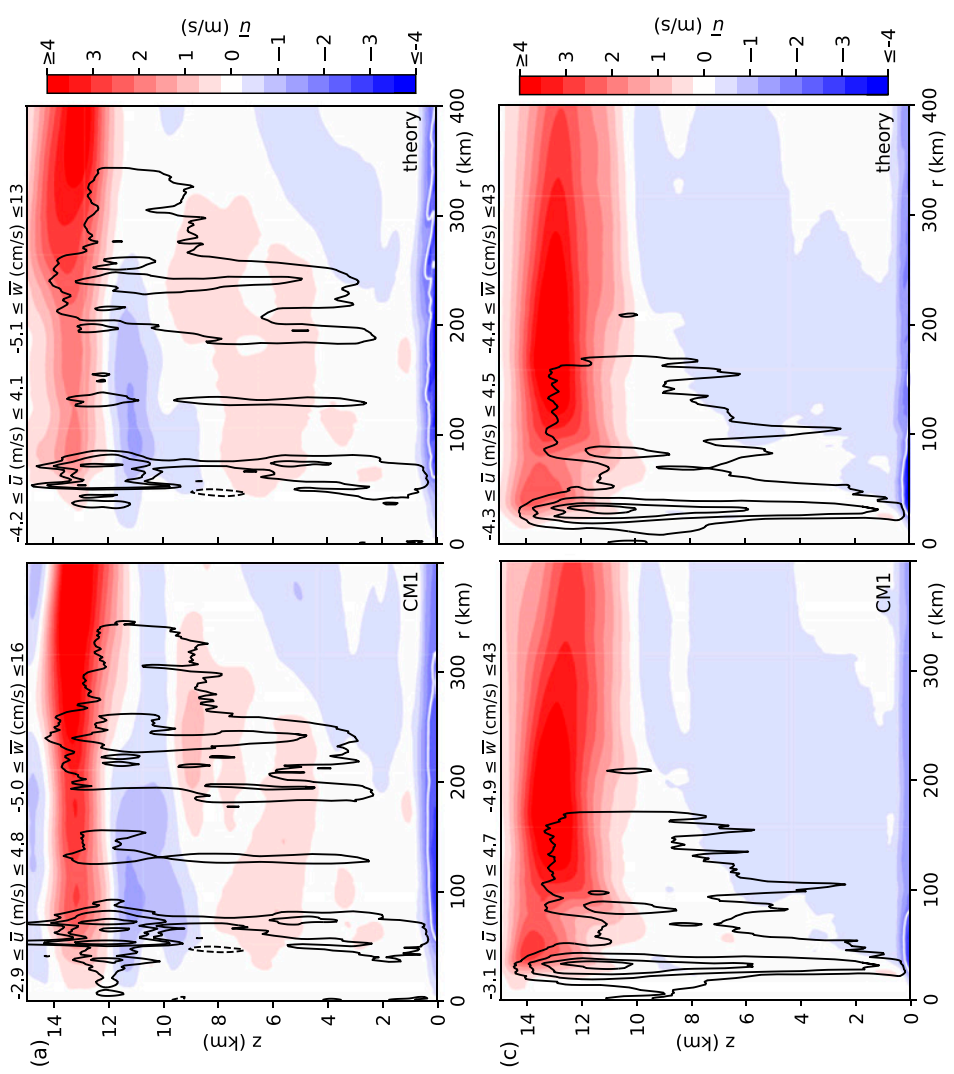

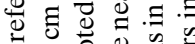

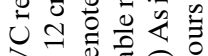
is च च

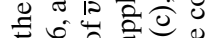
虽 讨 践

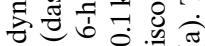

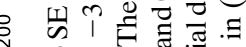

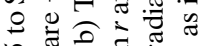

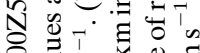

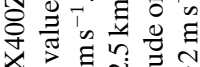

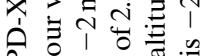
के

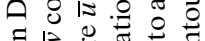

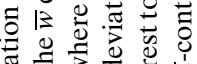

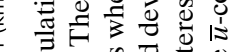
-

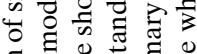

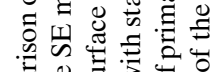
영

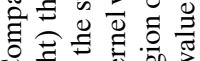
0 क का क ज อ. తิ

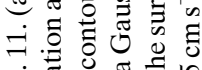

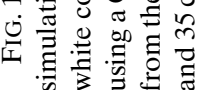



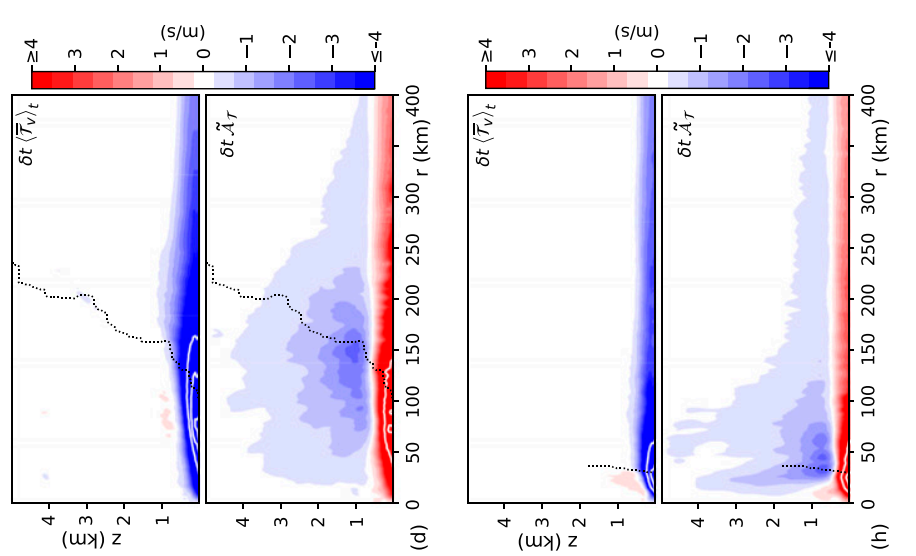

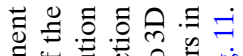

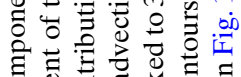

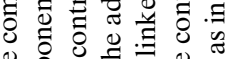

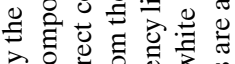
उठ유.

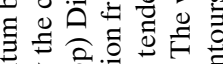
苛产过

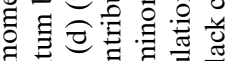

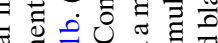

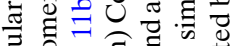

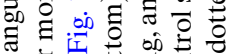

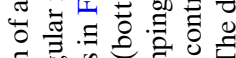
ธิ

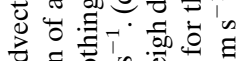
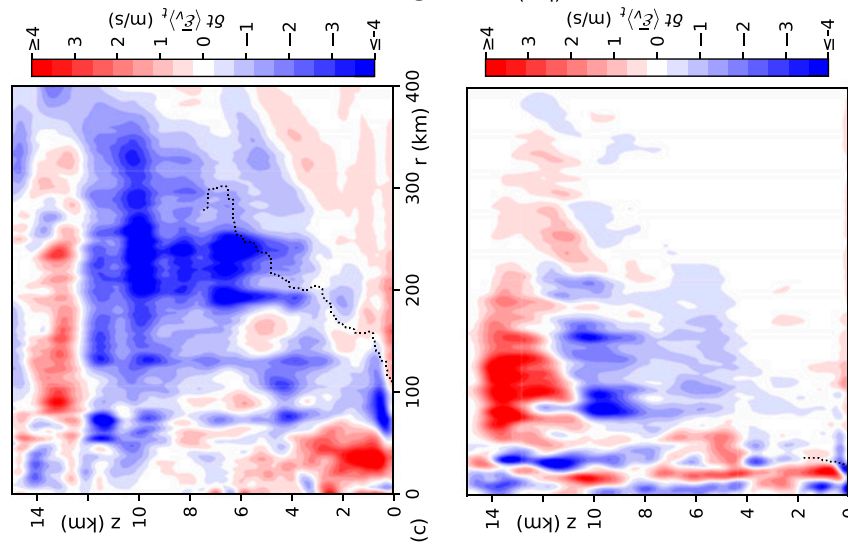

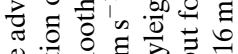

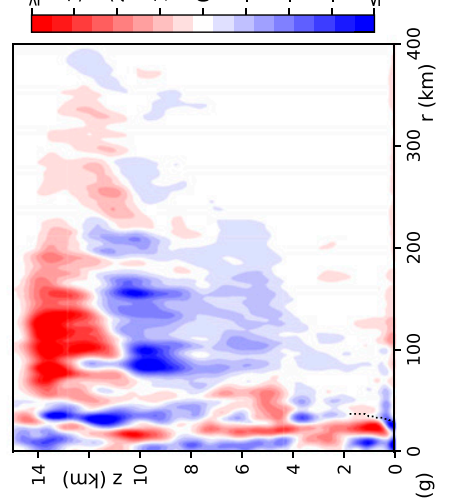
记 造步

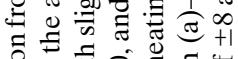

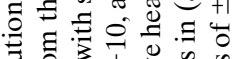

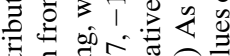

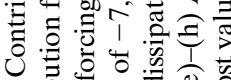

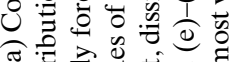

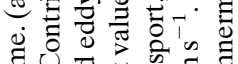

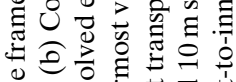

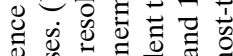

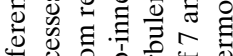

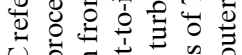
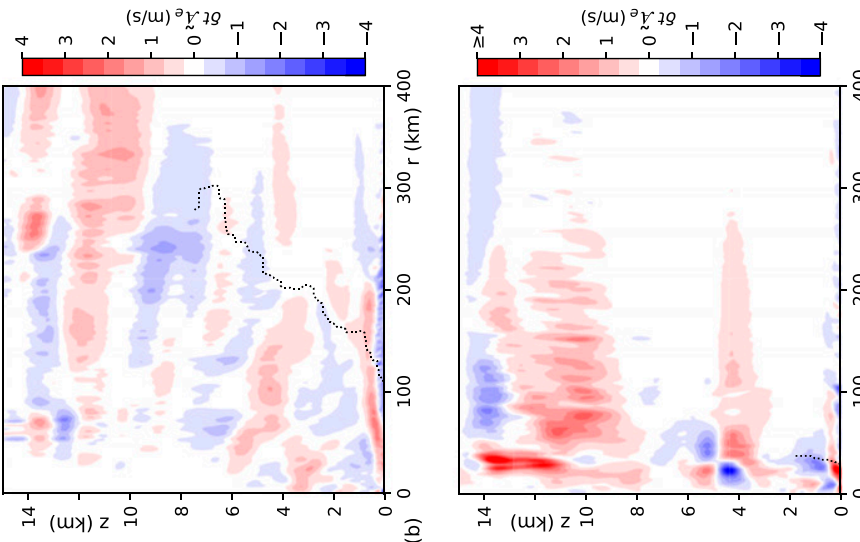

U⿺辶万人

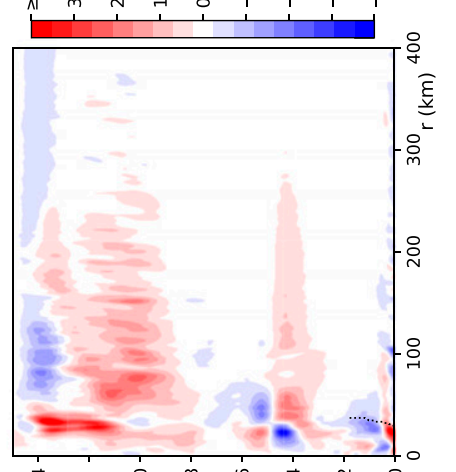

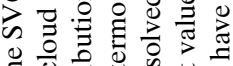

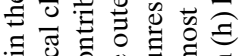
in

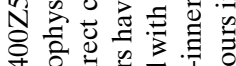

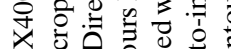

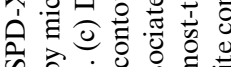

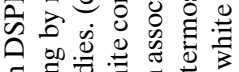

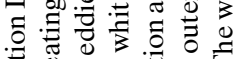
可 寻

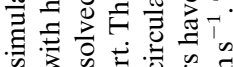

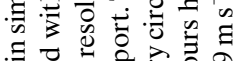

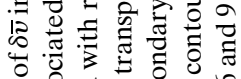
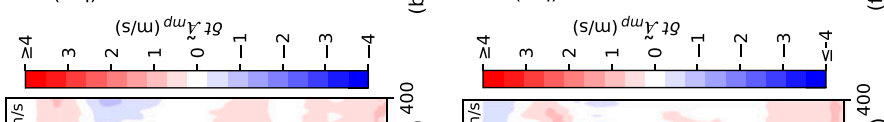

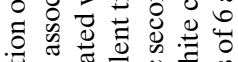
㻤

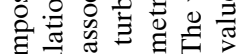

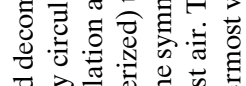
ठ

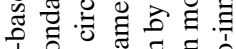

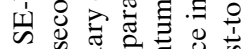
O. 象 త 过

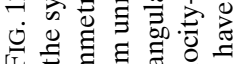

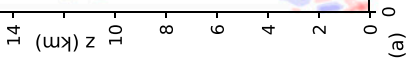

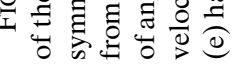



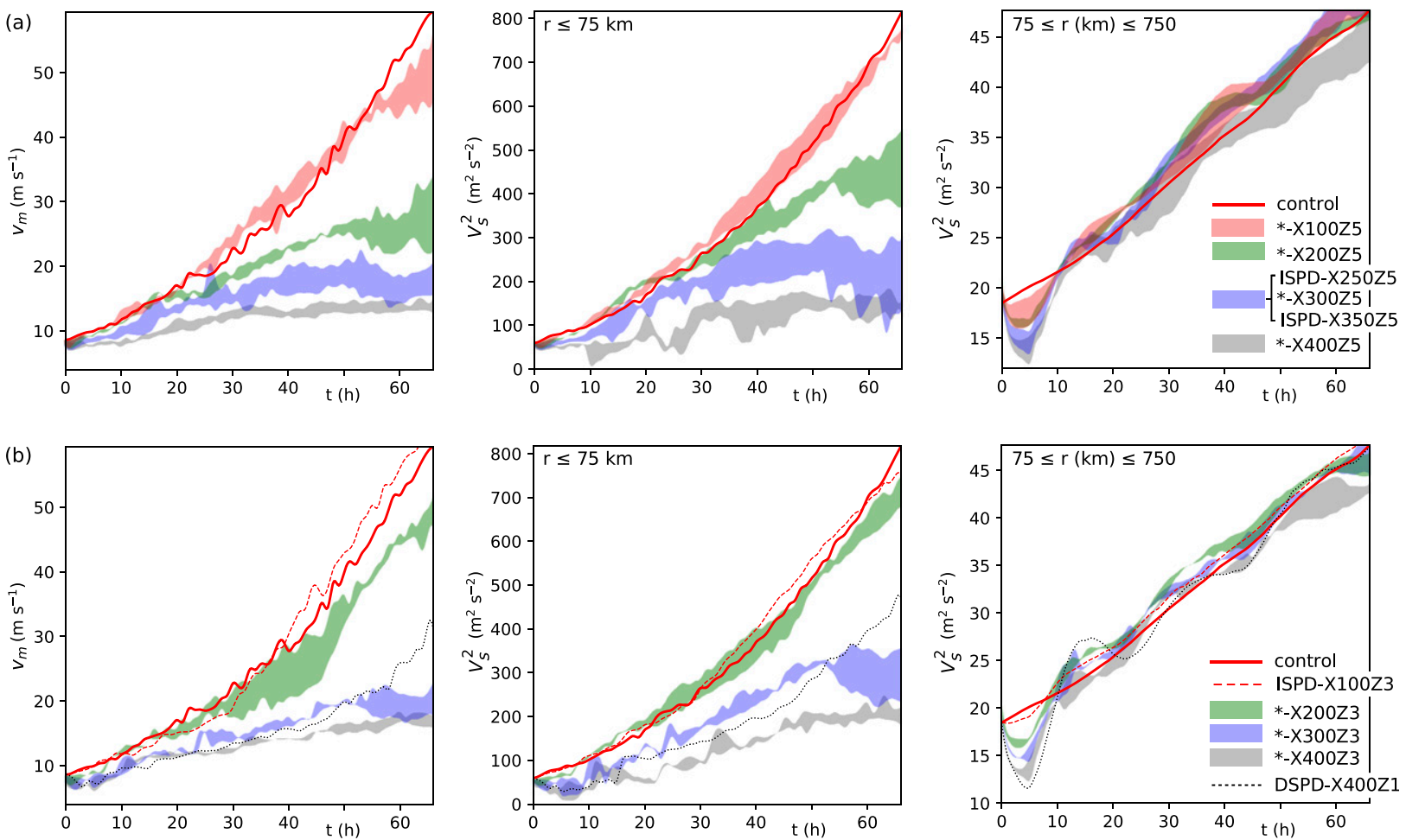

FIG. 13. (a) Time series of the (left) maximum and (center, right) mean-squared values of the azimuthally averaged tangential velocity field (in the SVC coordinate system) at the sea surface for several groups of simulations in which the misalignment is created by splitting the vortex at $z_{l}=5.25 \mathrm{~km}$. The radial averaging intervals for $V_{s}^{2}$ are (center) $r \leq 75 \mathrm{~km}$ and (right) $75 \leq r \leq 750 \mathrm{~km}$. The asterisk in the legend stands for all prefixes (IS, ISPD, and DSPD) existing for the given suffix. The upper and lower boundaries of each thickened curve trace the maximum and minimum values of the dependent variable within the corresponding group. The initial tilt magnitudes (the maximum values of $\left|\Delta \mathbf{x}_{c}\right|$ for $t \leq 6 \mathrm{~h}$ ) associated with each group are $86 \pm 5 \mathrm{~km}$ (light red), $148 \pm 24 \mathrm{~km}$ (green), $222 \pm 44 \mathrm{~km}$ (blue), and $308 \pm 42 \mathrm{~km}$ (gray); each of the preceding values is given as the group average plus or minus the standard deviation. The solid dark-red curve corresponds to the control simulation in which the vortex is virtually aligned throughout its development. (b) As in (a), but for simulations with $z_{l}=3.5$ and $1.75 \mathrm{~km}$. The initial tilt magnitudes are $68 \mathrm{~km}$ (dashed red), $156 \pm 13 \mathrm{~km}$ (green), $215 \pm 32 \mathrm{~km}(\mathrm{blue}), 285 \pm$ $41 \mathrm{~km}$ (gray), and $166 \mathrm{~km}$ (dotted black).

many (but not all) of the possibilities. Some of the known similarities and differences with other simulations will be noted as the narrative proceeds.

Before discussing the intricacies of tilt evolution in DSPD-X400Z5, it is worthwhile to briefly consider the potential relevance of ambient wind shear that may arise over time despite our elimination of the extraneous forcing that created the initial misalignment. Figure 15 shows time series of the magnitude and angle of the ambient shear vector, defined by $\left\langle\mathbf{u}_{m}-\mathbf{u}_{s}\right\rangle_{x y}$, in which $\mathbf{u}_{m}\left(\mathbf{u}_{s}\right)$ is the vertically averaged horizontal velocity in the middle-tropospheric (near-surface) layer corresponding to where $\mathbf{x}_{c m}\left(\mathbf{x}_{c s}\right)$ is measured. It is found that the shear magnitude remains weak $\left(0-0.35 \mathrm{~m} \mathrm{~s}^{-1}\right)$ and undulates over the course of the simulation (Fig. 15a). Ambient wind shear possessing one-half the maximum intensity seen here-acting in a direction parallel (antiparallel) to the tilt vector-would amplify (diminish) the tilt of the tropical cyclone at a rate of $15 \mathrm{~km} \mathrm{day}^{-1}$.
Such a rate is too small to account for the tilt tendencies found at any stage of evolution. Moreover, during the final and slowest stage of alignment, the shear vector rotates anticyclonically on a time scale that is short (an inertial period) compared to the precession period of the tilt vector (Fig. 15b). It follows that extrinsic forcing by ambient shear is not only weak but inefficient.

\section{a. Stage 1}

The first stage of tilt evolution is characterized by rapid decay of the measured misalignment. Such decay commonly coincides with the migration of $\mathbf{x}_{c s}$ toward an area of vigorous deep cumulus convection in the general direction of $\mathbf{x}_{c m}$. Figure 16 provides a minimal depiction of the process during the first $8 \mathrm{~h}$ of simulation DSPD$\mathrm{X} 400 \mathrm{Z} 5$. At any arbitrary instant during this 8 -h period, the surface center of rotation sits roughly in the middle of a $100-\mathrm{km}$ scale patch of cyclonic vorticity. Figure 16a 

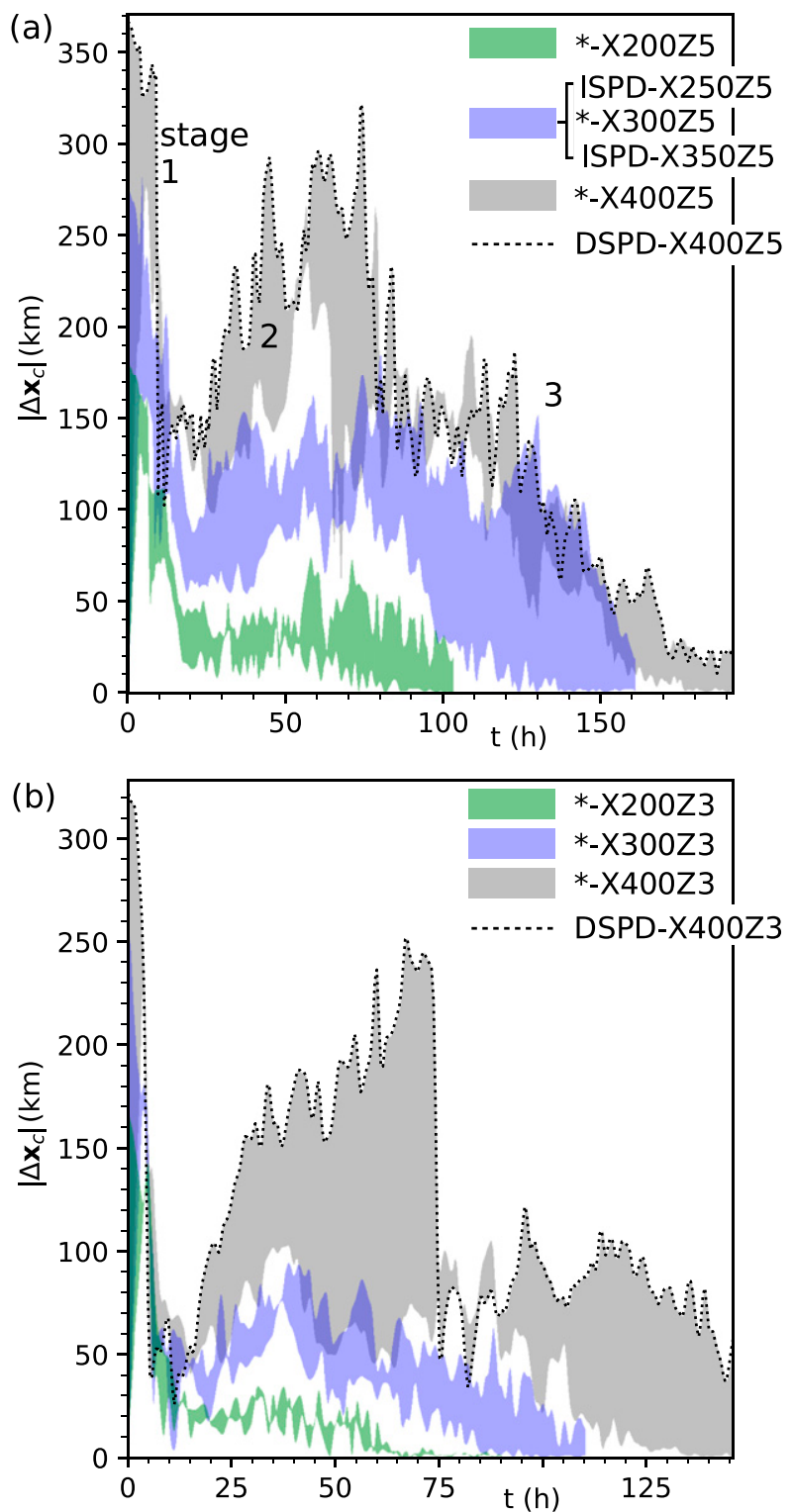

FIG. 14. (a) Time series of the tilt magnitude for several groups of simulations in which the misalignment is created by splitting the vortex at $z_{l}=5.25 \mathrm{~km}$. As in Fig. 13, the asterisk in the legend stands for all prefixes (IS, ISPD, and DSPD) among those existing for the given suffix. The dotted black curve belongs to the member of the gray group having the greatest tilt. (b) As in (a), but for $z_{l}=$ $3.5 \mathrm{~km}$. Note that each group in (b) has only two members.

shows the configuration at $t=6 \mathrm{~h}$. Figure 16a also illustrates how the aforementioned vorticity patch is exposed to irrotational winds that converge toward an (initially) outward moving band of convection. The irrotational velocity field $\mathbf{u}_{\chi}$ has a magnitude of approximately $2 \mathrm{~m} \mathrm{~s}^{-1}$ in the vicinity of $\mathbf{x}_{c s}$. One might therefore hypothesize that advection of the central vorticity patch by westerly irrotational winds has a nonnegligible role in

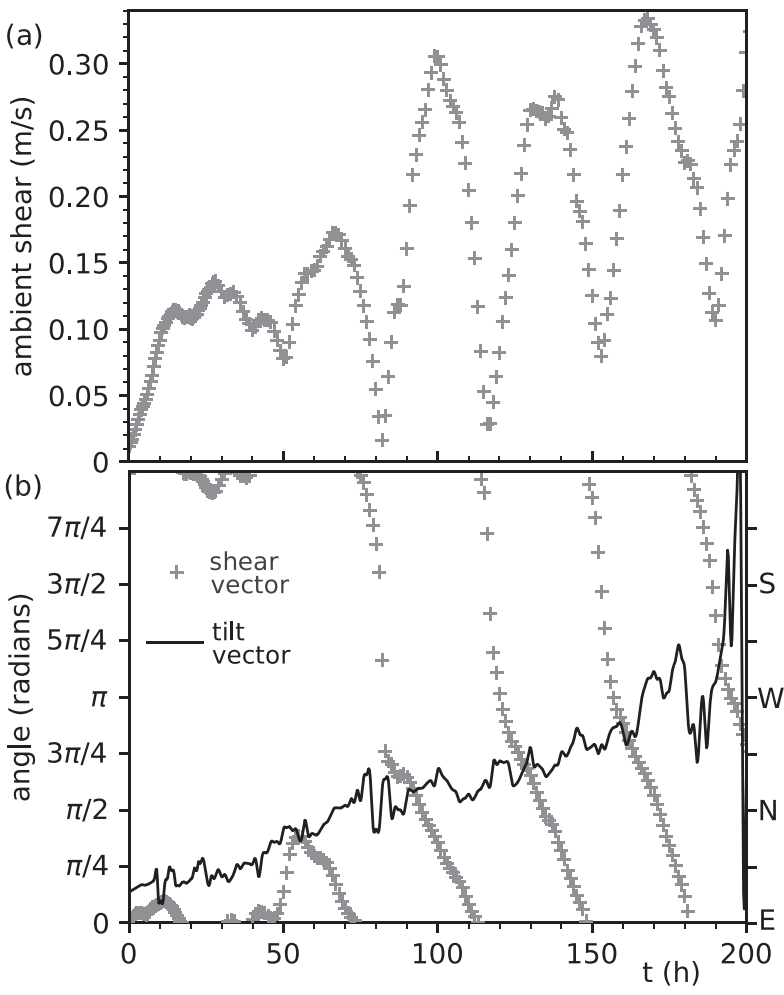

FIG. 15. (a) Magnitude of the ambient shear vector in simulation DSPD-X400Z5. (b) Angles of the ambient shear vector and tilt vector relative to the eastward direction.

the 75-km eastward drift of $\mathbf{x}_{c S}$ over an 8-h period. On the other hand, advection (by any part of $\mathbf{u}$ ) is not the entire story. Downtilt convection also reshapes and rescales the nondivergent (rotational) velocity field of the surface vortex that determines the location of $\mathbf{x}_{c s}$ (Fig. 16b). The nondivergent winds become enhanced in the east relative to the west. Furthermore, the radius of maximum surface wind speed $r_{m}$ increases from 52.5 to $70 \mathrm{~km}(131.25 \mathrm{~km})$ over the first $8 \mathrm{~h}(16 \mathrm{~h})$ of development. Thus, during its eastward migration, $\mathbf{x}_{c s}$ transitions from representing the center of a modest meso- $\beta$-scale vortex core to representing the center of a core that is 2-3 times larger.

Whereas the early convection-seeking drift of $\mathbf{x}_{c s}$ substantially reduces tilt in a number of other simulations, northeastward drift of $\mathbf{x}_{c m}$ largely counters such an effect in DSPD-X400Z5 (not shown). Instead, rapid reduction of tilt occurs through a sudden jump of the middle-tropospheric center of rotation to the area of deep convection (Fig. 17a). The jump apparently results from the emergence of intense middle-tropospheric vorticity anomalies within the updraft region of the mesoscale convective system (MCS; see Fig. 17c). Appendix B (Fig. B1b) illustrates the relatively strong rotational winds associated with the emergent disturbance. 

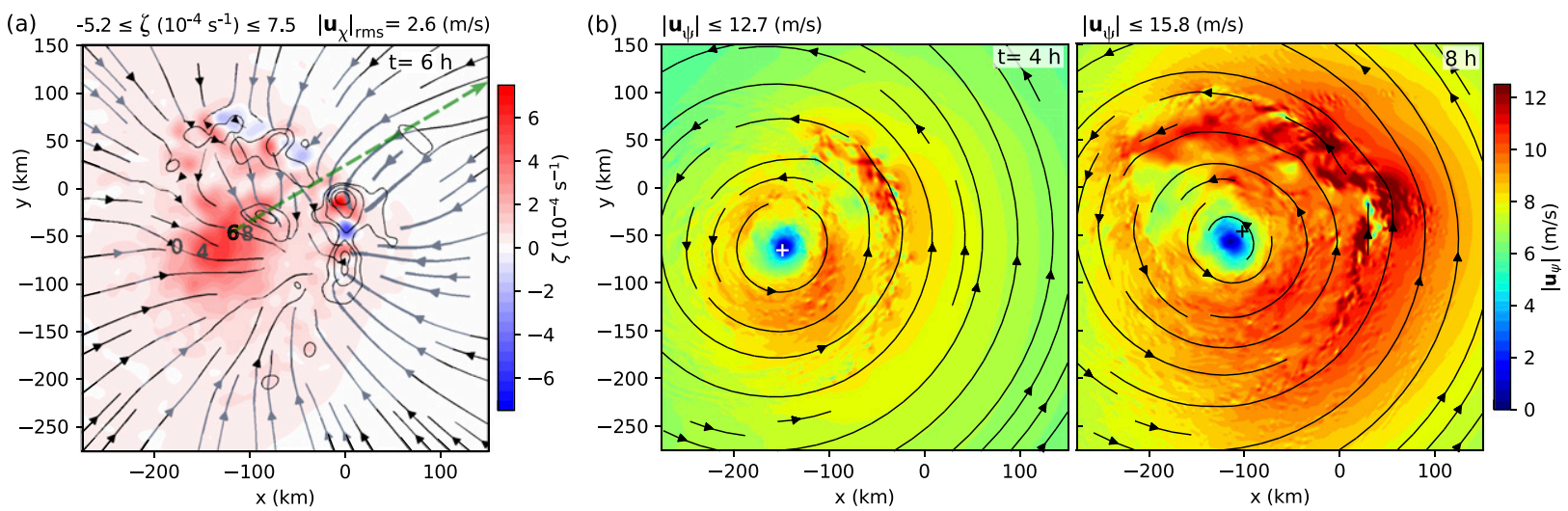

FIG. 16. Selected images of the boundary layer flow of simulation DSPD-X400Z5 in the fixed domain-centered coordinate system during the initial migration of $\mathbf{x}_{c s}$ toward vigorous convection downtilt. (a) Relative vertical vorticity $\zeta$ (color) and streamlines of the irrotational velocity field $\mathbf{u}_{\chi}$ in the boundary layer at $t=6 \mathrm{~h}$. Streamline thickness is proportional to the local irrotational wind speed; gray (black) indicates wind speeds greater than (less than) $2.0 \mathrm{~m} \mathrm{~s}^{-1}$. Thin black arrowless contours of $w$ at $z=8.9 \mathrm{~km}$ are superimposed on the plot to show where deep convection occurs at the time of the snapshot; the outermost-to-innermost contour levels are $0.2,1,3$, and $5 \mathrm{~m} \mathrm{~s}^{-1}$. The dashed green ray points in the direction of the tilt vector. The "rms" statistic above the top-right corner of the plot corresponds to the rootmean-square value of $\left|\mathbf{u}_{\chi}\right|$ in the depicted subregion of the simulation domain. The integers printed inside the plot show the location of $\mathbf{x}_{c s}$ at $t=$ $0,4,6$, and $8 \mathrm{~h}$, with the present location in black and all others in gray. (b) Streamlines and magnitude (color) of the nondivergent velocity field $\mathbf{u}_{\psi}$ in the boundary layer at (left) $t=4 \mathrm{~h}$ and (right) $t=8 \mathrm{~h}$; here the streamlines are given uniform thickness and shading. The plus sign shows the instantaneous location of $\mathbf{x}_{c s}$. All fields but $w$ in (a) and (b) are vertical averages between $z=0.025$ and $1.01 \mathrm{~km}$. The $\zeta$ and $w$ fields in (a) are smoothed in $x$ and $y$ using a Gaussian kernel with a standard deviation of $6.25 \mathrm{~km}$. See appendix C for the precise definitions of $\mathbf{u}_{\chi}$ and $\mathbf{u}_{\psi}$.

The sources of the vorticity anomalies are analyzed in appendix E. Notable vorticity anomalies are also found in the northern sector of the boundary layer of the MCS (Fig. 17b), but they are insufficiently strong in the aggregate to abruptly relocate $\mathbf{x}_{c s}{ }^{6}$

\section{b. Stage 2}

The subsequent regrowth of tilt in experiment DSPD-X400Z5 occurs gradually over a 2-day period of modest intensification of $v_{m}$. The first issue we address is whether moisture has an essential role in the process. Figure 18a compares the growth of $\left|\Delta \mathbf{x}_{c}\right|$ to that found in two dry adiabatic restarts at $t=28 \mathrm{~h}$. Two methods are used for restarting the model to demonstrate that details have little consequence on the result. The first restart eliminates cloud microphysics, dissipative heating and the surface enthalpy flux without any additional modifications. The second restart also reduces

\footnotetext{
${ }^{6}$ Substantial jumps of the middle-tropospheric vortex centers are common during stage 1 of the DSPD experiments that are included in Fig. 14. The same cannot be said for the IS and ISPD experiments, where $\mathbf{x}_{c m}$ generally starts in the vicinity of vigorous convection after the 6-h misalignment forcing ends. Recall that the IS and ISPD experiments are distinguished from their DSPD counterparts in permitting moist convection during the initial misalignment process. A period of rapid alignment nevertheless ensues in the IS and ISPD experiments as $\mathbf{x}_{c s}$ moves quickly toward $\mathbf{x}_{c m}$.
}

$C_{d}$ to $2.5 \times 10^{-5}$ and refines the fluid variables. The velocity field is refined by zeroing $w$, the irrotational component of $\mathbf{u}$, and the $z$-dependent horizontal mean of $\mathbf{u}$. The nondivergent component of $\mathbf{u}$ is obtained by inverting $\zeta$, adjusted to have zero vertical gradient in a 303-m layer adjacent to the sea surface. The final refinement involves enforcing conditions of nonlinear balance on $\theta$ and the pressure field (see appendix C). Both restarts demonstrate that the system would have a propensity to increase tilt under dry adiabatic dynamics somewhat faster and more effectively than the actual process occurs amid moist convection.

Figure 18b compares the moist and dry trajectories of $\mathbf{x}_{c s}$ and $\mathbf{x}_{c m}$. The middle-tropospheric vortex centers of the dry CM1 simulations move northward with their moist counterpart, but drift farther to the west. Early on, the surface center of the moist system is strongly inhibited from following any dry inclination to move southwest. Such inhibition is consistent with the common attraction of surface centers toward areas of vigorous deep convection, here situated to the northeast of $\mathbf{x}_{c s}$. Figure $18 \mathrm{~b}$ also shows the trajectories predicted by ideal 2D fluid dynamics. The 2D results come from two separate vortex-in-cell simulations (Leonard 1980) initialized with $\zeta$ distributions obtained from the pertinent surface and middle-tropospheric layers of the atmosphere at $t=28 \mathrm{~h}$. Each vortex-in-cell simulation has roughly $10^{8}$ vorticity elements, a rectangular mesh with $0.65-\mathrm{km}$ grid spacing, and doubly 

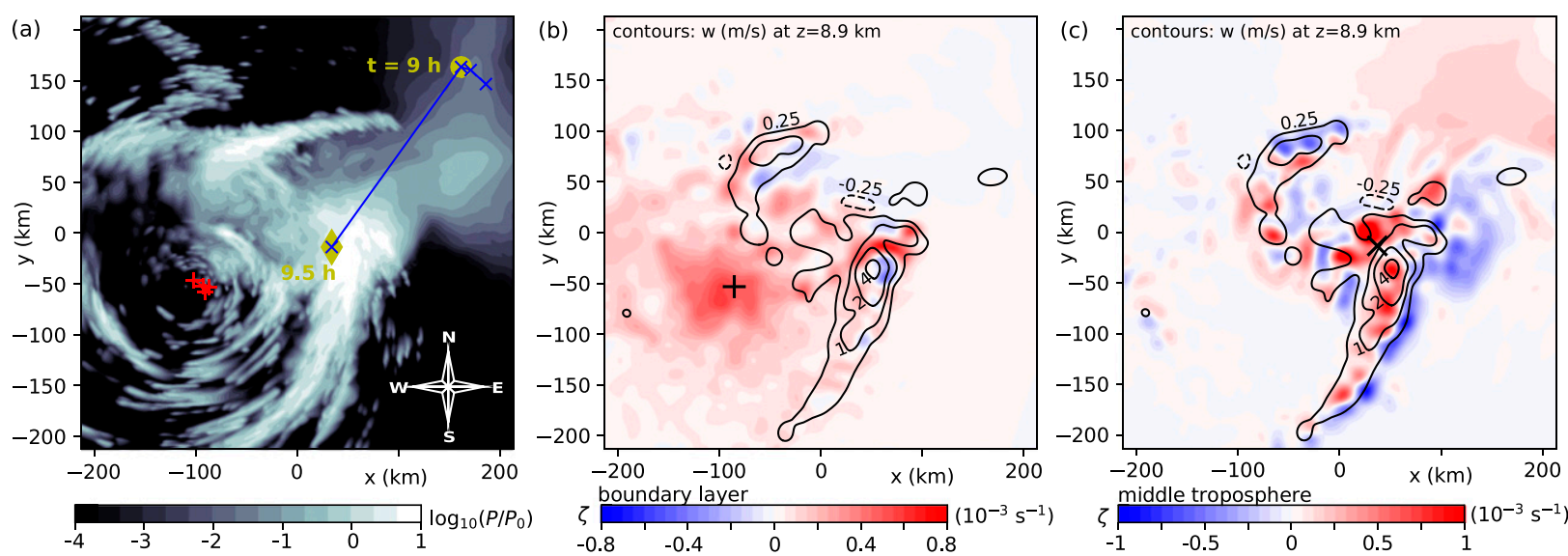

FIG. 17. Selected images associated with the abrupt reduction of tilt during stage 1 of simulation DSPD-X400Z5. (a) Surface and middletropospheric centers of rotation $\left[\mathbf{x}_{c s}(\mathrm{red}+)\right.$ and $\mathbf{x}_{c m}($ blue $\left.\times)\right]$ plotted every 30 min over the interval $8.0 \leq t \leq 9.5 \mathrm{~h}$. The center markers are superimposed on a plot of the logarithm of the accumulated precipitation $P$ (normalized to $P_{0}=1 \mathrm{~cm}$ ) during the aforementioned 1.5-h time interval. Yellow symbols highlight $\mathbf{x}_{c m}$ immediately before (circle) and after (diamond) it jumps to an area of enhanced precipitation. (b),(c) Vertical averages of the relative vertical vorticity distribution $\zeta$ immediately after the rapid reduction of tilt $(t=9.5 \mathrm{~h})$ in (b) the 1 $\mathrm{km}$ surface boundary layer and (c) the middle-tropospheric layer between $z=7.34$ and $8.13 \mathrm{~km}$. A contour plot of the vertical velocity field $w$ at $z=8.9 \mathrm{~km}$ (averaged over $8 \leq t \leq 9.5 \mathrm{~h}$ ) is superimposed on each vorticity distribution. Both $\zeta$ and $w$ are smoothed in the horizontal plane using a Gaussian kernel with a standard deviation of $6.25 \mathrm{~km}$ in both dimensions. The + and $\times$ in (b) and (c) respectively mark $\mathbf{x}_{c s}$ and $\mathbf{x}_{c m}$ at the time of the vorticity snapshot. The origin of the coordinate system in each plot corresponds to the fixed center of the simulation domain.

periodic boundary conditions equivalent to those of the CM1 simulations. The middle-tropospheric trajectory predicted by 2D dynamics remains relatively close to that of the moist system. Such closeness may be somewhat coincidental, but reproduction of the basic northward drift suggests some relevance of the $2 \mathrm{D}$ model. By contrast, the $2 \mathrm{D}$ surface trajectory strays considerably from its moist counterpart, and ends up far west of all 3D systems.

Figures $18 \mathrm{c}$ and $18 \mathrm{~d}$ show snapshots of the moist middle-tropospheric vortex near the start and end of the northward drift of $\mathbf{x}_{c m}$ that is largely responsible for the regrowth of tilt. It is seen that the drift coincides with considerable reshaping of an asymmetric vertical vorticity distribution with multiscale structure and a prominent band extending outward from the core. The process occurs amid continual 3D-adiabatic and diabatic perturbations of $\zeta$. The associated irrotational winds represented by $\mathbf{u}_{\chi}$ are nontrivial (Fig. 18c), but as for any predominantly vortical flow, the nondivergent component of the velocity field $\mathbf{u}_{\psi}$ is characteristically stronger (Fig. 18d). The root-meansquare (rms) value of $\left|\mathbf{u}_{\psi}\right|$ is 3.4 times the rms value of $\left|\mathbf{u}_{\chi}\right|$ over the depicted area in both snapshots. ${ }^{7}$

The relative strength of $\mathbf{u}_{\psi}$ combined with the qualitatively successful prediction of the vortex-in-cell simulation

\footnotetext{
${ }^{7}$ The domain-averaged velocity $\mathbf{u}_{a}$-the third component of the Helmholtz decomposition (appendix C) viewed in the ES reference frame-has a negligible magnitude of $0.1 \mathrm{~m} \mathrm{~s}^{-1}$ in the layer containing $\mathbf{x}_{c m}$.
}

in the middle troposphere motivate further consideration of how nondivergent 2D dynamics may contribute to the drift of $\mathbf{x}_{c m}$. Figure 19 splits the middle-tropospheric nondivergent velocity field into two parts during the northward drift period. The first part $\mathbf{u}_{w}^{c}$ is obtained by inverting the unfiltered vertical vorticity distribution inside the closed black curve in Fig. 19a (at $t=31 \mathrm{~h}$ ) or Fig. 19d (at $t=44 \mathrm{~h}$ ) using a free-space Green function. The aforementioned curve is essentially a contour where $\zeta=5 \times 10^{-6} \mathrm{~s}^{-1}$ after Gaussian smoothing with a kernel whose decay length is $30 \mathrm{~km}$ in both horizontal dimensions. By design, $\mathbf{u}_{w}^{c}$ (Figs. 19b,e) represents the nondivergent winds of the predominantly cyclonic core of the middle-tropospheric vortex. The second part is defined by $\mathbf{u}_{\psi}^{e} \equiv \mathbf{u}_{\psi}-\mathbf{u}_{\psi}^{c}$ (Figs. 19c,f) and represents the nondivergent winds of structures external to the core. ${ }^{8}$ It is seen that negative vorticity to the east generates an anticyclonic gyre in $\mathbf{u}_{w}^{e}$ that alone would nudge the bulk of the cyclonic core toward the north (northeast). ${ }^{9}$ Such nudging does not seem incidental to the drift of $\mathbf{x}_{c m}$, but

\footnotetext{
${ }^{8}$ As such, $\mathbf{u}_{\psi}^{e}$ includes minor contributions from images of the core associated with periodic boundary conditions.

${ }^{9} \mathrm{We}$ have verified that similar scenarios unfold in the middle troposphere during the regrowth of the misalignments in ISX400Z5 and ISPD-X400Z5, which have comparable initial tilt magnitudes. Thus, the eastern anticyclonic vorticity patch does not appear to be a unique consequence of the DSPD method for generating the initial misalignment.
} 

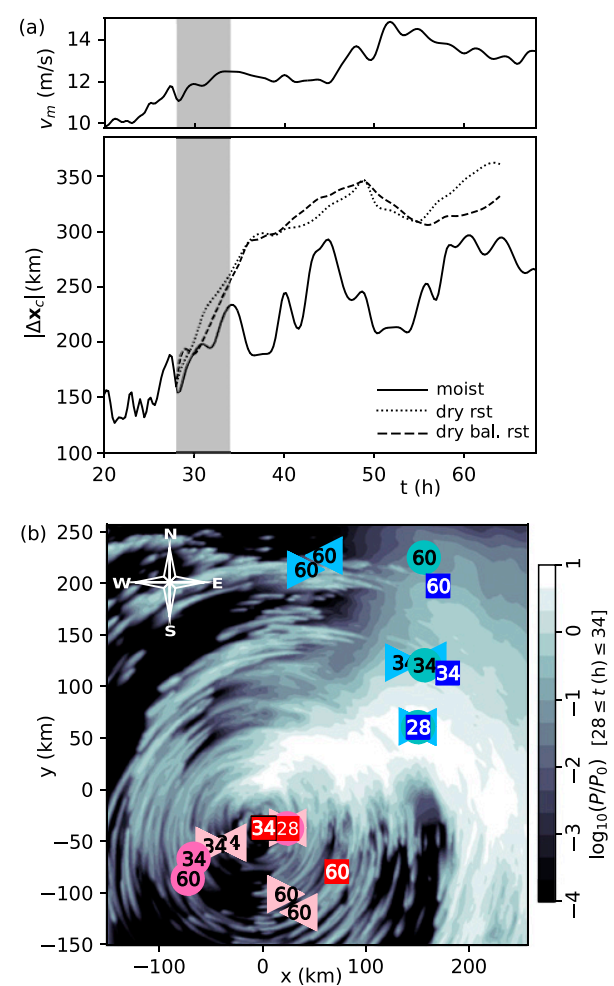
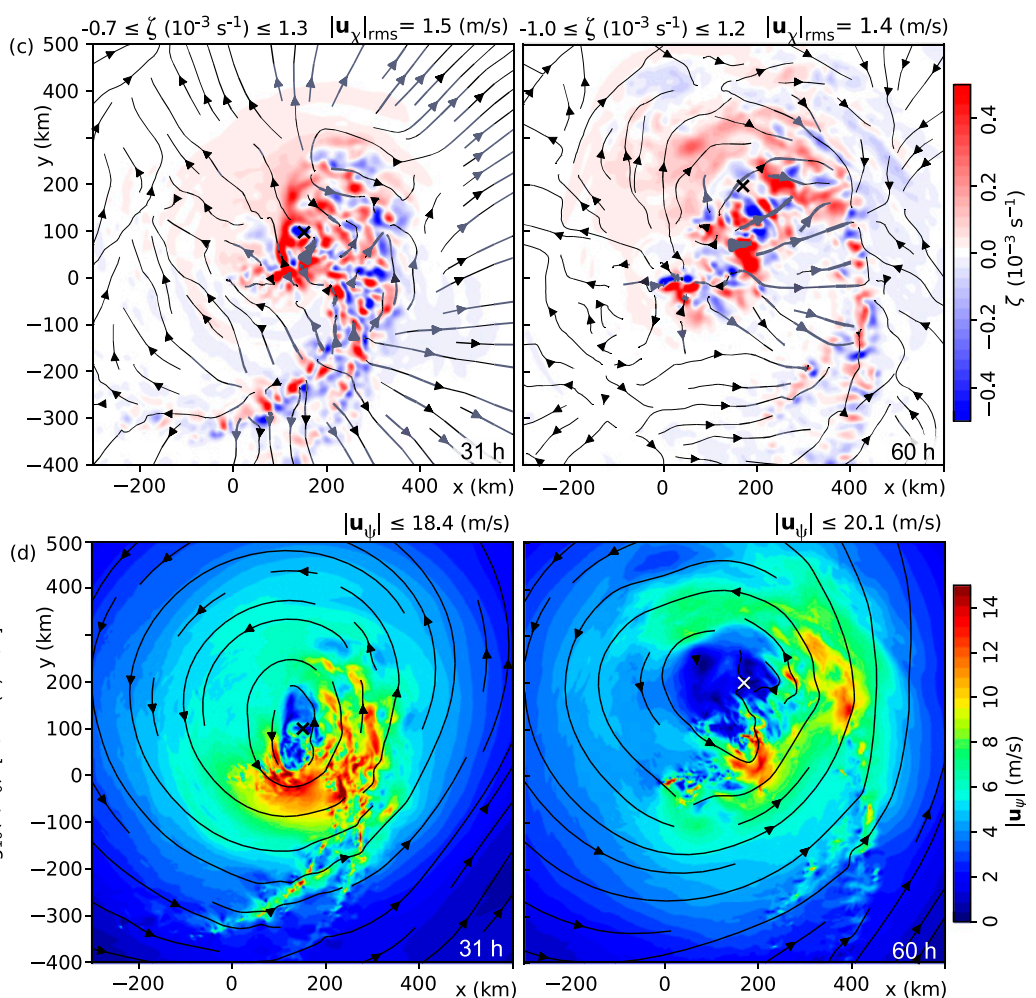

FIG. 18. Stage 2 of tilt evolution in experiment DSPD-X400Z5. (a) (bottom) Time series of the tilt magnitudes in the moist CM1 simulation, an unrefined dry restart and a dry balanced restart. (top) Attendant growth of $v_{m}$ in the moist simulation. (b) Surface and middle-tropospheric centers of rotation at $t=28,34$, and $60 \mathrm{~h}$. Squares show $\mathbf{x}_{c s}$ (red) and $\mathbf{x}_{c m}$ (blue) from the moist simulation. Triangles show $\mathbf{x}_{c s}$ (light pink) and $\mathbf{x}_{c m}$ (light blue) from the unrefined dry restart (left pointing) and the dry balanced restart (right pointing) initialized at $t=28 \mathrm{~h}$. Circles show $\mathbf{x}_{c s}$ (dark pink) and $\mathbf{x}_{c m}$ (cyan) predicted by 2D vortex-in-cell simulations of the boundary layer and middle-tropospheric layer starting at $t=28 \mathrm{~h}$. The position markers are superimposed on a plot of the logarithm of the accumulated precipitation $P$ (normalized to $P_{0}=1 \mathrm{~cm}$ ) between $t=28$ and $34 \mathrm{~h}$. (c) Relative vertical vorticity $\zeta$ (color) and streamlines of $\mathbf{u}_{\chi}$ in the middle-tropospheric layer containing $\mathbf{x}_{c m}(\times)$ in the moist simulation at (left) $t=31 \mathrm{~h}$ and (right) $t=60 \mathrm{~h}$. Vorticity is smoothed in the horizontal plane using a Gaussian kernel with a standard deviation of $6.25 \mathrm{~km}$ in both dimensions. Streamline thickness is proportional to $\left|\mathbf{u}_{\chi}\right|$; gray (black) indicates irrotational wind speeds greater than (less than) $1.5 \mathrm{~m} \mathrm{~s}^{-1}$. (d) Corresponding snapshots of the streamlines and magnitude (color) of $\mathbf{u}_{\psi}$; here the streamlines are given uniform thickness and shading. All plotted fields in (c) and (d) are vertical averages over the interval $7.34 \leq z \leq 8.13 \mathrm{~km}$. The origin of the coordinate system in (b)-(d) is located at the fixed center of the simulation domain.

one should bear in mind that more subtle $2 \mathrm{D}$ or $3 \mathrm{D}$ mechanisms unapparent from the preceding analysis could have equal or even greater importance.

Although regrowth of the tilt magnitude in a cloudresolving simulation of tropical cyclone development without assistance from environmental wind shear may seem surprising, the spontaneous misalignment of a vortex is not an entirely novel phenomenon. Previous discussions of spontaneous misalignment have often dealt with tilts arising from essentially adiabatic, threedimensional circular shear flow instabilities (Gent and McWilliams 1986; Schecter et al. 2002; Reasor et al. 2004). Jones (2000a) examined what might be viewed as the nonlinear stage of an instability contributing to the growth of tilt in a dry vortex simultaneously interacting with environmental winds. The associated dynamics happened to resemble that described above, in which an outer anticyclonic vorticity anomaly acts to drive the cyclonic core of the vortex in one layer of the atmosphere away from the core in another layer. While the similarity is intriguing, we caution against extending the analogy too far. Events leading up to the foregoing scenario in DSPD-X400Z5 are distinct in part by having a substantial diabatic element (see section $4 \mathrm{a}$ and appendix E). There is also evidence, provided below, suggesting that convection and its associated irrotational winds significantly modulate the drift of $\mathbf{x}_{c m}$ during the tilt amplification period.

The aforementioned evidence is found by viewing the drift of the middle-tropospheric cyclone from a perspective that is more directly connected to the measurement of $\mathbf{x}_{c m}$. Recall that $\mathbf{x}_{c}$ is essentially the point in a specified layer where the centering of a polar coordinate system yields the largest peak value of $\bar{v}_{\psi}(=\bar{v})$. Any drift 

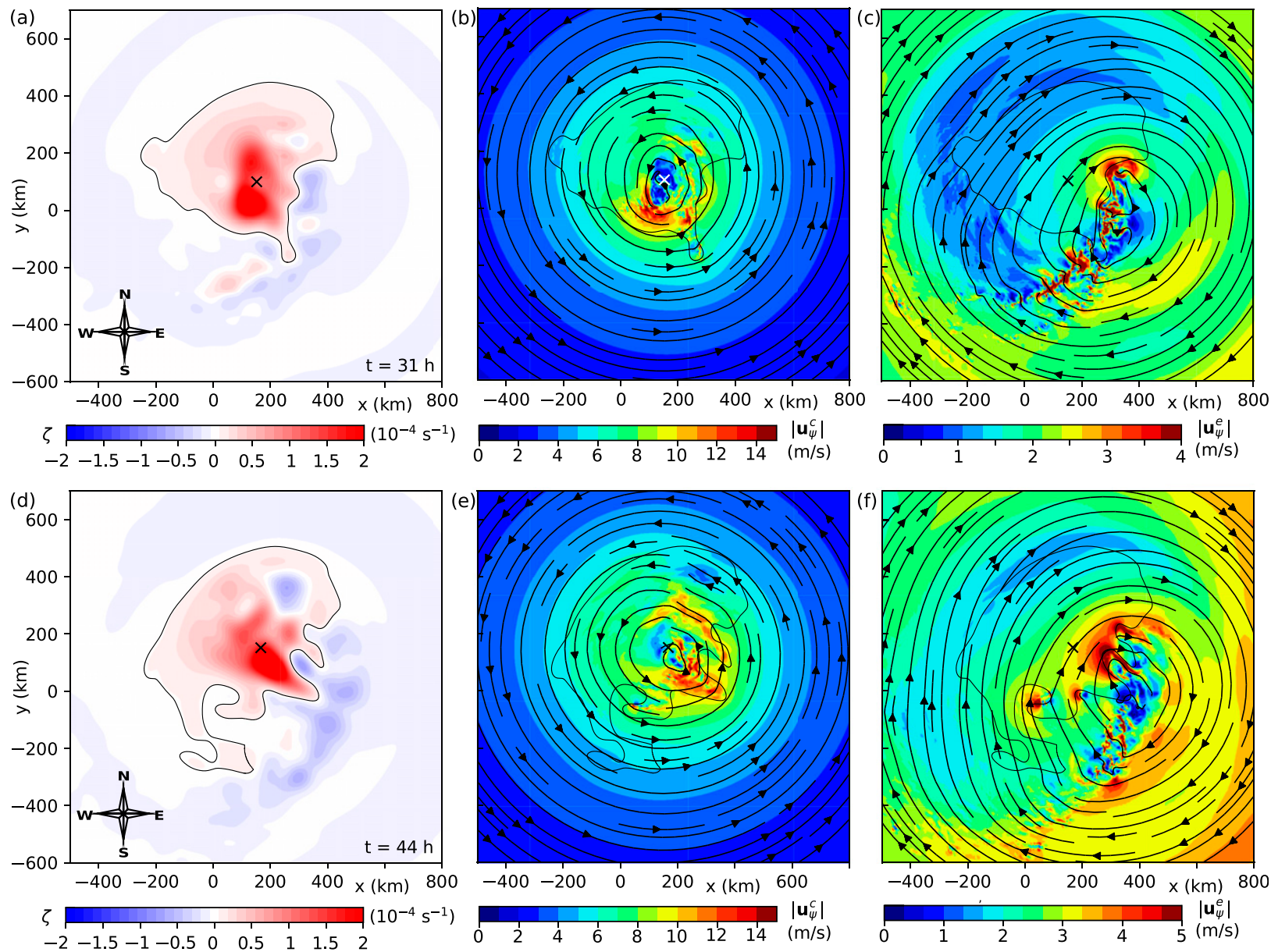

FIG. 19. (a)-(c) Decomposition of the nondivergent winds in the middle troposphere near the beginning of the tilt restoration period $(t=31 \mathrm{~h})$ in simulation DSPD-X400Z5. (a) Relative vertical vorticity smoothed using a Gaussian kernel with a standard deviation of $30 \mathrm{~km}$ in both $x$ and $y$. The closed black curve is a contour where the smoothed vorticity distribution equals $5 \times 10^{-6} \mathrm{~s}^{-1}$. The predominantly red area enclosed by this contour contains the (unsmoothed) vorticity that generates $\mathbf{u}_{\psi}^{c}$. (b),(c) Streamlines and magnitude of (b) the core component $\mathbf{u}_{w}^{c}$ and (c) the external component $\mathbf{u}_{w}^{e}$ of the nondivergent velocity field. The thin black contour without arrows is equivalent to that in (a). All plotted fields are vertical averages over the interval $7.34 \leq z \leq 8.13 \mathrm{~km}$. The location of $\mathbf{x}_{c m}$ is marked by an $\times$. (d)-(f) As in (a)-(c), but for $t=44 \mathrm{~h}$.

entails a change of this point over time, which under general circumstances cannot be understood as a purely advective process. Consider the time interval between hours 28 and 34 of the moist simulation. The top panels in Figs. 20a and 20b show the middle-tropospheric distributions of $\bar{v}$ (vertical averages over $7.34 \leq z \leq 8.13 \mathrm{~km}$ ) at the start and end of the 6-h analysis period in two stationary coordinate systems. One coordinate system $\left(\mathrm{CS}_{i}\right)$ is centered on the initial location of $\mathbf{x}_{c m}$ (Fig. 20b); the other $\left(\mathrm{CS}_{f}\right)$ is centered on the final location of $\mathbf{x}_{c m}$ (Fig. 20a). The top panels indicate that the drift is attributable to maintenance-as opposed to appreciable amplification - of mean-vortex intensity in $\mathrm{CS}_{f}$ in conjunction with decay of maximal $\bar{v}$ in $\mathrm{CS}_{i}$. The bottom panels of Figs. 20a and 20b show the $\bar{v}$ budgets (vertically averaged over the pertinent middle-tropospheric layer and time averaged over the analysis period) decomposed as explained in appendix $\mathrm{F}$. The contribution to $\partial_{t} \bar{v}$ from the radial influx of vertical vorticity driven by the nondivergent winds $\left(-\overline{u_{\psi} \zeta^{\prime}}\right)$ acts to intensify the mean vortex where $\bar{v}$ is peaked in $\mathrm{CS}_{f}$ (Fig. 20a), while it weakens the mean vortex where $\bar{v}$ is initially peaked in $\mathrm{CS}_{i}$ (Fig. 20b). The aforementioned intensification effort in $\mathrm{CS}_{f}$ is notably tempered by the net impact of the absolute vorticity influx driven by irrotational winds $\left[-\overline{u_{\chi} \eta} \equiv-\overline{u_{\chi}(\zeta+f)}\right]$, the vertical advection of angular momentum $\left(-\overline{w \partial_{z} v}\right)$, and the relatively minor forcing by subgrid turbulence $\left(\overline{\mathcal{T}}_{v}\right)$. The significance of budget terms other than $-\overline{u_{\psi} \zeta^{\prime}}$ is consistent with moderate deviation of $\mathbf{x}_{c m}$ from the ideal 2D trajectory (Fig. 18b), and suggests that any drift that may arise from the nudging of the middle-tropospheric cyclone 

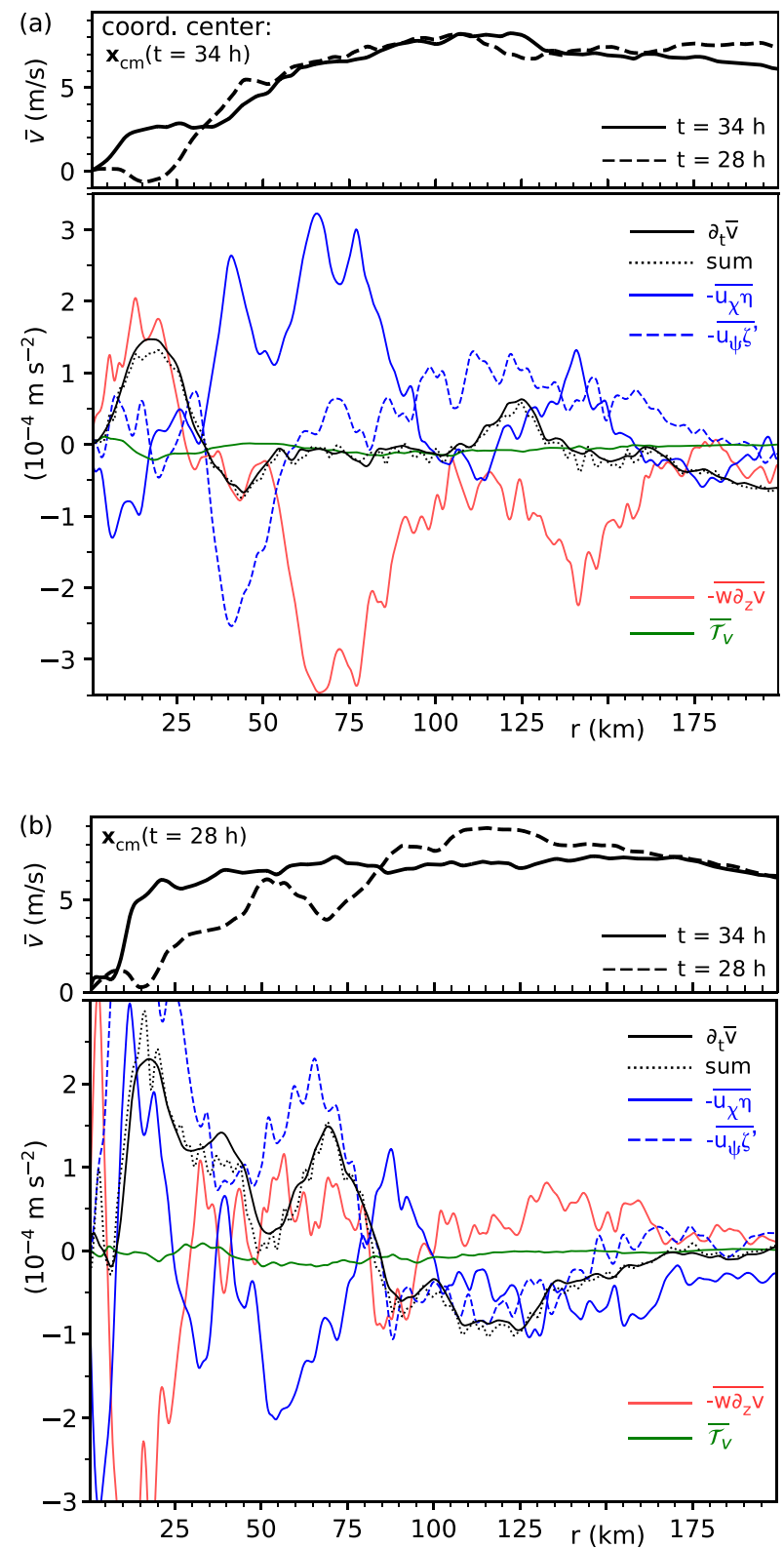

FIG. 20. Changes of middle-tropospheric $\bar{v}$ as seen from the end point and start point of the northward trajectory of $\mathbf{x}_{c m}$ in simulation DSPD-X400Z5 during the shaded time interval in Fig. 18a (28 $\leq t \leq 34 \mathrm{~h}$ ). (a) (top) Snapshots of $\bar{v}$ (averaged between $z=7.34$ and $8.13 \mathrm{~km})$ measured in a coordinate system $\left(\mathrm{CS}_{f}\right)$ centered where $\mathbf{x}_{c m}$ is located at the end of the analysis period. The solid (dashed) curve corresponds to $\bar{v}$ at the end (start) of the analysis period. (bottom) Tendency of layer-averaged $\bar{v}$ (solid black) and various contributions to that tendency averaged in $t$ over the 6-h analysis period. The contributions from $-\overline{u_{a} \zeta^{\prime}}$ and $-c_{\mathrm{pd}} \overline{\theta_{\rho}^{\prime} \partial_{\varphi} \Pi^{\prime}} / r$ are relatively small $\left(\ll 10^{-4} \mathrm{~m} \mathrm{~s}^{-2}\right)$ and excluded from the plot. The depicted "sum" (dotted black) includes these and all other (red, green, and blue) contributions. (b) As in (a), but for measurements in a coordinate system $\left(\mathrm{CS}_{i}\right)$ centered where $\mathbf{x}_{c m}$ is located at the start of the analysis period. by $\mathbf{u}_{\psi}^{e}$ (Figs. 19c,f) is at least modulated by other factors associated with convection. ${ }^{10}$

\section{c. Stage 3}

The final stage of tilt evolution brings the system to a state of virtual alignment. Dry adiabatic restarts cannot reproduce the sustained alignment trends found in the moist simulation (Fig. 21a). It stands to reason that diabatic cloud processes are essential. Figure $21 \mathrm{~b}$ shows the near-surface and middle-tropospheric vortex trajectories over the period between hours 165 and 171 of development, which essentially covers the final surge to an aligned state. The trajectories are superimposed over a depiction of the attendant moist convection. Whereas $\mathbf{x}_{c m}$ shifts little within the broader updraft region of a convective complex,${ }^{11} \mathbf{x}_{c s}$ darts westward toward its counterpart.

Figure 22 illustrates the nature of the fluid dynamics during the westward motion of $\mathbf{x}_{c s}$. The top row shows that the near-surface cyclone consists of multiple meso$\gamma$-scale vortices immersed in diffuse, predominantly cyclonic background vorticity. The meso- $\gamma$-scale vortices are typically products of convection in either the core of the parent cyclone or peripheral rainbands. Some may travel far away from their points of origin over the course of a lifetime; the three prominent eastern vortices at $t=165 \mathrm{~h}$ notably emerged from the main area of convection west of $\mathbf{x}_{c s}$. The irrotational velocity field consists of broad inflow from the outer part of the cyclone and zones of confluence near active convection. Relatively strong western confluence of $\mathbf{u}_{\chi}$ may contribute significantly to the westward shift of $\mathbf{x}_{c s}$. On the other hand, the rms wind speed of the nondivergent velocity field is 5.6 times that of $\mathbf{u}_{\chi}$ in each depicted snapshot of the evolving system. Generic mixing processes typical of nondivergent $2 \mathrm{D}$ flows seem to have a nontrivial role in reshaping the vorticity distribution. The bottom row of the figure shows the nondivergent velocity field and helps clarify why the measurement of $\mathbf{x}_{c s}$ moves westward. The intensity distribution becomes highly skewed to the west before the strongest winds become more evenly distributed about a $100-\mathrm{km}$ scale circle whose center is substantially displaced (to the west) from where the surface vortex center resided $6 \mathrm{~h}$ earlier.

\footnotetext{
${ }^{10}$ This provisional interpretation of the $\bar{v}$ budget assumes that $-\overline{u_{\chi} \eta}$ and $-\overline{w \partial_{z} v}$ are strongly influenced by convective processes, with the caveat that the preceding terms are not necessarily negligible in dry dynamics (e.g., Schecter 2017).

${ }^{11}$ We note that prior convective reorganization of the middletropospheric flow similar (in some ways) to that seen during stage 1 (Figs. 17 and B1b) played a major role in bringing $\mathbf{x}_{c m}$ to this location, following the regrowth of tilt during stage 2 .
} 

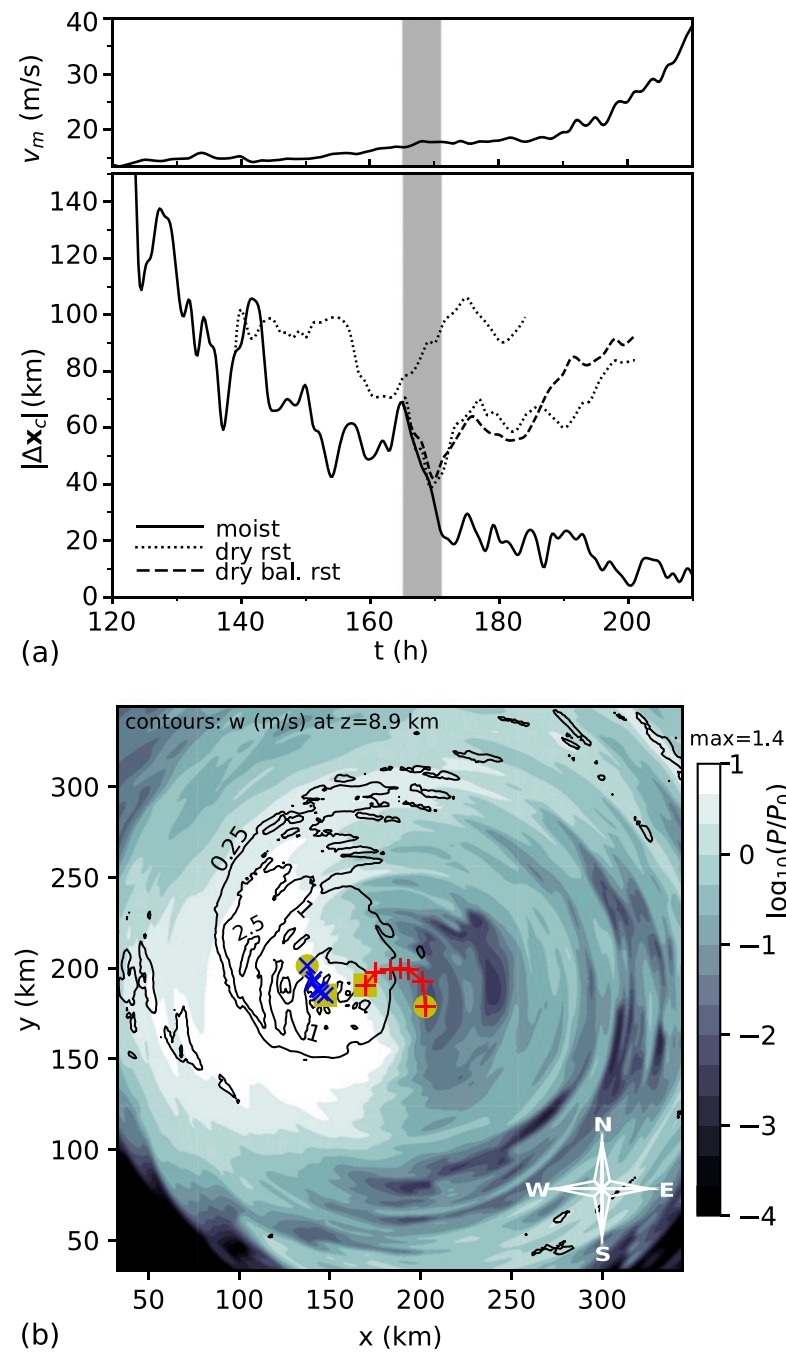

FIG. 21. Overview of stage 3 of tilt evolution in simulation DSPD-X400Z5. (a) (bottom) Time series of the tilt magnitude in the moist CM1 simulation (solid), two unrefined dry adiabatic restarts (dotted), and one dry adiabatic restart initialized in nonlinear balance (dashed). (top) Attendant growth of $v_{m}$ in the moist simulation. (b) Surface and middle-tropospheric centers of rotation $\left[\mathbf{x}_{c s}(\right.$ red +$)$ and $\mathbf{x}_{c m}($ blue $\times)$ ] plotted hourly over the shaded time interval (165 $\leq t \leq 171 \mathrm{~h})$ in (a). Yellow circles (squares) indicate the start time (end time). The background shading shows the logarithm of the accumulated precipitation $P$ (normalized to $P_{0}=1 \mathrm{~cm}$ ) over the aforementioned 6-h time interval. The contours show the corresponding 6-h time-averaged vertical velocity field at $z=8.9 \mathrm{~km}$. The origin of the coordinate system corresponds to the fixed center of the simulation domain.

To elaborate, the westward drift of $\mathbf{x}_{c s}$ coincides with the amplification of maximal $\bar{v}$ near the surface in a fixed coordinate system $\left(\mathrm{CS}_{f}\right)$ centered at the end point of the 6-h trajectory. Various contributions to the amplification can be seen in the azimuthal velocity budget observed in $\mathrm{CS}_{f}$. Figure 23a shows the budget with each term vertically averaged over the near-surface layer $(z \leq 1.01 \mathrm{~km})$ and temporally averaged over the $6-\mathrm{h}$ analysis period. The combined effect of the radial influx of absolute vorticity driven by the irrotational winds $\left(-\overline{u_{\chi} \eta}\right)$ and the vertical advection of angular momentum $\left(-\overline{w \partial_{z} v}\right)$ is consistently positive. The $\bar{v}$ tendency associated with parameterized turbulence $\left(\overline{\mathcal{T}}_{v}\right)$ is substantial and consistently negative, but the importance on the drift mechanism is unclear. It is found that $\overline{\mathcal{T}}_{v}$ becomes negligible slightly above the nearsurface layer (Fig. 23b) where the vorticity evolution and motion of $\mathbf{x}_{c}$ are very similar. Interestingly, both within and slightly above the near-surface layer, the radial influx of vorticity driven by nondivergent winds $\left(-\overline{u_{\psi} \zeta^{\prime}}\right)$ plays a decisive role in determining the positive growth of $\bar{v}$ where it becomes maximal. This does not imply that the influence of moist convection on the drift of $\mathbf{x}_{c s}$ is somehow nullified. Among other factors to consider, convection modifies both the vorticity perturbation and the coupled nondivergent winds. The importance of convection and $\mathbf{u}_{\chi}$ is less concealed when restricting the analysis to the first $3 \mathrm{~h}$ of the drift period [ $165 \leq t \leq 168 \mathrm{~h}$ ]. Figures $23 \mathrm{c}$ and $22 \mathrm{~d}$ show that in a fixed coordinate system centered where $\mathbf{x}_{c s}$ resides at $t=168 \mathrm{~h}$, the positive combination of $-\overline{w \partial_{z} v}$ and $-\overline{u_{\chi} \eta}$ is integral to the $3-\mathrm{h}$ amplification of $\bar{v}$ in the neighborhood of the radius of maximum wind.

As a final remark, the third stage of tilt evolution coincides with fairly slow growth of $v_{m}$ (Fig. 21a). The onset of rapid intensification does not occur until nearly one full day after the final surge of alignment that brings $\left|\Delta \mathbf{x}_{c}\right|$ down to approximately $20 \mathrm{~km}$.

\section{d. Comment on alignment paradigms based on vortex Rossby wave dynamics}

There exists a sizable body of literature on the potential importance of vortex Rossby (VR) wave dynamics in contributing to the alignment process (Reasor and Montgomery 2001,2015; Reasor et al. 2004; Schecter et al. 2002; Schecter and Montgomery 2003,2007; Schecter 2015). In quasi-balanced linear perturbation theory, a relatively weak tilt decomposes into a set of discrete and sheared VR waves. ${ }^{12}$ If stability conditions are satisfied, free alignment may occur by the outward propagation and spiral windup of sheared VR waves, or by the

\footnotetext{
${ }^{12}$ Here, the term "discrete VR wave" refers to a genuine discrete normal mode of the linear perturbation equations or a quasimode, which is more frequently the case in the present context.
} 

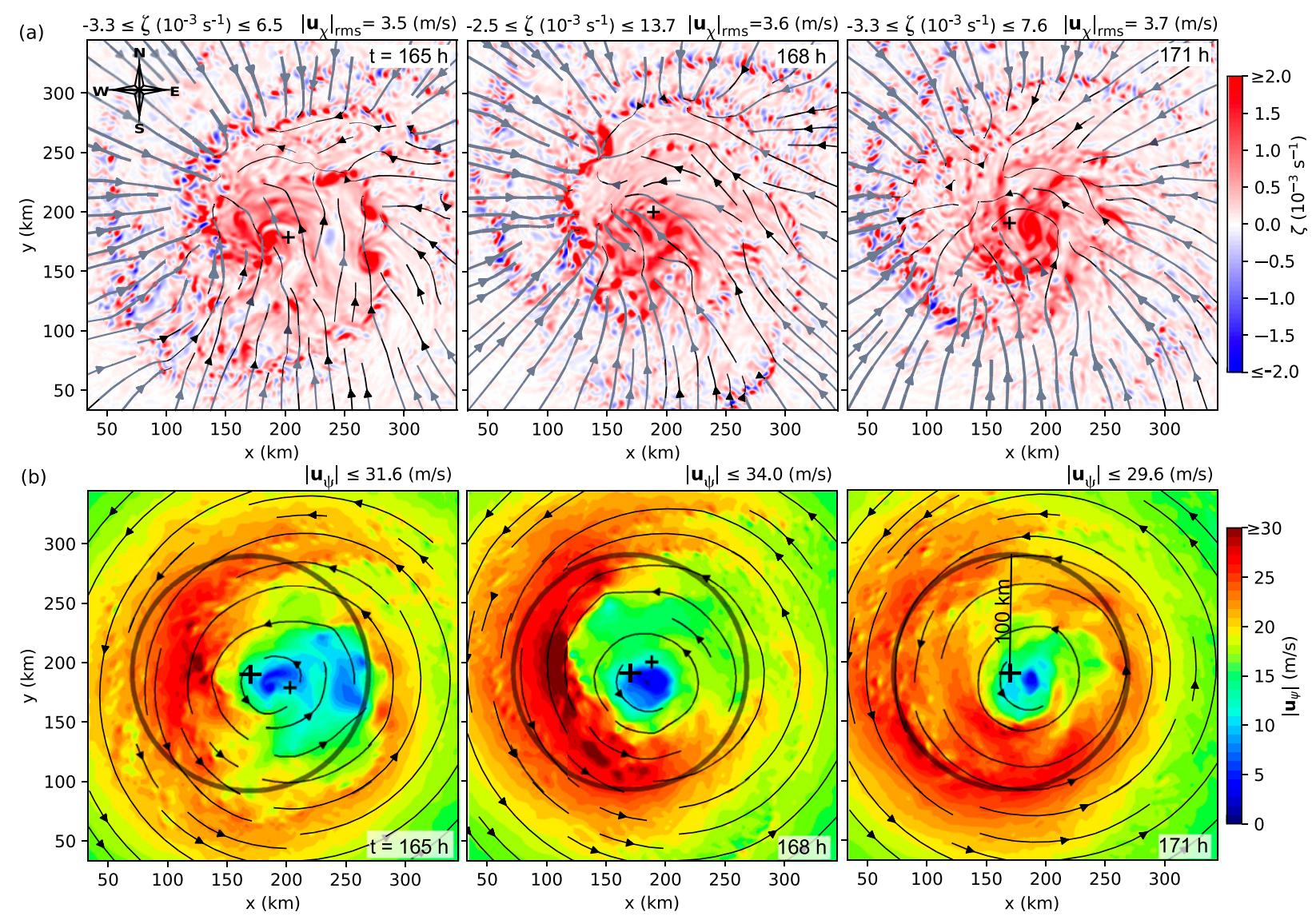

FIG. 22. Selected snapshots of the boundary layer flow of simulation DSPD-X400Z5 in the fixed domain-centered coordinate system during the final surge toward alignment (the shaded time interval in Fig. 21a). (a) Relative vertical vorticity $\zeta$ (color) and streamlines of the irrotational velocity field $\mathbf{u}_{\chi}$. Streamline thickness is proportional to the local irrotational wind speed; gray (black) indicates wind speeds greater than (less than) $2.5 \mathrm{~m} \mathrm{~s}^{-1}$. (b) Streamlines and magnitude (color) of the nondivergent velocity field $\mathbf{u}_{\psi}$; here the streamlines are given uniform thickness and shading. All fields in (a) and (b) are vertical averages over the interval $0.025 \leq z \leq 1.01 \mathrm{~km}$ but are unsmoothed in $x$ and $y$. The instantaneous location of $\mathbf{x}_{c s}$ is marked by a small + . The thick semitransparent gray circle in (b) is centered on the large + , which marks the location of $\mathbf{x}_{c s}$ at $t=171 \mathrm{~h}$ and serves as the fixed coordinate center for the analysis in Figs. 23a and 23b.

negative feedback that a discrete VR wave will receive upon exciting a potential vorticity (PV) perturbation in a critical layer.

A reasonable estimate of the time scale for outward propagation and spiral windup of a sheared (tropical cyclone scale) VR wave is $\tau_{v} \equiv 2 \pi r_{v} / v_{v}$, in which $r_{v}$ and $v_{v}$ respectively denote the characteristic radius and azimuthal velocity of the vortex. The time scale for damping of a discrete VR wave is sensitive to the average value of a quantity proportional to the radial gradient of basic-state PV in the critical layer, centered on the surface where $\omega=n \bar{\Omega}(r, z)$ (ibid., see also Schecter 2008). In the preceding surface equation, $\omega$ denotes the angular frequency of the wave, $n=1$ is the azimuthal wavenumber, and $\bar{\Omega} \equiv \bar{v} / r$. The damping time is generally no less than $\tau_{v}$, and is ordinarily much longer when the nondimensional deformation radius $\ell_{d} \equiv N H / \max \left[2 v_{v}, r_{v} f\right]$ substantially exceeds unity. ${ }^{13}$ In the preceding definition of $\ell_{d}, N$ is the Brunt-Väisälä frequency and $H$ is the vertical length scale of the misalignment. Weaker damping with greater $\ell_{d}$ partly results from the attendant lowering of $\omega$ and consequent outward displacement of the critical layer to where the PV gradient is small. Letting $r_{v}=10^{5} \mathrm{~m}$ and $v_{v}=10 \mathrm{~m} \mathrm{~s}^{-1}$ yields $\tau_{v}=17.5 \mathrm{~h}$, which seemingly permits spiral windup to be relevant to an alignment process lasting 1-10 d. However,

\footnotetext{
${ }^{13}$ Weaker damping with greater $\ell_{d}$ is a rule-of-thumb that applies to vortices with relatively smooth (e.g., Gaussian) PV distributions that decay monotonically with increasing $r$. An exception to the rule is known to exist for a special class of Rankine-like vortices (Schecter et al. 2002; Schecter and Montgomery 2003).
} 

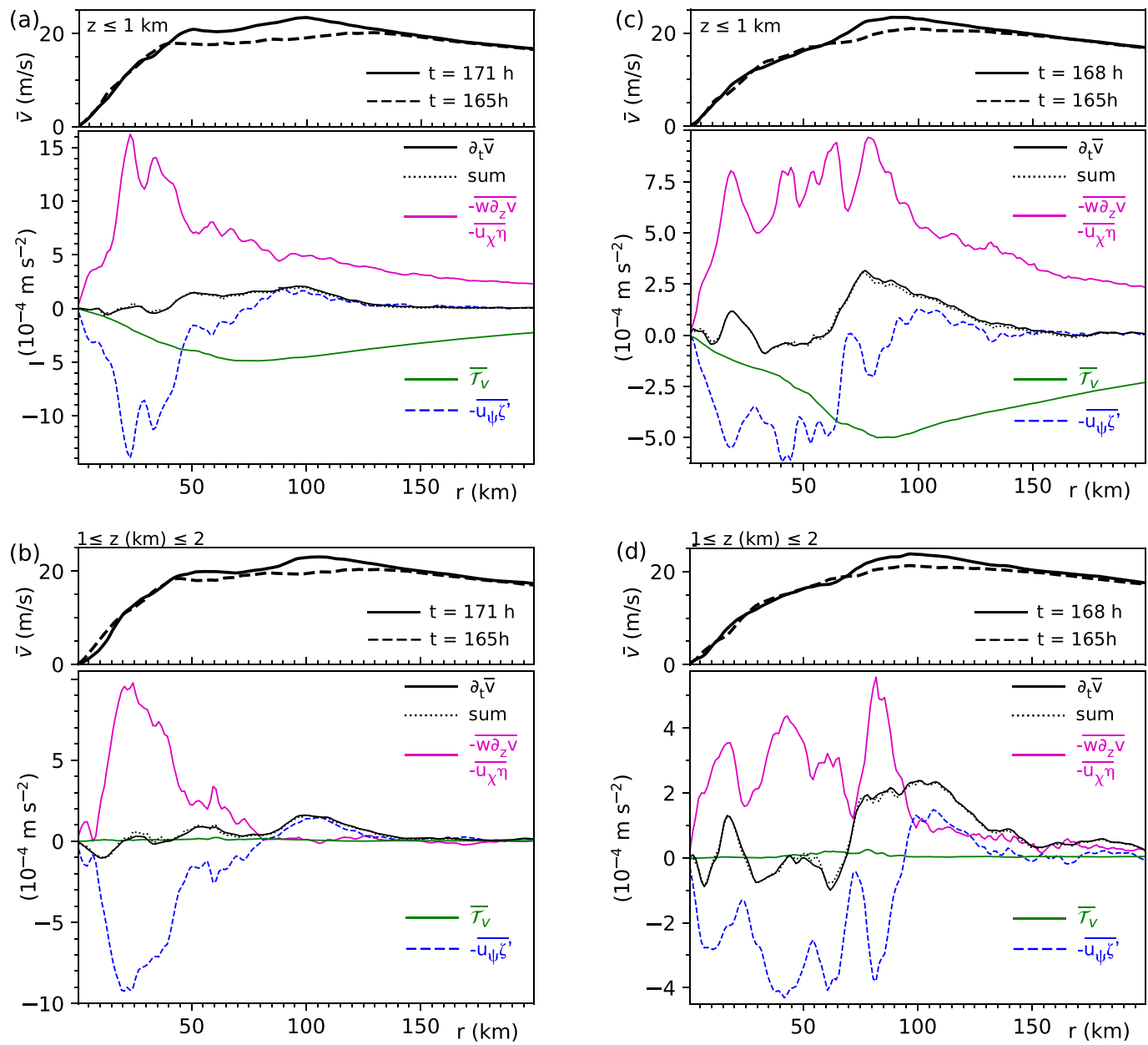

FIG. 23. (a) Change of boundary layer $\bar{v}$ in a fixed coordinate system centered at the end point of the westward trajectory of $\mathbf{x}_{c s}$ in simulation DSPD-X400Z5 during the shaded time interval in Fig. 21a (165 $\leq t \leq 171 \mathrm{~h}$ ). (top) Snapshots of $\bar{v}$ (averaged between $z=0.025$ and $1.01 \mathrm{~km}$ ) at the start (dashed) and end (solid) of the analysis period. (bottom) Tendency of layer-averaged $\bar{v}$ (solid black) and various contributions to that tendency averaged in $t$ over the 6-h analysis period. The contributions from $-\overline{u_{a} \zeta^{\prime}}$ and $-c_{\mathrm{pd}} \overline{\theta_{\rho}^{\prime} \partial_{\varphi} \Pi^{\prime}} / r$ are relatively small $\left(\ll 10^{-4} \mathrm{~m} \mathrm{~s}^{-2}\right)$ and excluded from the plot. The depicted "sum" (dotted black) includes these and all other (magenta, green, and blue) contributions. (b) As in (a), but for layer averages between $z=1.01$ and $2.07 \mathrm{~km}$. (c),(d) As in (a) and (b) but during the period $165 \leq t \leq 168 \mathrm{~h}$ in a fixed coordinate system centered where $\mathbf{x}_{c s}$ resides at $t=168 \mathrm{~h}$.

letting $N=10^{-2} \mathrm{~s}^{-1}, H=5 \times 10^{3} \mathrm{~m}$, and $f=5 \times 10^{-5} \mathrm{~s}^{-1}$ yields $\ell_{d}=2.5$, which marginally exceeds the threshold above which a discrete tilt wave might be expected to linger with very weak damping. Moreover, nonlinear theory suggests that critical layer stirring becomes increasingly ineffective at damping the discrete wave as the tilt magnitude surpasses the length scale of the vortex core (Schecter and Montgomery 2006; Schecter 2008). The preceding considerations leave considerable doubt that classical VR wave mechanisms could drive substantial decay of tilt early on in simulations similar to DSPD-X400Z5.

As time advances, alternative (nonlinear and diabatic) mechanisms can diminish the tilt, cloud coverage may become more diffuse, and the tropical cyclone may intensify to some degree. Cloud-related reduction of $N$ and growth of $v_{v}$ both decrease $\ell_{d}$. Combining this with a smaller perturbation amplitude would increase the frequency $\omega$ of the discrete tilt wave and thereby improve the likelihood of enhanced damping via the wave-critical layer interaction. Accordingly, classical VR wave alignment mechanisms could eventually become relevant. Such potential relevance may often be obscured by concurrent processes, and was not obvious from the basic analysis of section $4 \mathrm{c}$. Further study will be necessary to resolve this issue. Bear in mind that the arrested alignment found in several adiabatic restarts (Fig. 21a) did not disprove the partial relevance of VR 
wave dynamics, partly because the restarts did not incorporate the potentially important cloud-related reduction of $N$.

\section{Conclusions}

This paper has examined the development of tropical cyclones with variable degrees of misalignment in a cloudresolving model set up over a moderately warm ocean on an $f$ plane. The study was distinguished from its predecessors in virtually eliminating ambient vertical wind shear. Our numerical simulations showed that increasing the initial tilt of an incipient tropical cyclone from a negligible value to several hundred kilometers can extend the time scale of hurricane formation from 1 to 10 days. The dramatic slowdown of development in a strongly perturbed system was linked to an extended duration of misalignment resulting from incomplete early decay and subsequent transient growth of the tilt magnitude. Prolonged misalignment coincided with a prolonged period of asymmetric convection peaked far from the rotational center of the surface vortex $\mathbf{x}_{c s}$. Conventional wisdom holds that such a state of affairs generally frustrates the intensification of the maximum tangential wind speed.

To elaborate, the incipient tropical cyclones were found to immediately begin a major realignment phase lasting up to 1 day. The mechanism was examined in detail for a selected simulation starting with an exceptionally large $(367 \mathrm{~km})$ tilt. As in other simulations, $\mathbf{x}_{c s}$ migrated toward an area of vigorous deep cumulus activity in the general direction of the middle-tropospheric rotational center $\mathbf{x}_{c m}$. Moreover, $\mathbf{x}_{c m}$ abruptly jumped to a pronounced vorticity anomaly that formed within the same mesoscale convective system.

Setting the initial tilt magnitude to appreciably exceed the $100-\mathrm{km}$ length scale of the vortex core generally prevented a direct route to alignment, and enabled substantial regrowth of the tilt magnitude over a 1-2 day time scale following its initial decay. For the aforementioned case study, a similar propensity for the restoration of misalignment was found in two dry adiabatic restarts with slightly different initializations and computational configurations. Furthermore, a nondivergent 2D fluid model correctly predicted the direction and magnitude of the drift of $\mathbf{x}_{c m}$ away from $\mathbf{x}_{c s}$ that largely accounted for the regrowth of tilt. Analysis (of the moist 3D system) suggested that an anticyclonic component of the nondivergent velocity field $\mathbf{u}_{\psi}$ associated with an external negative vorticity patch nudged the cyclonic vorticity core containing $\mathbf{x}_{c m}$ in a manner that could assist the observed drift. That being said, diabatic processes persisted and affected the evolution of the tilt vector. Deeper analysis revealed that convection and irrotational winds modulated the specific changes of $\mathbf{u}_{\psi}$ that our center-finding algorithm used to determine the motion of $\mathbf{x}_{c m}$. Moreover, the moist system restored the misalignment more slowly and less completely than the dry adiabatic systems.

The final stage of the alignment process lasted several days in tropical cyclones initialized with relatively large tilts. In the simulation selected for detailed examination, diabatic processes were clearly essential to the ultimate reduction of tilt; the removal of moisture at different times arrested or reversed the decay trend. The final stage of alignment seemed to involve intricate mechanics, as illustrated during a late 6-h westward surge of the surface center toward an area of deep convection containing its middle-tropospheric counterpart. The surge coincided with the mixing of subvortices and filaments within the broader surface cyclone, amid convective forcing weighted to the west. The motion of $\mathbf{x}_{c S}$ was linked to a modest amplification of maximal (lower-tropospheric) $\bar{v}$ in a coordinate system centered at the end point of the trajectory. The net-positive influx of vorticity driven by nondivergent winds $\left(-\overline{u_{\psi} \zeta^{\prime}}\right)$ notably provided a significant contribution to the aforementioned spinup.

The extent to which the foregoing dynamics of a misaligned tropical cyclone depends on details of the experimental setup remains to be seen. Sensitivity to variation of the sea surface temperature and the inclusion of a radiation parameterization merits further investigation. Of equal interest would be a study on sensitivity to the basic structure of the vortex prior to the initial misalignment, which is a well-known issue in the related dry adiabatic problem (Jones 2000a,b; Schecter and Montgomery 2003; Reasor et al. 2004).

Overall, the results of our numerical experiments appear to have corroborated inferences drawn from earlier studies (with sustained shear) on how misalignment reorganizes convection and inhibits tropical cyclone development. A robust positive linear correlation was found between the time-averaged tilt of a developing system (tilt $\mathrm{hf}_{\mathrm{hf}}$ ) and the time required for the surface-maximum tangential velocity $v_{m}$ to reach the threshold wind speed of a hurricane. Relatively large values of tilt $\mathrm{h}_{\mathrm{hf}}$ and slower development were associated with enhanced asymmetry (downtilt bias) of the precipitation probability distribution and larger values of the characteristic precipitation radius $r_{p}$ in the surface-vortex-centered coordinate system. The growth of $r_{p}$ with increasing tilt $\mathrm{hf}_{\mathrm{h}}$ coincided with a commensurate growth of the radius $r_{m}$ at which $v_{m}$ is located.

The consequences of tilt-related modifications to tropical cyclone structure on the mean-vortex spinup mechanism were briefly examined in the context of a 
Sawyer-Eliassen (SE) model. The SE-based analysis provided a reasonably accurate decomposition of the early azimuthal velocity tendency in the vicinity of $v_{m}$, in selected systems with low and high degrees of misalignment. Although no single factor completely explained slower intensification in the strongly misaligned system, greater tilt was linked to weaker positive azimuthal velocity forcing near $v_{m}$ by the component of the mean secondary circulation attributed to heating by microphysical cloud processes.

One notable aspect of the present simulation set was minimal system-to-system variation in the growth of kinetic energy contained outside the core of the primary surface circulation over the time scale required for an aligned vortex to mature. In other words, the detrimental effect of tilt on surface kinetic energy growth was confined to the inner region of the surface vortex.

We conclude with a final remark on the relevance of this paper to forecasting. Although a strong correlation was found between tilt $t_{\mathrm{hf}}$ and $\tau_{\mathrm{hf}}$, our results suggest that an instantaneous measurement of the tilt magnitude may not be a good predictor of the time scale for future intensification of $v_{m}$, even in a quiescent environment. Such is evident from the nonmonotonic evolution of the tilt magnitude. Distinct time scales would be found for equivalent magnitudes measured during different growth or decay phases of the misalignment.

Acknowledgments. The authors thank Dr. George Bryan of the National Center for Atmospheric Research (NCAR) for providing the cloud-resolving model (CM1) that was used for the numerical experiments. The authors also thank several anonymous reviewers for their generally constructive comments on the submitted manuscript. This paper was primarily supported by the National Science Foundation under Grant AGS-1743854. Additional support came from the Natural Sciences and Engineering Research Council of Canada, and from Hydro-Quebec through the IRC program. The computational resources required to conduct the simulations were provided by NCAR's Computational and Information Systems Laboratory (doi:10.5065/D6RX99HX), and by Compute Canada.

\section{APPENDIX A}

\section{Vortex States Generated by Artificial Misalignment Forcing}

Figure A1 depicts a number of selected vortices immediately after their misalignments are generated by methods explained in section 2a. Figures A1a and A1b show a vortex that is misaligned using the DSPD method, with $z_{l}=5.25 \mathrm{~km}$ and a target tilt magnitude of $400 \mathrm{~km}$. The top panel shows relative vertical vorticity and relative humidity in a vertical plane parallel to the tilt vector [Eq. (4)] and passing through the surface vortex center. Both fields are 500-km centered averages over the coordinate $y^{\prime}$ that measures horizontal distance perpendicular to the visualization plane. The relative humidity assumes ice (liquid) condensate above (below) the freezing level. The bottom panel shows horizontal slices of lower- and middle-tropospheric vorticity. Note that the misalignment process 1 ) reduces middletropospheric relative humidity over the central and uptilt regions of the surface vortex, and 2) reweights the negative component of the vorticity distribution toward the east in the outer part of the middle-tropospheric circulation. Figures A1c and A1d are similar to Figs. A1a and A1b, but for a DSPD experiment with a target tilt magnitude of $100 \mathrm{~km}$. Here, the aforementioned consequences of the misalignment process are considerably less pronounced.

Figures A1e and A1f show the vortex from ISPD$\mathrm{X} 400 \mathrm{Z} 5$, in which the tilt is generated while convection is active over the first $6 \mathrm{~h}$ of the simulation. The misalignment is moderately smaller than its counterpart in the DSPD experiment with equivalent settings for the target tilt magnitude and $z_{l}$ (Figs. A1a,b). The convection has also generated a dipole-asymmetry within the predominantly cyclonic core of the middle-tropospheric vortex. The intensity of the negative vorticity hole within the core is found to decay with decreasing values of the target tilt magnitude (not shown). Of further note, as in the aforementioned DSPD experiment, the misaligned vortex has diminished negative vorticity in the outer western region of the middle troposphere. Figures A1g and A1h are similar to Figs. A1e and A1f, but for ISPD-X300Z3.

\section{APPENDIX B}

\section{Sensitivity to the Center-Finding Algorithm}

The "vortex center" at a specified altitude is not uniquely defined in tropical cyclone meteorology. One might wonder how changing the definition would affect the description of the dynamics. This appendix aims to remove some of the mystery for the present study without an exhaustive sensitivity test.

Limited testing has shown that reducing $l_{c}$ in the standard center-finding algorithm of section $2 \mathrm{~b}$ ordinarily adds noise to the time series of $\mathbf{x}_{c}$ without changing the main trend over any extended time period of interest. The preceding result seems obvious and unworthy of lengthy 

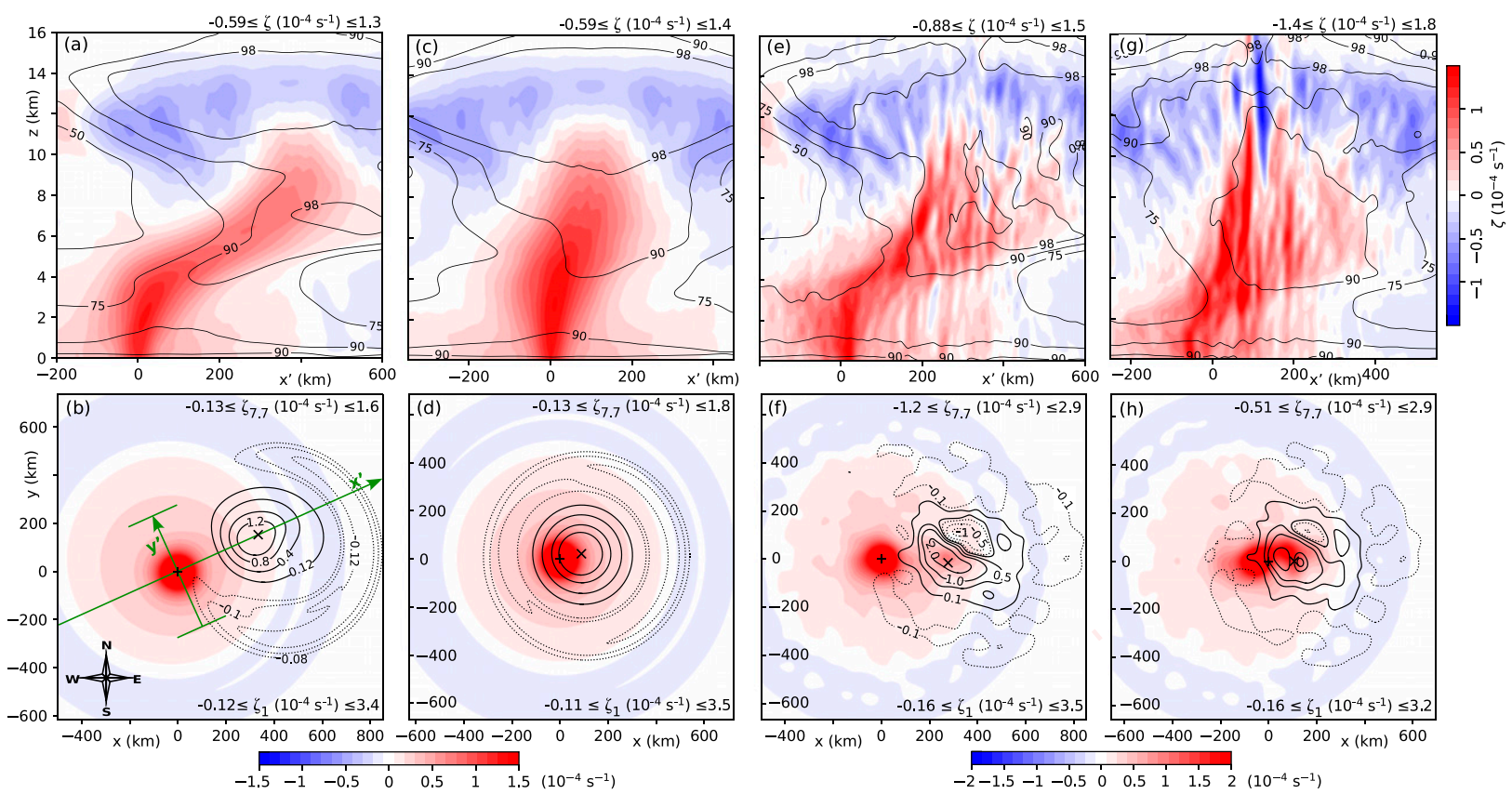

FIG. A1. Vortex states immediately after misalignments are generated $[t=0 \mathrm{~h}(6 \mathrm{~h})$ for DSPD (ISPD) experiments]. (a) Relative vertical vorticity (color) and relative humidity (contours; \%) in a vertical plane parallel to the tilt vector and passing through the surface vortex center in experiment DSPD-X400Z5. (b) Relative vertical vorticity at $z=1.0 \mathrm{~km}$ ( $\zeta_{1}$; color) and $z=7.7 \mathrm{~km}\left(\zeta_{7.7}\right.$; contours). The contour labels are in units of $10^{-4} \mathrm{~s}^{-1}$. The locations of the surface and middle-tropospheric vortex centers $\left(\mathbf{x}_{c s}\right.$ and $\mathbf{x}_{c m}$ defined in section 2b) are marked by the symbols + and $\times$, respectively. The green $x^{\prime}\left(y^{\prime}\right)$ axis runs parallel (perpendicular) to the tilt vector, which points from + to $\times$. The fields in (a) are averaged over the 500-km distance between the depicted end-bars of the $y^{\prime}$ axis; in addition, they are smoothed using a Gaussian kernel with standard deviations of $6.25 \mathrm{~km}$ in $x^{\prime}$ and $0.15 \mathrm{~km}$ in $z$. The fields in (b) are smoothed using a Gaussian kernel with a standard deviation of $30 \mathrm{~km}$ in both $x$ and $y$. (c),(d) As in (a) and (b), but for experiment DSPD-X100Z5. (e),(f) As in (a) and (b), but for experiment ISPD-X400Z5. (g),(h) As in (a) and (b), but for experiment ISPD-X300Z3. The color bar to the right of (g) applies to all panels in the top row. The contour levels in (d) are as in (b), whereas the contour levels in (h) are as in (f). The origin of the coordinate system in each plot corresponds to the center of the surface vortex.

discussion. Instead, let us consider the following alternative definition of a cyclonic vortex center in an arbitrary layer:

$$
\tilde{\mathbf{x}}_{c} \equiv \frac{\int_{A} \mathbf{x} \zeta \Theta\left(\zeta-\zeta_{c}\right) d A}{\int_{A} \zeta \Theta\left(\zeta-\zeta_{c}\right) d A},
$$

in which the area $A$ of integration covers the entire horizontal domain of the simulation, $\mathbf{x}$ is the horizontal coordinate vector, $\zeta$ is the $z$-averaged relative vertical vorticity distribution of the pertinent layer smoothed as in section $2 \mathrm{~b}$ with $l_{c}=23.75 \mathrm{~km}$, and $\Theta\left(\zeta-\zeta_{c}\right)=1(0)$ for $\zeta>\zeta_{c}\left(\zeta<\zeta_{c}\right)$. The cut-off vorticity is given by

$$
\zeta_{c} \equiv \epsilon_{\zeta} \max (\zeta)
$$

in which $0<\epsilon_{\zeta}<1$.

Figure B1a compares the tilt magnitude in DSPDX400Z5 computed with our standard method to that obtained by replacing $\mathbf{x}_{c s / c m}$ with $\tilde{\mathbf{x}}_{c s / c m}$ in Eq. (4). The modification of the time series is modest for $\epsilon_{\zeta}=0.1$ but nontrivial for $\epsilon_{\zeta}=0.02$. The greatest discrepancies begin when vorticity anomalies generated by convection in the middle troposphere create a sufficiently strong meso- $\beta$-scale rotational flow structure far away from the prior consensus vortex center $(t \approx 10,75 \mathrm{~h})$. At such times, the middle-tropospheric flow cannot be said to consist of a single vortex.

Figures B1b-d show snapshots of the streamlines and magnitude of the middle-tropospheric nondivergent velocity field after an early event when the single vortex model of the middle-tropospheric flow becomes questionable. The standard location of $\mathbf{x}_{c m}$ (found as in section $2 \mathrm{~b}$ ) and the locations of $\tilde{\mathbf{x}}_{c m}$ obtained with $\epsilon_{\zeta}=0.1$ and 0.02 are indicated on each snapshot; so too are the surface centers. The first snapshot (Fig. B1b) reveals substantial differences between the standard computation of $\mathbf{x}_{c m}$ and its alternatives. At this time, two noteworthy mesoscale vortices exist. One is the large northeastern cyclone originating from the initial conditions, and the other is a small convectively generated 

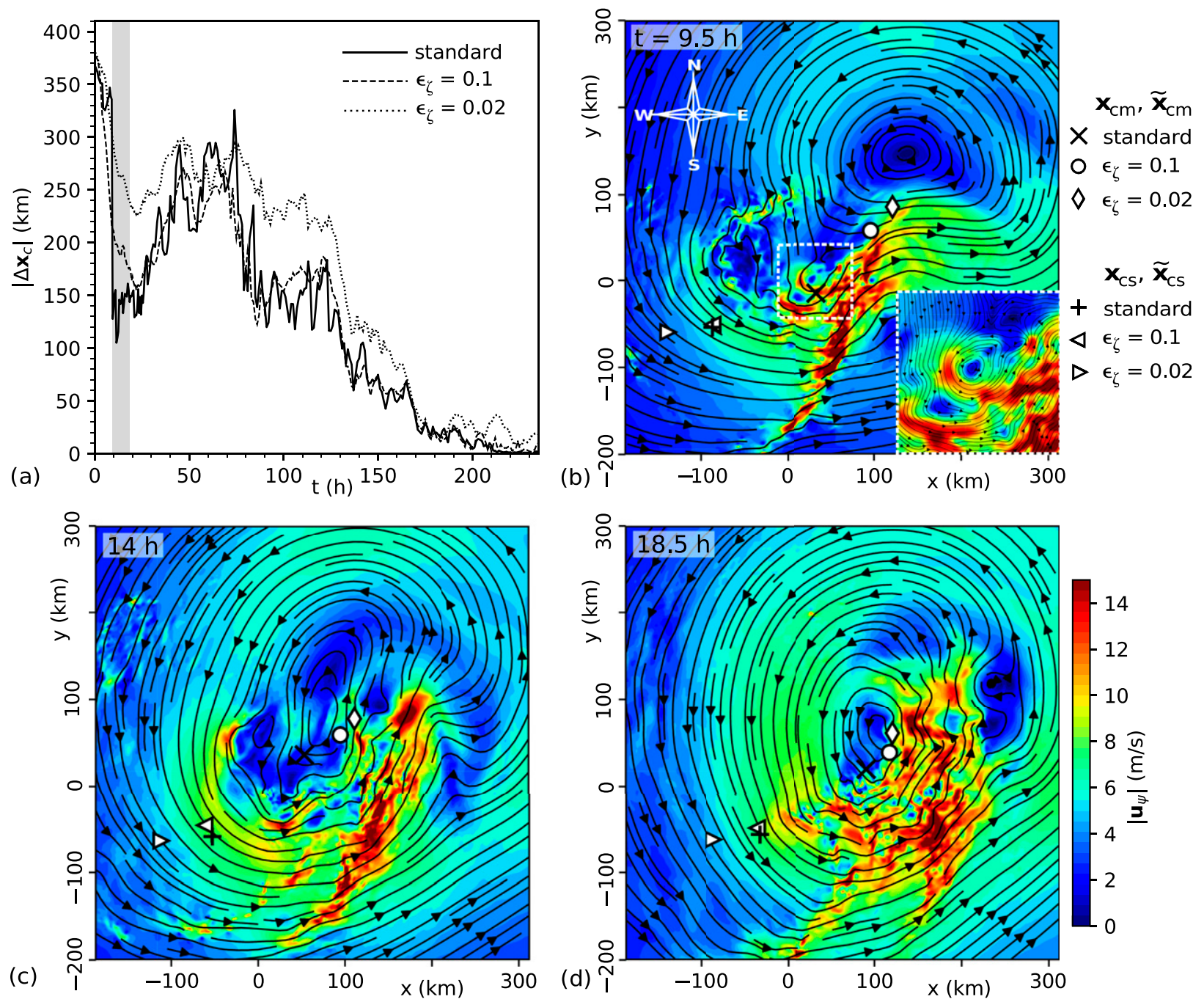

FIG. B1. (a) Time series of the tilt magnitude in experiment DSPD-X400Z5 computed with standard vortex centers (see section $2 \mathrm{~b}$ ) and alternative vortex centers defined by Eqs. (B1) and (B2) with $\epsilon_{\zeta}=0.1$ and $\epsilon_{\zeta}=0.02$. (b)-(d) Snapshots of DSPD-X400Z5 during the shaded time interval in (a), in the domain-centered ES reference frame. (b) Computed vortex centers (see legend) superimposed on the streamlines and magnitude of the nondivergent velocity field $\mathbf{u}_{\psi}$ at $t=9.5 \mathrm{~h}$, vertically averaged over the middle-tropospheric layer defined by $7.34 \leq z \leq 8.13 \mathrm{~km}$. The inset magnifies the flow within the dashed white square. (c),(d) As in (b), but for $t=14$ and $18.5 \mathrm{~h}$, respectively.

vortex (whose details are shown by the inset) coupled to an intensified stream of southwesterly winds. ${ }^{\text {B1 }}$ The standard center-finding algorithm locates a point near the smaller but stronger vortex. The $\zeta$-weighted averages of $\mathbf{x}$ [Eq. (B1)] return points between the cores of the two vortices. Lowering $\epsilon_{\zeta}$ from 0.1 to 0.02 shifts $\tilde{\mathbf{x}}_{c m}$ toward the core of the larger but weaker cyclone. As the two vortical flows coalesce (Figs. B1c,d), all three measurements of the nominal middle-tropospheric vortex center converge. We note in

\footnotetext{
${ }^{\mathrm{B} 1}$ See Fig. $17 \mathrm{c}$ for a depiction of the relative vertical vorticity distribution in the vicinity of the small vortex, which has a substantial negative component east of the positive core.
}

passing that the relative positions of the surface centers remain fairly steady over the depicted time frame, as each drifts eastward toward the area of enhanced cumulus activity.

\section{APPENDIX C}

\section{Helmholtz Velocity Decomposition and Nonlinear Balance}

The Helmholtz decomposition of the horizontal velocity field may be written as

$$
\mathbf{u}=\mathbf{u}_{\chi}+\mathbf{u}_{\psi}+\mathbf{u}_{a}
$$


The irrotational component is given by $\mathbf{u}_{\chi} \equiv \nabla_{h} \chi$, in which $\nabla_{h}$ is the horizontal gradient operator and $\nabla_{h}^{2} \chi=\nabla_{h} \cdot \mathbf{u} \equiv \sigma$. The nondivergent component is given by $\mathbf{u}_{\psi} \equiv \hat{\mathbf{z}} \times \nabla_{h} \psi$, in which $\nabla_{h}^{2} \psi=\hat{\mathbf{z}} \cdot\left(\nabla_{h} \times \mathbf{u}\right) \equiv \zeta$. The additional component $\mathbf{u}_{a}$ is both irrotational and nondivergent. For this paper, the Helmholtz decomposition is generally constructed by solving the Poisson equation for $\psi$ with periodic boundary conditions, equating $\mathbf{u}_{a}$ to $\langle\mathbf{u}\rangle_{x y}$, and extracting $\mathbf{u}_{\chi}$ from Eq. (C1). Note that $\mathbf{u}_{a}$ is identical to the ambient velocity field defined in the main text, which may vary with the motion of the reference frame. Throughout this appendix, the subscript $a$ will denote the $z$-dependent horizontal domain average (ambient part) of a fluid variable, whereas the prefix $\delta$ will denote a perturbation from that average.

One element of nonlinear balance (McWilliams 1985) is that $\mathbf{u}_{\psi}$ dominates $\mathbf{u}_{\chi}$, such that

$$
\mathbf{u} \approx \mathbf{u}_{\psi}+\mathbf{u}_{a} .
$$

A second element of nonlinear balance is that the tendency equation for the divergence of the horizontal flow $\sigma$ reduces to a diagnostic equation for the pressure perturbation. The approximation amounts to neglecting all terms involving $\sigma, \mathbf{u}_{\chi}, w$ and parameterized turbulence in the aforementioned tendency equation. Such neglect yields

$$
\nabla_{h}^{2} \delta \Pi \approx \frac{2}{c_{p d} \theta_{v a}}\left[\partial_{x x} \psi \partial_{y y} \psi-\left(\partial_{x y} \psi\right)^{2}\right]+\frac{f \zeta}{c_{p d} \theta_{v a}},
$$

in which $x$ and $y$ are orthogonal Cartesian coordinates, $\Pi \equiv\left(p / p_{0}\right)^{R_{d} / c_{p d}}$ is the Exner function, $R_{d}\left(c_{p d}\right)$ is the gas constant (isobaric specific heat) of dry air, and $p_{0} \equiv 10^{5} \mathrm{~Pa}$. Here and elsewhere, $\partial_{\alpha} \equiv \partial / \partial \alpha$ and $\partial_{\alpha \beta} \equiv \partial^{2} / \partial \alpha \partial \beta$ denote the first and second order partial derivatives with respect to the generic variables $\alpha$ and $\beta$. Equation (C3) is readily solved for $\delta \Pi$ (given $\zeta$ and $\psi$ at arbitrary $z$ ) under the assumptions of doubly periodic boundary conditions and zero horizontal mean. Note that Eq. (C3) uses the approximation $\rho^{-1} \nabla_{h} \delta p \approx c_{p d} \theta_{v a} \nabla_{h} \delta \Pi$, in which $\rho$ is density. Note further that Eq. (C3) reduces to gradient wind balance (cyclostrophic balance if $f=0$ ) when $\zeta$ is axisymmetric.

A third element of nonlinear balance is the complementary hydrostatic equation, here approximated by

$$
\delta \theta_{v} \approx \frac{c_{p d} \theta_{v a}^{2}}{g} \partial_{z} \delta \Pi
$$

in which $g$ is the gravitational acceleration, $\delta \theta_{v} \equiv \theta_{v}-$ $\theta_{v a}$, and $\delta \Pi$ is the solution to Eq. (C3). In this paper, the system is said to be in a state of nonlinear balance if approximations $(\mathrm{C} 2)-(\mathrm{C} 4)$ are satisfied.

\section{APPENDIX D}

\section{Sawyer-Eliassen-Based Analysis of Vortex Spinup}

Taking the $\varphi$ average of the azimuthal velocity equation in the SVC reference frame (or a stationary reference frame) yields

$$
\partial_{t} \bar{v}=-\bar{u} \bar{\eta}-\bar{w} \partial_{z} \bar{v}+\overline{\mathcal{E}}_{v}+\overline{\mathcal{T}}_{v},
$$

in which

$$
\overline{\mathcal{E}}_{v} \equiv-\overline{u^{\prime} \zeta^{\prime}}-\overline{w^{\prime} \partial_{z} v^{\prime}}-\overline{\theta^{\prime} \partial_{\varphi} \tilde{\Pi}^{\prime}} / r
$$

is the mean acceleration associated with resolved "eddy" transport (plus a second-order pressure torque), $\mathcal{T}_{v}$ is an acceleration associated with unresolved turbulence (plus Rayleigh damping), $\eta \equiv \zeta+f$ is the absolute vertical vorticity, and $\tilde{\Pi} \equiv c_{p d} \Pi$. Note that the pressure gradient term in Eq. (D1b) assumes that the density potential temperature $\theta_{\rho}$ is adequately approximated by $\theta$.

The complementary $\varphi$-averaged thermodynamic equation may be written for the variable $\kappa \equiv \theta^{-1}$ as follows:

$$
\partial_{t} \bar{\kappa}=-\bar{u} \partial_{r} \bar{\kappa}-\bar{w} \partial_{z} \bar{\kappa}+\overline{\mathcal{E}}_{\kappa}-\overline{\kappa^{2} \dot{\theta}},
$$

in which

$$
\overline{\mathcal{E}}_{\kappa} \equiv-\overline{u^{\prime} \partial_{r} \kappa^{\prime}}-\overline{v^{\prime} \partial_{\varphi} \kappa^{\prime}} / r-\overline{w^{\prime} \partial_{z} \kappa^{\prime}}
$$

is the mean tendency associated with eddy transport and $\dot{\theta}$ includes all sources contributing to the material derivative of $\theta$. It is convenient to let

$$
\dot{\theta}=\dot{\theta}_{\mathrm{mp}}+\mathcal{T}_{\theta},
$$

in which $\dot{\theta}_{\mathrm{mp}}$ accounts for microphysical cloud processes, whereas $\mathcal{T}_{\theta}$ accounts for unresolved turbulent transport, dissipative heating, Rayleigh damping, and a minor tendency linked to 3D velocity-divergence in moist air (e.g., Bryan and Fritsch 2002). Recall that radiative transfer is neglected in this study.

The Sawyer-Eliassen (SE) equation provides approximations for $\bar{u}$ and $\bar{w}$ under the key assumption that they act to maintain thermal wind balance in the mean vortex as it responds to the slow forcing of $\bar{v}$ and $\bar{\kappa}$. Herein, we express thermal wind balance by

$$
\partial_{z}(C \bar{\kappa})=-g \partial_{r} \bar{\kappa}
$$

in which $C \equiv \bar{v}^{2} / r+f \bar{v}$. It is also assumed that the continuity equation can be reduced to $\partial_{r}(\bar{\rho} \bar{u} r)+\partial_{z}(\bar{\rho} \bar{w} r)=0$, such that $\bar{\rho} \bar{u} r=-\partial_{z} \Psi$ and $\bar{\rho} \bar{w} r=\partial_{r} \Psi$. Taking the partial 
time derivative of Eq. (D3) and substituting Eqs. (D1a) and (D2a) into the result yields the following SE equation:

$$
\mathcal{L}[\Psi]=F_{\mathrm{mp}}+F_{e}+F_{\mathcal{T}} .
$$

The linear operator on the left-hand side of Eq. (D4) is defined such that

$$
\mathcal{L}[\Psi] \equiv \partial_{z}\left(\frac{I \partial_{z} \Psi+B \partial_{r} \Psi}{\bar{\rho} r}\right)+\partial_{r}\left(\frac{S \partial_{r} \Psi+B \partial_{z} \Psi}{\bar{\rho} r}\right),
$$

in which the baroclinity, static stability, and modified inertial stability parameters are respectively given by

$$
\begin{aligned}
B & \equiv-\partial_{z}(C \bar{\kappa}), \\
S & \equiv-g \partial_{z} \bar{\kappa}, \\
I & \equiv \bar{\kappa} \bar{\eta} \bar{\xi}+B C / g .
\end{aligned}
$$

In addition, the forcing functions satisfy

$$
\begin{aligned}
F_{\mathrm{mp}} & \equiv \partial_{z}\left(C \overline{\kappa^{2} \dot{\theta}_{\mathrm{mp}}}\right)+g \partial_{r}\left(\overline{\kappa^{2} \dot{\theta}_{\mathrm{mp}}}\right), \\
F_{e} & \equiv-\partial_{z}\left(C \overline{\mathcal{E}}_{\kappa}\right)-g \partial_{r} \overline{\mathcal{E}}_{\kappa}-\partial_{z}\left(\bar{\kappa} \bar{\xi} \overline{\mathcal{E}}_{v}\right), \\
F_{\mathcal{T}} & \equiv \partial_{z}\left(C \overline{\kappa^{2} \mathcal{T}_{\theta}}\right)+g \partial_{r}\left(\overline{\kappa^{2} \mathcal{T}_{\theta}}\right)-\partial_{z}\left(\bar{\kappa} \bar{\xi} \overline{\mathcal{T}}_{v}\right) .
\end{aligned}
$$

Note that $\bar{\xi} \equiv 2 \bar{v} / r+f$.

The linearity of the SE equation conveniently permits a decomposition of the secondary circulation into contributions from each forcing function, such that $\Psi=\Psi_{\mathrm{mp}}+\Psi_{e}+\Psi_{\mathcal{T}}$ and likewise for $\bar{u}$ and $\bar{w}$. With such a decomposition, we may rewrite the velocity tendency [Eq. (D1a)] as follows

$$
\partial_{t} \bar{v}=\mathcal{A}_{\mathrm{mp}}+\mathcal{A}_{e}+\mathcal{A}_{\mathcal{T}}+\overline{\mathcal{E}}_{v}+\overline{\mathcal{T}}_{v}+\epsilon,
$$

in which $\mathcal{A}_{\alpha} \equiv-\bar{u}_{\alpha} \bar{\eta}-\bar{w}_{\alpha} \partial_{z} \bar{v}$ for all $\alpha \in\{\mathrm{mp}, e, \mathcal{T}\}$. The correction $\epsilon$ is included to acknowledge the inexact nature of the SE solution for $\bar{u}$ and $\bar{w}$. Note (for example) that $\mathcal{A}_{\mathrm{mp}}$ does not represent the entire effect of diabatic cloud processes on vortex spinup, since removing clouds would change all other accelerations. Examination of $\mathcal{A}_{\mathrm{mp}}$ simply reveals how an imaginary secondary circulation that acts to maintain thermal wind balance under the heating associated with cloud processes would spin up (or spin down) the vortex in the absence of eddy forcing and turbulent transport.

The main text considers the integral of each term in Eq. (D8) over a relatively short time period $(\delta t=6 \mathrm{~h})$ using data stored every $10 \mathrm{~min}$. The time integrals of $\partial_{t} \bar{v}, \overline{\mathcal{E}}_{v}$, and $\overline{\mathcal{T}}_{v}$ are determined by straightforward quadrature. The time integral of each term associated with the mean-flow advection of angular momentum is taken to be $\delta t \tilde{\mathcal{A}}_{\alpha}$, in which $\tilde{\mathcal{A}}_{\alpha}$ is an approximation of the time-average of $\mathcal{A}_{\alpha}$. In this approximation, $\bar{u}_{\alpha}$ and $\bar{w}_{\alpha}$ are obtained from solutions of the SE equation with time averages of the simulation data used for all azimuthally averaged variables. Similar time averages are used for $\bar{\eta}$ and $\partial_{z} \bar{v}$ appearing in the definition of $\mathcal{A}_{\alpha}$. Note that using simulation data for both $\bar{v}$ and $\bar{\kappa}$ is not precisely consistent with the assumption of thermal wind balance, but is deemed adequate for the purpose of our analysis.

The solution for each component of the SE equation is obtained with the boundary conditions $\Psi_{\alpha}=0$ at $r=0$, $r=r_{o}, z=0$, and $z=z_{t}$, in which $r_{o}=919 \mathrm{~km}$ and $z_{t}=$ $29.2 \mathrm{~km}$. Small (and even negative) static stability in some regions of the boundary layer may cause violation of the following ellipticity condition:

$$
\begin{gathered}
I S-B^{2}>0 \\
\rightarrow \quad \bar{\eta} \bar{\xi} S>-\bar{\xi} \partial_{z} \bar{v} B .
\end{gathered}
$$

Such violation may introduce a solvability problem. No such complication occurred for the analysis of DSPDX400Z5. For the analysis of the control experiment, ellipticity was restored in regions of violation below $z=$ $400 \mathrm{~m}$ (where it so happens that $\bar{\eta} \bar{\xi}>0$ and $\partial_{z} \bar{v} B / \bar{\eta}<0$ ) by the transformation $S \rightarrow \max \left[S,-(1+\nu) \partial_{z} \bar{v} B / \bar{\eta}\right]$, in which $\nu$ is a small positive parameter. The solution presented in the main text corresponds to $\nu=0.001$ but hardly differs from its counterpart with $\nu=1.0$. Despite such insensitivity, one should bear in mind that the necessity of an ad hoc regularization leaves the solution (at least in the boundary layer) somewhat questionable.

\section{APPENDIX E}

\section{Middle-tropospheric $\zeta$ Budget during Stage 1 of DSPD-X400Z5}

The Eulerian time derivative of the relative vertical vorticity distribution $(\zeta)$ is given by

$$
\begin{aligned}
\partial_{t} \zeta= & -\mathbf{u} \cdot \nabla_{h} \zeta-w \partial_{z} \zeta-\eta \nabla_{h} \cdot \mathbf{u}+\zeta_{h} \cdot \nabla_{h} w \\
& -c_{p d} \hat{\mathbf{z}} \cdot\left(\nabla_{h} \theta_{\rho} \times \nabla_{h} \Pi\right)+\hat{\mathbf{z}} \cdot\left(\nabla_{h} \times \mathbf{T}_{h}\right),
\end{aligned}
$$

in which $\zeta_{h}$ is the horizontal vorticity vector, $\mathbf{T}_{h}$ is the horizontal velocity tendency associated with parameterized turbulence, and all other symbols were introduced earlier. From left to right, the first and second terms on the right-hand side of Eq. (E1) correspond to the horizontal and vertical advection of $\zeta$. The third and fourth terms are associated with vortex-tube stretching and tilting. The fifth and sixth terms are associated with baroclinic and frictional vorticity generation. The horizontal advection and stretching terms are commonly combined into a single term equaling $-\nabla_{h} \cdot \eta \mathbf{u}$. 

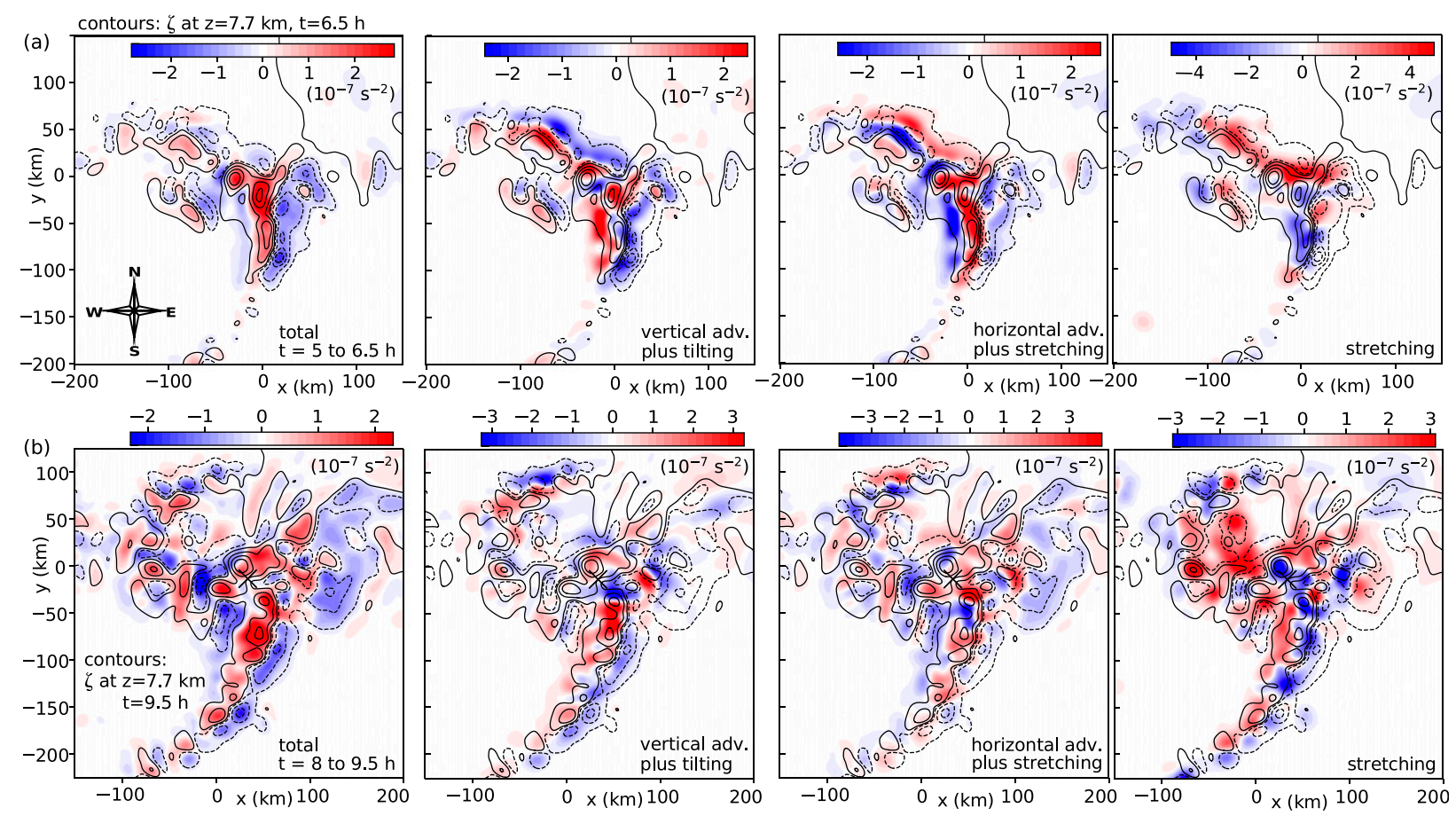

FIG. E1. (a) Analysis of $\partial_{t} \zeta$ in the middle troposphere during the period $5 \leq t \leq 6.5 \mathrm{~h}$ in experiment DSPD-X400Z5. Colors show time averages of (left to right) the sum of all terms on the right-hand side of Eq. (E1), the sum of the vertical advection and tilting terms, the sum of the horizontal advection and stretching terms, and the stretching term alone. The contours show a snapshot of $\zeta$ at the end of the averaging period. The positive (solid) and negative (dashed) contour levels are $\pm[0.05,0.25,0.5,0.75] \zeta_{\max }$, in which $\zeta_{\max }=1.21 \times 10^{-3} \mathrm{~s}^{-1}$ is the maximum magnitude of $\zeta$. All depicted fields are taken at $z=7.7 \mathrm{~km}$ and smoothed with a Gaussian kernel whose standard deviation parameter is $6.25 \mathrm{~km}$ in both horizontal dimensions. (b) As in (a), but during the period $8 \leq t \leq 9.5 \mathrm{~h}$ and with $\zeta_{\max }=1.55 \times 10^{-3} \mathrm{~s}{ }^{-1}$; the location of $\mathbf{x}_{c m}$ at $t=9.5 \mathrm{~h}$ is marked with an $\times$.

Similarly, the vertical advection and tilting terms are commonly combined into a single term equaling $\nabla_{h} \cdot w \hat{\mathbf{z}} \times \partial_{z} \mathbf{u}$. For the analyses considered herein, the baroclinic and frictional terms are characteristically one or more orders of magnitude smaller than all other terms on both sides of Eq. (E1), and are thus considered negligible.

Figure E1 depicts the $\zeta$ budget in the middle troposphere prior to the sudden jump of $\mathbf{x}_{c m}$ toward a region of vigorous cumulus activity during stage 1 of experiment DSPD-X400Z5 (see Fig. 17). Figure E1a (E1b) corresponds to the time interval between hours 5 and 6.5 ( 8 and 9.5) of the simulation. The far-left panel shows the sum of all terms contributing to the right-hand side of Eq. (E1) averaged over the 1.5-h analysis period. The panels to the right show similar time averages of specific contributions. Contours of $\zeta$ at the end of the analysis period (henceforth $\zeta_{e}$ ) are superimposed on each plot. The analysis is conducted in the domain-centered ES reference frame.

One can readily see that the sum of all tendency terms is well-correlated with $\zeta_{e}$ in the displayed vicinity of vigorous convection. Moreover, the "forcing" of $\zeta$ by the combination of vertical advection and tilting tends to have the same sign as $\zeta_{e}$; rarely does the opposite sign occur where $\zeta_{e}$ is substantial. The combination of horizontal advection and stretching partly reinforces and partly opposes vertical advection and tilting. The sign of the stretching term alone does not consistently match that of its combination with horizontal advection or that of all terms summed together. Note that all displayed tendencies have substantial positive and negative parts (see Haynes and McIntyre 1987). Considering the partially negative forcing of $\zeta$ associated with convection, along with the initial eastern crescent of outer negative vorticity (Fig. A1b), it is unsurprising to find a prominent anticyclonic vorticity patch east of the cyclonic core of the middle-tropospheric circulation at the start of stage 2 of the simulation (see section $4 b$ ).

\section{APPENDIX F}

\section{Helmholtz-Based Decomposition of $\partial_{t} \bar{v}$}

In section 4 , the acceleration of $\bar{v}$ is examined from the viewpoint of the following equation: 


$$
\partial_{t} \bar{v}=-\overline{u_{\chi} \eta}-\overline{u_{\psi} \zeta^{\prime}}-\overline{u_{a} \zeta^{\prime}}-\overline{w \partial_{z} v}-c_{p d} \overline{\theta_{\rho}^{\prime} \partial_{\varphi} \Pi^{\prime}} / r+\overline{\mathcal{T}}_{v}
$$

in which all velocities are computed in an ES reference frame. As in Schecter (2017), the influx of absolute vorticity $(-\overline{u \eta})$ has been decomposed into parts driven by the irrotational, nondivergent and ambient components of the radial velocity field. Each component is obtained as explained in appendix C. Note that because $\bar{u}_{\psi}=\bar{u}_{a}=0$, the radial influx of absolute vorticity driven by either $u_{\psi}$ or $u_{a}$ involves only a nominal eddy-contribution. For simplicity, we have refrained from the common practice of splitting $\overline{u_{\chi} \eta}$ and $\overline{w \partial_{z} v}$ into contributions from the mean flow and eddies. The time averages of Eq. (F1) in Figs. 20 and 23 are obtained from simulation data sampled every $3 \mathrm{~min}$.

\section{REFERENCES}

Bryan, G. H., and J. M. Fritsch, 2002: A benchmark simulation for moist nonhydrostatic numerical models. Mon. Wea. Rev., 130, 2917-2928, https://doi.org/10.1175/1520-0493(2002)130<2917: ABSFMN $>2.0 . \mathrm{CO} ; 2$.

Bui, H. H., R. K. Smith, M. T. Montgomery, and J. Peng, 2009: Balanced and unbalanced aspects of tropical-cyclone intensification. Quart. J. Roy. Meteor. Soc., 135, 1715-1731, https://doi.org/ 10.1002/qj.502.

Chen, X., Y. Wang, J. Fang, and M. Xue, 2018: A numerical study on rapid intensification of Typhoon Vicente (2012) in the South China Sea. Part II: Roles of inner-core processes. J. Atmos. Sci., 75, 235-255, https://doi.org/10.1175/JAS-D-17-0129.1.

Davis, C. A., and L. F. Bosart, 2006: The formation of hurricane Humberto (2001): The importance of extra-tropical precursors. Quart. J. Roy. Meteor. Soc., 132, 2055-2085, https:// doi.org/10.1256/qj.05.42.

— , and D. A. Ahijevych, 2012: Mesoscale structural evolution of three tropical weather systems observed during PREDICT. J. Atmos. Sci., 69, 1284-1305, https://doi.org/10.1175/JAS-D-11-0225.1.

$\longrightarrow$, and - 2013: Thermodynamic environments of deep convection in Atlantic tropical disturbances. J. Atmos. Sci., 70, 1912-1928, https://doi.org/10.1175/JAS-D-12-0278.1.

DeMaria, M., J. A. Knaff, and B. H. Connell, 2001: A tropical cyclone genesis parameter for the tropical Atlantic. Wea. Forecasting, 16, 219-233, https://doi.org/10.1175/1520-0434(2001) 016<0219:ATCGPF $>2.0$. CO;2.

Donelan, M. A., B. K. Haus, N. Reul, W. J. Plant, M. Stiassnie, H. C. Graber, O. B. Brown, and E. S. Saltzman, 2004: On the limiting aerodynamic roughness of the ocean in very strong winds. Geophys. Res. Lett., 31, L18306, https://doi.org/10.1029/ 2004GL019460.

Drennan, W. M., J. A. Zhang, J. R. French, C. McCormick, and P. G. Black, 2007: Turbulent fluxes in the hurricane boundary layer. Part II: Latent heat flux. J. Atmos. Sci., 64, 1103-1115, https://doi.org/10.1175/JAS3889.1.

Dunion, J. P., 2011: Rewriting the climatology of the tropical North Atlantic and Caribbean Sea atmosphere. J. Climate, 24, 893 908, https://doi.org/10.1175/2010JCLI3496.1.

Fairall, C. W., E. F. Bradley, J. E. Hare, A. A. Grachev, and J. B. Edson, 2003: Bulk parameterization of air-sea fluxes:
Updates and verification for the COARE algorithm. J. Climate, 16, 571-591, https://doi.org/10.1175/1520-0442(2003)016<0571: BPOASF $>2.0 . C O ; 2$.

Finocchio, P. M., S. J. Majumdar, D. S. Nolan, and M. Iskandarani, 2016: Idealized tropical cyclone responses to the height and depth of environmental vertical wind shear. Mon. Wea. Rev., 144, 2155-2175, https://doi.org/10.1175/MWR-D-15-0320.1.

Ge, X., T. Li, and M. Peng, 2013: Effects of vertical shears and midlevel dry air on tropical cyclone developments. J. Atmos. Sci., 70, 3859-3875, https://doi.org/10.1175/JAS-D-13-066.1.

Gent, P. R., and J. C. McWilliams, 1986: The instability of barotropic circular vortices. Geophys. Astrophys. Fluid Dyn., 35, 209-233, https://doi.org/10.1080/03091928608245893.

Gray, W. M., 1968: Global view of the origins of tropical cyclones. Mon. Wea. Rev., 96, 669-700, https://doi.org/10.1175/1520-0493(1968) 096<0669:GVOTOO>2.0.CO;2.

Haynes, P. H., and M. E. McIntyre, 1987: On the evolution of vorticity and potential vorticity in the presence of diabatic heating and frictional or other forces. J. Atmos. Sci., 44 828-841, https://doi.org/10.1175/1520-0469(1987)044<0828: OTEOVA $>2.0 . \mathrm{CO} ; 2$.

Jones, S. C., 2000a: The evolution of vortices in vertical shear. II: Large-scale asymmetries. Quart. J. Roy. Meteor. Soc., 126, 3137-3159, https://doi.org/10.1002/qj.49712657008.

_ 2000b: The evolution of vortices in vertical shear. III: Baroclinic vortices. Quart. J. Roy. Meteor. Soc., 126, 3161-3185, https://doi.org/10.1002/qj.49712657009.

Kaplan, J., M. DeMaria, and J. A. Knaff, 2010: A revised tropical cyclone rapid intensification index for the Atlantic and eastern North Pacific basins. Wea. Forecasting, 25, 220-241, https:// doi.org/10.1175/2009WAF2222280.1.

Komaromi, W. A., 2013: An investigation of composite dropsonde profiles for developing and nondeveloping tropical waves during the 2010 PREDICT field campaign. J. Atmos. Sci., 70, 542-558, https://doi.org/10.1175/JAS-D-12-052.1.

Leonard, A., 1980: Vortex methods for flow simulation. J. Comput. Phys., 37, 289-335, https://doi.org/10.1016/ 0021-9991(80)90040-6.

McBride, J. L., and R. M. Zehr, 1981: Observational analysis of tropical cyclone formation. Part II: Comparison of nondeveloping versus developing systems. J. Atmos. Sci., 38, 1132-1151, https://doi.org/10.1175/1520-0469(1981)038<1132: OAOTCF $>2.0 . \mathrm{CO} ; 2$.

McWilliams, J. C., 1985: A uniformly valid model spanning the regimes of geostrophic and isotropic, stratified turbulence: Balanced turbulence. J. Atmos. Sci., 42, 1773-1774, https://doi.org/ 10.1175/1520-0469(1985)042<1773:AUVMST>2.0.CO;2.

Molinari, J., D. Vollaro, and K. L. Corbosiero, 2004: Tropical cyclone formation in a sheared environment: A case study. J. Atmos. Sci., 61, 2493-2509, https://doi.org/10.1175/ JAS3291.1.

Montgomery, M. T., and R. K. Smith, 2014: Paradigms for tropical cyclone intensification. Aust. Meteor. Oceanogr. J., 64, 37-66.

Morrison, H., J. A. Curry, and V. I. Khvorostyanov, 2005: A new double-moment microphysics parameterization for application in cloud and climate models. Part I: Description. J. Atmos. Sci., 62, 1665-1677, https://doi.org/10.1175/JAS3446.1.

—, G. Thompson, and V. Tatarskii, 2009: Impact of cloud microphysics on the development of trailing stratiform precipitation in a simulated squall line: Comparison of one-and two-moment schemes. Mon. Wea. Rev., 137, 991-1007, https:// doi.org/10.1175/2008MWR2556.1. 
Musgrave, K. D., C. A. Davis, and M. T. Montgomery, 2008: Numerical simulations of the formation of Hurricane Gabrielle (2001). Mon. Wea. Rev., 136, 3151-3167, https:// doi.org/10.1175/2007MWR2110.1.

Nguyen, L. T., and J. Molinari, 2015: Simulation of the downshear reformation of a tropical cyclone. J. Atmos. Sci., 72, 45294551, https://doi.org/10.1175/JAS-D-15-0036.1.

_, R. F. Rogers, and P. D. Reasor, 2017: Thermodynamic and kinematic influences on precipitation symmetry in sheared tropical cyclones: Bertha and Cristobal (2014). Mon. Wea. Rev., 145, 4423-4446, https://doi.org/10.1175/MWR-D-17-0073.1.

Nolan, D. S., and M. G. McGauley, 2012: Tropical cyclogenesis in wind shear: Climatological relationships and physical processes. Cyclones: Formation, Triggers, and Control, K. Oouchi and H. Fudeyasu, Eds., Nova Science Pub. Inc., 1-35.

Onderlinde, M. J., and D. S. Nolan, 2016: Tropical cyclonerelative environmental helicity and the pathways to intensification in shear. J. Atmos. Sci., 73, 869-890, https:// doi.org/10.1175/JAS-D-15-0261.1.

Polvani, L. M., 1991: Two-layer geostrophic vortex dynamics. Part 2: Alignment and two-layer V-states. J. Fluid Mech., 225, 241270, https://doi.org/10.1017/S0022112091002045.

Rappin, E. D., and D. S. Nolan, 2012: The effect of vertical shear orientation on tropical cyclogenesis. Quart. J. Roy. Meteor. Soc., 138, 1035-1054, https://doi.org/10.1002/qj.977.

Reasor, P. D., and M. T. Montgomery, 2001: Three-dimensional alignment and corotation of weak, TC-like vortices via linear vortex Rossby waves. J. Atmos. Sci., 58, 2306-2330, https://doi.org/ 10.1175/1520-0469(2001)058<2306:TDAACO >2.0.CO;2.

- and - 2015: Evaluation of a heuristic model for tropical cyclone resilience. J. Atmos. Sci., 72, 1765-1782, https:// doi.org/10.1175/JAS-D-14-0318.1.

,-- and L. D. Grasso, 2004: A new look at the problem of tropical cyclones in shear flow: Vortex resiliency. J. Atmos. Sci., 61, 3-22, https://doi.org/10.1175/1520-0469(2004)061<0003: ANLATP $>2.0 . \mathrm{CO} ; 2$.

Rios-Berrios, R., C. A. Davis, and R. D. Torn, 2018: A hypothesis for the intensification of tropical cyclones under moderate vertical wind shear. J. Atmos. Sci., 75, 4149-4173, https:// doi.org/10.1175/JAS-D-18-0070.1.

Ryglicki, D. R., and R. E. Hart, 2015: An investigation of centerfinding techniques for tropical cyclones in mesoscale models. J. Appl. Meteor. Climatol., 54, 825-846, https://doi.org/10.1175/ JAMC-D-14-0106.1.

Schecter, D. A., 2008: The spontaneous imbalance of an atmospheric vortex at high Rossby number. J. Atmos. Sci., 65, 24982521, https://doi.org/10.1175/2007JAS2490.1.

, 2013: Relationships between convective asymmetry, imbalance and intensity in numerically simulated tropical cyclones. Tellus $A, \mathbf{6 5}, 20168$, https://doi.org/10.3402/tellusa.v65i0.20168.

- 2015: Response of a simulated hurricane to misalignment forcing compared to predictions of a simple theory. J. Atmos. Sci., 72, 1235-1260, https://doi.org/10.1175/JAS-D-14-0149.1.
- 2016: Development and nondevelopment of binary mesoscale vortices into tropical cyclones in idealized numerical experiments. J. Atmos. Sci., 73, 1223-1254, https://doi.org/ 10.1175/JAS-D-15-0028.1

2017: A computational study on the nature of meso- $\beta$ scale vortex coalescence in a tropical atmosphere. J. Adv. Model. Earth Syst., 9, 1366-1398, https://doi.org/10.1002/2016MS000777. , and M. T. Montgomery, 2003: On the symmetrization rate of an intense geophysical vortex. Dyn. Atmos. Oceans, 37, 55-88, https://doi.org/10.1016/S0377-0265(03)00015-0.

, and - 2006: Conditions that inhibit the spontaneous radiation of spiral inertia-gravity waves from an intense mesoscale cyclone. J. Atmos. Sci., 63, 435-456, https://doi.org/ 10.1175/JAS3641.1.

— and - 2007: Waves in a cloudy vortex. J. Atmos. Sci., 64, 314-337, https://doi.org/10.1175/JAS3849.1.

and P. D. Reasor, 2002: A theory for the vertical alignment of a quasigeostrophic vortex. J. Atmos. Sci., 59, 150-168, https:// doi.org/10.1175/1520-0469(2002)059<0150:ATFTVA >2.0.CO;2.

Schubert, W. H., and J. J. Hack, 1982: Inertial stability and tropical cyclone development. J. Atmos. Sci., 39, 1687-1697, https:// doi.org/10.1175/1520-0469(1982)039<1687:ISATCD>2.0.CO;2.

Shapiro, L. J., and H. E. Willoughby, 1982: The response of balanced hurricanes to local sources of heat and momentum. J. Atmos. Sci., 39, 378-394, https://doi.org/10.1175/1520-0469(1982)039<0378: TROBHT $>2.0 . \mathrm{CO} ; 2$.

Smith, R. K., and M. T. Montgomery, 2012: Observations of the convective environment in developing and non-developing tropical disturbances. Quart. J. Roy. Meteor. Soc., 138, 17211739, https://doi.org/10.1002/qj.1910.

- - — a and H. Zhu, 2005: Buoyancy in tropical cyclones and other rapidly rotating atmospheric vortices. Dyn. Atmos. Oceans, 40, 189-208, https://doi.org/10.1016/j.dynatmoce.2005.03.003.

Tang, B., and K. Emanuel, 2012: A ventilation index for tropical cyclones. Bull. Amer. Meteor. Soc., 93, 1901-1912, https:// doi.org/10.1175/BAMS-D-11-00165.1.

Tao, D., and F. Zhang, 2014: Effect of environmental shear, seasurface temperature, and ambient moisture on the formation and predictability of tropical cyclones: An ensemble-mean perspective. J. Adv. Model. Earth Syst., 6, 384-404, https:// doi.org/10.1002/2014MS000314.

Tory, K. J., N. E. Davidson, M. T. Montgomery, and J. D. Kepert, 2007: Prediction and diagnosis of tropical cyclone formation in an NWP system. Part III: Diagnosis of developing and nondeveloping storms. J. Atmos. Sci., 64, 3195-3213, https:// doi.org/10.1175/JAS4023.1.

Viera, F., 1995: On the alignment and axisymmetrization of a vertically tilted geostrophic vortex. J. Fluid Mech., 289, 29-50, https://doi.org/10.1017/S0022112095001224.

Zhang, J. A., P. G. Black, J. R. French, and W. M. Drennan, 2008: First direct measurements of enthalpy flux in the hurricane boundary layer: The CBLAST results. Geophys. Res. Lett., 35, L14813, https://doi.org/10.1029/2008GL034374. 